\title{
Exosomes Act as Molecular Vehicles \\ Contributing to Cellular Cholesterol Efflux
}

\author{
Doctoral Thesis \\ In partial fulfillment of the requirements for the degree \\ "Doctor rerum naturalium (Dr. rer. nat.)" \\ in the Molecular Medicine Study Program \\ at the Georg-August-University Göttingen
}

submitted by

Katrin Strauss

born in

Sondershausen, Germany

Göttingen, December 14, 2010 
Members of the Thesis Committee:

Supervisor

Prof. Dr. Thomas A. Bayer

Department for Psychiatry, Georg-August-University Göttingen

Second member of the thesis committee

Prof. Dr. Mikael Simons

Max Planck Institute of Experimental Medicine, Göttingen

Department of Neurology, Georg-August-University Göttingen

Third member of the thesis committee

Prof. Dr. Fred S. Wouters

Department of Neurophysiology, University Medicine Göttingen

CMPB, DFG Research Center Molecular Physiology of the Brain, Göttingen

Date of Disputation: 


\section{Affidavit}

Here I declare that my doctoral thesis entitled "Exosomes act as molecular vehicles contributing to cellular cholesterol efflux" has been written independently with no other sources and aids than quoted.

Katrin Strauss

Göttingen, December 2010 


\section{Related publication}

Katrin Strauss, Cornelia Goebel, Heiko Runz, Wiebke Moöbius, Sievert Weiss, Ivo Feussner, Mikael Simons, and Anja Schneider. (2010) Exosome Secretion Ameliorates Lysosomal Storage of Cholesterol in Niemann-Pick Type C Disease. The Journal of Biological Chemistry; 285(34): 26279-26288 


\section{Contents}

Contents $\quad$ v

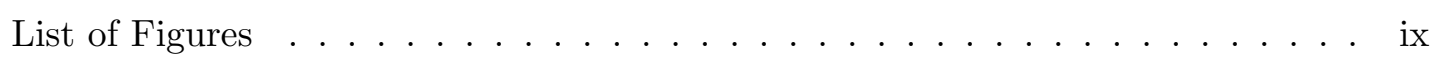

List of Tables . . . . . . . . . . . . . . . . . . . . xi

Abbreviations $\quad$ xii

Acknowledgments $\quad$ xv

Abstract $\quad$ xvii

1 Introduction $\quad 1$

1.1 Exosomes . . . . . . . . . . . . . . . . . . . . . 1

1.1.1 Morphology and composition . . . . . . . . . . . . . 3

1.1.2 Biogenesis of exosomes . . . . . . . . . . . . . . . . 5 5

1.1.3 Release of exosomes . . . . . . . . . . . . . . . . 8

1.1.4 Function of exosomes . . . . . . . . . . . . . . . . 9

1.2 The flotillin proteins $\ldots \ldots \ldots \ldots \ldots \ldots$

$1.2 .1 \quad$ Protein structure . . . . . . . . . . . . . . . . . . 12

1.2 .2 Protein functions . . . . . . . . . . . . . . . . . . . 15

1.3 Cholesterol in health and disease . . . . . . . . . . . . . . . . 16

1.3.1 Mechanisms of cholesterol synthesis and uptake . . . . . . . . . 18

1.3.2 Intracellular cholesterol pathways . . . . . . . . . . . . . . . . 19

1.3.3 Mechanisms of cholesterol efflux . . . . . . . . . . . . . . 21

1.3.4 Role of cholesterol in disease . . . . . . . . . . . . . . . . 22

1.4 Niemann-Pick type $\mathrm{C}$ disease . . . . . . . . . . . . . . . . 23

1.4.1 Structure and function of the NPC1 and NPC2 proteins . . . . . . 25

1.4.2 Molecular mechanisms of NPC pathology . . . . . . . . . . . . 27

1.5 Aims of the study . . . . . . . . . . . . . . . . 30 
2 Materials and Methods $\quad 32$

2.1 Materials . . . . . . . . . . . . . . . . . . . . 32

2.1.1 Chemicals and consumables . . . . . . . . . . . . . 32

2.1.2 Cell lines and primary cells . . . . . . . . . . . . . . . 32

2.1.2.1 Cell lines . . . . . . . . . . . . . . . . . . 32

2.1.2.2 Primary cells . . . . . . . . . . . . . 33

2.1 .3 Antibodies . . . . . . . . . . . . . . . . . 33

2.1.4 Nucleotide constructs . . . . . . . . . . . . . . . . . . 33

2.1.4.1 Plasmids . . . . . . . . . . . . . . . 33

2.1.4.2 SiRNA constructs . . . . . . . . . . . . . 34

2.1.5 Modifiers of cellular cholesterol homeostasis . . . . . . . . . . . . 34

2.1.6 Buffers and solutions . . . . . . . . . . . . . . . . . . 35

2.1.6.1 Phosphate buffered saline $(\mathrm{PBS}) \ldots \ldots \ldots$

2.1.6.2 HEPES/sucrose stock solution . . . . . . . . . . . 35

2.1.6.3 CHAPS lysis buffer . . . . . . . . . . . . . . 36

2.1.6.4 Protein loading buffer . . . . . . . . . . . . 36

2.1 .7 Media and sera . . . . . . . . . . . . . . . 36

2.1.7.1 Commercial media . . . . . . . . . . . . . . 36

2.1 .7 .2 SATO medium . . . . . . . . . . . . 37

2.1.7.3 General growth medium . . . . . . . . . . . . . 37

2.1 .8 Commercial kits . . . . . . . . . . . . . . . . . . 37

2.1 .9 Software . . . . . . . . . . . . . . . . . 38

2.2 Methods . . . . . . . . . . . . . . . . . . . 38

2.2 .1 Molecular biology . . . . . . . . . . . . . . . 38

2.2.1.1 Site-directed mutagenesis . . . . . . . . . . . 38

2.2.1.2 Transformation of E. coli . . . . . . . . . . . . . . 39

2.2.1.3 Plasmid DNA isolation from E. coli . . . . . . . . . . . 39

2.2.1.4 Determination of DNA concentration . . . . . . . . 39

$2.2 .2 \quad$ Cell culture . . . . . . . . . . . . . . . . . . . . . . . . . . . 39

2.2.2.1 Cryoconservation of cells . . . . . . . . . . 40

2.2.2.2 Growth and maintenance of cells . . . . . . . . . . . . 40

2.2.2.3 Modification of cellular cholesterol . . . . . . . . . . . . 41

2.2.2.4 Collection of exosomes . . . . . . . . . . . . 43

2.2.2.5 Transfection of plasmids . . . . . . . . . . . . 43 
2.2.2.6 RNA interference . . . . . . . . . . . . . 43

2.2 .3 Exosome purification . . . . . . . . . . . . . . . . . 44

2.2.3.1 Exosome preparation . . . . . . . . . . . . . 44

2.2.3.2 Sucrose gradient ultracentrifugation . . . . . . . . . . . 44

2.2.4 Protein and lipid biochemistry . . . . . . . . . . . . . . 45

2.2.4.1 Determination of protein concentration . . . . . . . 45

2.2 .4 .2 SDS-PAGE . . . . . . . . . . . . . . 46

2.2.4.3 Western blotting . . . . . . . . . . . . . 47

2.2.4.4 Cholesterol extraction and gas chromatography . . . . . . 47

2.2 .5 Immunocytochemistry . . . . . . . . . . . . . . . . . . 48

2.2.5.1 Immunofluorescence staining . . . . . . . . . . . . 48

2.2.5.2 Lysotracker staining . . . . . . . . . . . . . . . . 49

2.2.5.3 Filipin staining . . . . . . . . . . . . . . . . 49

2.2 .6 Microscopy . . . . . . . . . . . . . . . . . . 5 50

2.2.6.1 Confocal microscopy . . . . . . . . . . . . . . 50

2.2.6.2 Electron microscopy . . . . . . . . . . . . . . 50

2.2.7 Image processing and statistical analysis . . . . . . . . . . . . 50

2.2.7.1 Quantification of subcellular flotillin-2 distribution . . . . 50

2.2.7.2 Quantification of exosome secretion . . . . . . . . . 51

2.2.7.3 Statistical analysis . . . . . . . . . . . . . . 51

3 Results $\quad 53$

3.1 Cellular cholesterol levels regulate formation and release of exosomes . . . . 53

3.1.1 Cholesterol regulates the cellular distribution of flotillin-2 . . . . . 53

3.1.1.1 Subcellular localization of flotillin-2 . . . . . . . . . 54

3.1.1.2 Cholesterol depletion redistributes flotillin-2 to the plasma membrane . . . . . . . . . . . . . . 54

3.1.1.3 Cholesterol loading accumulates flotillin-2 in endosomal pools ....................... 56

3.1.1.4 Intracellular cholesterol accumulation directs flotillin-2 to endosomal pools . . . . . . . . . . . . . . 5 58

3.1.2 Exosome release depends on cholesterol . . . . . . . . . . . . 61

3.1.2.1 Purification of exosomes . . . . . . . . . . . . . 61

3.1.2.2 Cellular cholesterol levels influence exosome release . . . . 63 
3.1.2.3 The delayed exosome pathway depends on cholesterol . . . 67

3.2 Flotillin-2 interacts with cholesterol and regulates its exosomal release . . . 69

3.2.1 Two cholesterol recognition/interaction amino acid consensus (CRAC) sequences mediate subcellular distribution and exosomal release of flotillin-2 . . . . . . . . . . . . . . . . . 69

3.2.2 Flotillin-2 down-regulation impairs exosomal cholesterol release . . . 71

3.3 Exosomal cholesterol release is up-regulated in Niemann-Pick type C disease 74

3.3.1 Exosome release is enhanced in NPC . . . . . . . . . . . . . 74

3.3.2 Cholesterol release via exosomes is increased in NPC . . . . . . . . 80

3.3.3 Exosome release can be reduced by transfection of wild-type NPC1 in NPC1-mutants . . . . . . . . . . . . . . . . . . . . 80

\section{Discussion}

4.1 Flotillin-2 is distributed in response to cholesterol levels . . . . . . . . . . 85

4.1.1 Flotillin-2 distribution depends on cholesterol . . . . . . . . . . 85

4.1.2 Flotillin's CRAC domains facilitate its interaction with cholesterol . 87

4.2 Flotillin-2 recruits cholesterol to exosomes . . . . . . . . . . . . . . 88

4.2.1 Exosomal release of cholesterol depends on flotillin- $2 \ldots$. . . . . . 88

4.2.2 Flotillin-dependent endocytosis . . . . . . . . . . . . . . . . . . 89

4.2.3 Parallels of flotillin- and caveolin-mediated cholesterol mobility . . . 90

4.3 Exosomes may contribute to cellular cholesterol homeostasis . . . . . . . . . 92

4.3.1 Exosome secretion is adjusted to cholesterol levels . . . . . . . . . 92

4.3.2 The immediate and the delayed exosome pathway are differently influenced by cholesterol . . . . . . . . . . . . . . . . . . 93

4.4 Exosomal cholesterol release in NPC . . . . . . . . . . . . . . . . 95

4.4.1 Cholesterol release via exosomes is increased in NPC . . . . . . . . . 95

4.4.2 Exocytosis of accumulating material in disease . . . . . . . . . . 97

4.4.3 How does exosomal cholesterol release affect the NPC phenotype? . 98

4.4 .4 Fate of exosomes . . . . . . . . . . . . . . . . . 98

4.4.5 Exosomes as biomarkers for NPC . . . . . . . . . . . . . 99

5 Summary and Conclusions $\quad 102$

$\begin{array}{lr}\text { Bibliography } & 104\end{array}$

$\begin{array}{ll}\text { Curriculum Vitae } & 134\end{array}$ 


\section{List of Figures}

1.1 Generation and release of exosomes . . . . . . . . . . . . . . 2

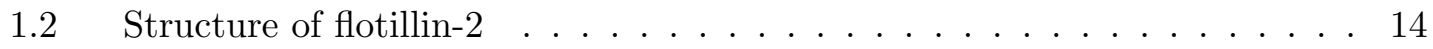

1.3 Synthesis of cholesterol . . . . . . . . . . . . . . . . . 17

1.4 Cellular cholesterol pathways . . . . . . . . . . . . . . . . . 20

1.5 Cholesterol accumulation in NPC . . . . . . . . . . . . . . . 24

2.1 Quantification of subcellular flotillin-2 distribution . . . . . . . . . . 51

3.1 Flotillin-2 is localized to endosomes and lysosomes . . . . . . . . . . 55

3.2 Cholesterol depletion redistributes flotillin-2 to the plasma membrane . . 57

3.3 Cholesterol loading accumulates flotillin-2 in endosomal pools . . . . . . . 59

3.4 Intracellular trapping of cholesterol facilitates flotillin-2 accumulation in endosomal pools . . . . . . . . . . . . . . . . . . 60 60

3.5 Exosomes can be purified by a sequence of centrifugation steps . . . . . . 62

3.6 Sucrose gradient ultracentrifugation of the exosome pellet . . . . . . . . 63

$3.7 \quad$ Cholesterol depletion decreases exosome release . . . . . . . . . . . . . . . 64

3.8 Sucrose gradient ultracentrifugation of exosome pellets derived from cholesterol loaded cells . . . . . . . . . . . . . . . . . . 65

3.9 Loading of free cholesterol increases exosome release . . . . . . . . . 66

3.10 Cholesterol affects the delayed exosome pathway but not immediate vesicle shedding from the plasma membrane . . . . . . . . . . . . 68

3.11 CRAC domains are required for intracellular vesicular localization of flotillin-

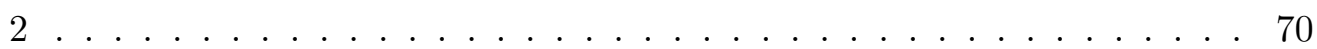

3.12 CRAC domains are necessary for the exosomal release of flotillin-2 . . . 71

3.13 Flotillin-2 is required for the exsosomal release of cholesterol . . . . . . . . 73

3.14 Cholesterol accumulation in late endosomes/lysosomes but not in the ER increases exosome release . . . . . . . . . . . . . . . 75

3.15 Loss of functional NPC1 increases exosome release . . . . . . . . . . . 77 
3.16 Exosome release is increased in human skin fibroblasts derived from an NPC patient . . . . . . . . . . . . . . . . . . . . . . 79

3.17 Exosomal cholesterol release is increased in $\mathrm{NPC}$. . . . . . . . . . . 81

3.18 Transfection rates of wild-type and mutant NPC1 are similar . . . . . . . 83

3.19 Transfection of NPC1-mutant CHO cells with wild-type NPC1 restores normal exosome release . . . . . . . . . . . . . . . . . . . . 84 84 


\section{List of Tables}

$2.1 \quad$ Cell lines . . . . . . . . . . . . . . . . . . . . . . . . . 32

2.2 Primary antibodies . . . . . . . . . . . . . . . 33

2.3 Plasmids . . . . . . . . . . . . . . . . . . . . . 34

$2.4 \quad$ siRNA constructs . . . . . . . . . . . . . . . . . . 34

2.5 Cholesterol modifiers . . . . . . . . . . . . . . . . . . 35

2.6 Commercial media and solutions . . . . . . . . . . . . 36

2.7 Commercial kits . . . . . . . . . . . . . . . . . . . . . . 38

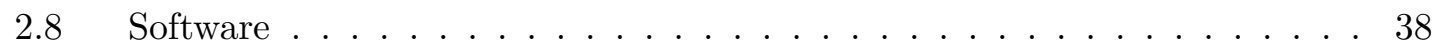

2.9 Sucrose density gradient . . . . . . . . . . . . . . . . . 45 


\section{Abbreviations}

aa

$\mathrm{A} \beta$

ABCA1

$\mathrm{AD}$

ACAT

apo

APS

CAP

CE

$\mathrm{CEH}$

CHAPS

CHO cells

CNS

CRAC

DC

DNA

EBV

ECR

EDTA

EGF

ESCRT

ER

FCS

$\mathrm{g}$

GFP

GPI-anchored protein
Amino acid

Amyloid beta peptide

ATP-binding cassette transporter 1

Alzheimer's disease

Acyl-coenzyme A:cholesterol acyltransferase

Apolipoportein

Ammonium persulfate

c-Cbl associated protein

Cholesteryl ester

Cholesteryl ester hydrolase

3-[3-(Cholamidopropyl)dimethylammonio]-1-proanesulfonate

Chinese hamster ovary cells

Central nervous system

Cholesterol recognition/interaction amino acid consensus sequences

Dendritic cell

Deoxyribonucleic acid

Epstein-Barr virus

Evolutionary constrained region

Ethylenediaminetetraacetic acid

Epidermal grotwh factor

Endosomal sorting complex required for transprort

Endoplasmic reticulum

Fetal calf serum

Gramm

Green fluorescent protein

Glycosylphosphatidylinositol-anchored protein 
GTPase

h

HDL

HEPES

HIV

HMG-CoA

HS

HRP

ILV

$\mathrm{kDa}$

L

LAMP-1

LBPA

LDL

LMP2A

$\mathrm{m} \beta \mathrm{CD}$

MFGE8

MHC

$\min$

MLD

MVB

NB-DNJ

NPC

$\mathrm{NSF}$

NTD

PAGE

PBS

PBR

PFA

PI3 kinase

$\mathrm{PIP}_{3}$

PLL

PLP

PrP
Guanine triphosphatase

$\operatorname{Hour}(\mathrm{s})$

High-density lipoprotein

4-(2-hydroxyethyl)-1-piperazineethanesulfonic acid

Human immunodeficiency virus

3-hydroxy-3-methylglutaryl-CoA

Horse serum

Horse radish peroxidase

Intraluminal vesicle

Kilodalton

Liter

Lysosomal-associated membrane protein 1

Lysobisphophatidic acid

Low-density lipoprotein

Latent membrane protein $2 \mathrm{~A}$

Methyl- $\beta$-cyclodextrin

Milk-fat-globule EGF-factor VIII

Major histocompatibility complex

Minute(s)

Metachromatic leukodystrophy

Multivesicular body

N-butyldeoxynojirimycin

Niemann-Pick type $\mathrm{C}$ disease

N-ethylmaleimide-sensitive factor

$\mathrm{N}$-terminal domain

Polyacrylamide gel electrophoresis

Phosphate buffered saline

Peripheral benzodiazepine receptor

Paraformaldehyde

Phosphoinositide 3 kinase

Phosphatidylinositol(3,4,5)-trisphosphate

Poly-L-lysine

Proteolipid protein

Prion protein 
PS

PNS

Rab

RNA

$\mathrm{s}$

SCAP

SC-B1

SDS

SEM

SH3 domain

SNAP

SNARE

SoHo domain

SPFH

SREBP

SSD

stAR

START protein

STAM

TEMED

TSG-101

U18666A

YFP
Phosphatidylserine

Peripheral nervous system

Ras like protein in brain

Ribonucleic acid

Second(s)

SREBP-cleavage-activating protein

Scavenger receptor B1

Sodium dodecyl sulphate

Standard error of the mean

SRC homology 3 domain

Synaptosomal-associated protein

Soluble NSF attachment protein receptor

Sorbin homology domain

stomatin/prohibitin/flotilllin/hflK/C

Sterol-regulatory element-binding protein

Sterol-sensing domain

Steroidogenesis acute regulatory protein

StAR-related lipid transfer protein

Signal-transducing adaptor molecule

N'N'N'-tetramethylethylene diamine

tumor-susceptibility gene 101

3- $\beta$-(2-diethylaminoethoxy) androst-5-en-17-one

Yellow fluorescent protein 


\section{Acknowledgments}

I deeply thank my direct supervisor Anja Schneider for her advice and guidance that encouraged me throughout the successes and setbacks of my thesis. I am grateful for sharing her scientific and technical expertise with me, which invaluably broadened my scientific horizon and shaped up my ideas and research. I gratefully acknowledge the members of my thesis committee, Mikael Simons, Thomas A. Bayer and Fred S. Wouters, for their constructive and fruitful comments on this thesis.

It is a pleasure to express my gratitude to the collaborators, who crucially participated in making my thesis a success. I would like to thank Cornelia Goebel and Ivo Feußner from the Department for Plant Biochemistry, Göttingen for the gas chromatographic analysis of my samples. Furthermore, I would like to acknowledge Wiebke Möbius from the MaxPlanck-Institute of Experimental Medicine, Göttingen for performing electron microscopy on exosomes and Heiko Runz form the Department of Human Genetics, Heidelberg for providing me with human NPC1 fibroblasts and microscopical data on them. I am much indebted to Sievert Weiß who started this project together with Anja Schneider and performed some of the initial experiments that set the basis for my thesis.

To conduct my work and present the project on international conferences would not have been possible without the administrative and financial support of the University Medicine Göttingen and the DFG Research Center Molecular Physiology of the Brain (CMPB). I further thank Thomas A. Bayer for welcoming me in the Neurodegeneration in Alzheimer's Disease Marie Curie Ph.D. Graduate Program (NEURAD) as an associated member, thereby giving me the opportunity to exchange scientific ideas and experiences during the NEURAD International Summer Schools. 
Many thanks go in particular to my colleagues and friends in $A G$ Simons for their patient help in teaching me new techniques, for the stimulating scientific discussions and most of all for the pleasant working atmosphere, the movie nights and game evenings. These are: Giselheid Schulz, Kirsten Fladung, Dirk Fitzner, Angelika Kippert, Chieh Hsu, Larisa Yurlova, Holger Budde, Sebastian Richter, Hannes Treiber, Mostafa Bakhti, Natalia Manrique-Hoyos, Aniket Gosh, Shweta Aggarwal, Johannes Zimmermann, Olena Steshenko, Petra Wilken, Nicolas Snaidero, Alexander Korolov, Christina Reetz and Karla Hans. I especially want to express my thanks to Mareike Schnaars and Sebastian Schmitt for creating such a great friendship in the lab and beyond.

I would like to thank Ditte Christensen, Andrea Marcello, Marie Cotel, Indronil and Ariane Chaudhuri and Cathrin Enke, for sharing the joyful moments I experienced in Leuven, Lille, St. Andreasberg and Göttingen.

My special thanks go to my close friends and companions Martin Schloms, Antje Faßhauer, Felix Glahn, Thomas Beer, Daniel Volkmann, Falk Sinning and Michael Ridders for making my life truly rich and valuable. Finally, I want to thank my family, especially my mother, Tanja Strauß, and my father, Reimund Ohm, for their unconditional support, unlimited love and persistent confidence in me. More then once their listening and advice has taken the load off my shoulders and guided my way. I am very proud of being their daughter. 


\section{Abstract}

Exosomes are small vesicles of 50-100 nm diameter that derive from intraluminal vesicles (ILVs) and are secreted into the extracellular environment upon fusion of multiversicular bodies (MVBs) with the plasma membrane. Release of exosomes is reported for a variety of cell types and serves numerous functions including the shuttle of RNA, proteins and lipids for cell-to-cell communication, antigen presentation, and disposal of obsolete or superfluous molecules. Exosomes display a cell type-specific lipid composition, for example those released by B lymphocytes are enriched in cholesterol. Since a tight regulation of cholesterol homeostasis is indispensable for the structural and functional integrity of each cell, we asked whether exosomes contribute to this process by facilitating cholesterol egress.

This study shows that generation and release of exosomes is regulated by cellular cholesterol levels. While exosome release was decreased after methyl- $\beta$-cyclodextrin-mediated cholesterol depletion of oligodendroglial cells, loading of cholesterol led to its increase. It is further demonstrated that flotillin-2, a membrane associated protein enriched in exosomes, is impaired in its exosomal secretion after mutating its putative cholesterol recognition/interaction amino acid consensus (CRAC) sequences. SiRNA-mediated down-regulation of flotillin-2 significantly reduced exosomal cholesterol release, highlighting flotillin's importance for cholesterol trafficking.

Translocation of cholesterol from late endosomes/lysosomes to the ER and Golgi compartments has previously been shown to depend on NPC1 and NPC2 proteins. In NiemannPick type $\mathrm{C}$ disease (NPC), loss-of-function mutations of these proteins result in late endosomal/lysosomal accumulation of unesterified cholesterol and sphingolipids, clinically leading to progressive demyelination, neurodegeneration and eventually to death. Here, it is shown that oligodendroglial cells upregulate their exosomal cholesterol secretion upon induction of NPC-like late endosomal/lysosomal cholesterol trapping either by administration of U18666A or by siRNA-mediated down-regulation of NPC1. A comparable 
upregulation was seen in primary fibroblasts of NPC1 patients and in NPC1-mutant Chinese hamster ovary cells, where transient expression of wild-type NPC1 was able to reverse the phenotype.

These findings indicate that exosomes may serve as molecular vehicles partially bypassing the cholesterol traffic block in NPC1 that causes pathological accumulation of cholesterol in late endosomal/lysosomal compartments. Moreover, they indicate a role for exosome secretion in contributing to the maintenance of cellular cholesterol homeostasis. 


\section{Chapter 1}

\section{Introduction}

\subsection{Exosomes}

A hallmark of all eukaryotic cells is their compartmentalization into complex, membraneenclosed organelles, which fulfill discrete tasks and work in concert to ensure cell viability. To facilitate the communication between these compartments, the cell employs vesicular structures trafficking through the cytosol. These molecular vehicles are not only used to shuttle cargo between intracellular membranes. Cells also release a subpopulation of vesicles into their environment for purposes of cargo degradation or cell-to-cell communication. There is a variety of extracellular vesicles, whose release depends on type and condition of the parent cell. One example are microvesicles, which have a heterogeneous size of $100 \mathrm{~nm}$ - $1 \mu \mathrm{m}$ and bud directly from the plasma membrane (1). In contrast, smaller vesicles with a diameter of approximately $50-100 \mathrm{~nm}$ originate from the endosomal system and are released as exosomes upon membrane fusion (see Figure 1.1) (2).

The existence of exosomes was first documented in 1983 by the group of Rose M. Johnstone. They described a process, in which transferrin receptors are selectively released via small vesicles during the maturation of reticulocytes into erythrocytes (4; 5). A few years later, it was proposed that these 'exosomes' are formed within the same pathway described for the trafficking of transferrin receptors (6). According to the authors, exosomes correspond to the intraluminal vesicles (ILVs) found in multivesicular bodies (MVBs), that are generated by inward budding of endosomes. Upon fusion of MVBs with the plasma membrane, exosomes are released into the extracellular space (7). This pathway was initially assumed to be utilized for the release of obsolete material from cells (6). However, intensive research on exosome biology over the past 20 years led to insights emphasizing their general importance for a variety of cell biological processes. Exosomes have been reported 


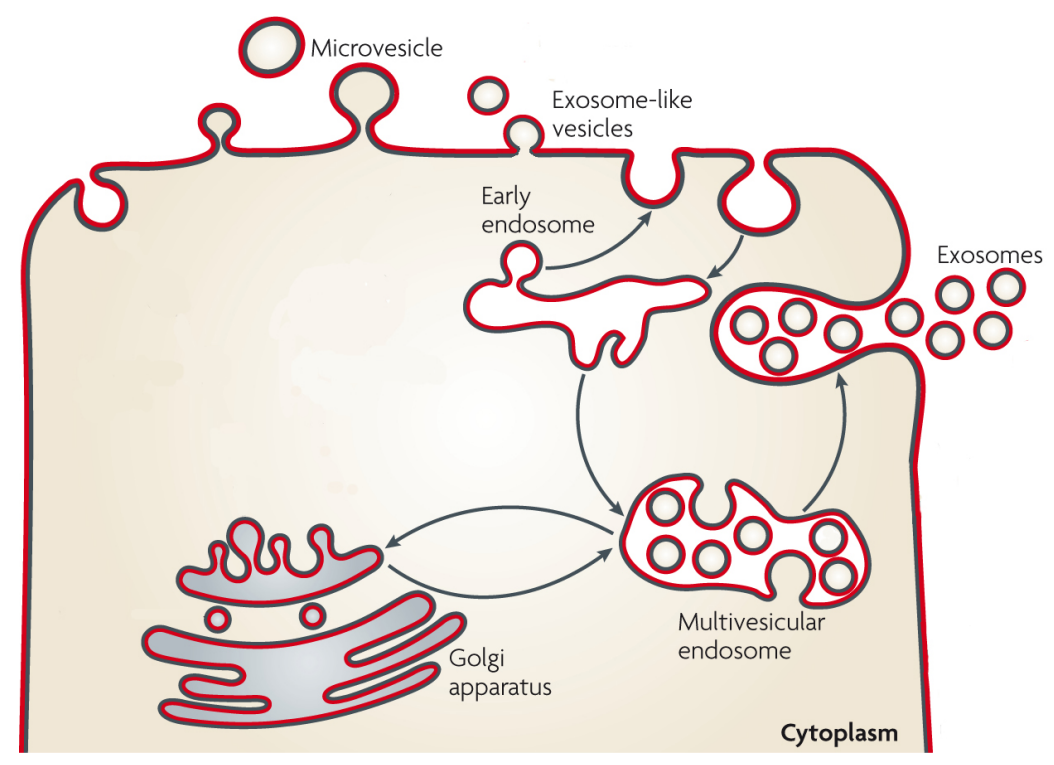

Figure 1.1: Generation and release of exosomes. Cells release different membranous vesicles into their environment. Among those are microvesicles with a heterogeneous size of $100 \mathrm{~nm}$ $1 \mu \mathrm{m}$, which bud from the plasma membrane. Exosomes with a size of $50-100 \mathrm{~nm}$ correspond to intraluminal vesicles of multivesicular endosomes, which are generated by membrane invagination of endosomes. They are released upon fusion of multivesicular endosomes with the plasma membrane. 50 - $100 \mathrm{~nm}$ large exosome-like vesicles can also immediately bud from the plasma membrane. The figure is adapted from Théry et al., 2009 (3). Reprinted by permission from Macmillan Publishers Ltd: Nature Reviews Immunology, copyright 2009. 
to mediate immunological responses in acceptor cells (8). They selectively carry subsets of proteins, lipids, and RNAs $(9 ; 10 ; 11)$ and thus can be involved in signal transduction processes $(12 ; 13 ; 14)$. Moreover, they were recently associated with disease progression $(15 ; 16 ; 17)$.

Exosome secretion is known for numerous cell types in culture, including hematopoetic cells, e.g. B cells, T cells, and mast cells $(8 ; 18 ; 19)$, but also cells of non-hematopoetic origin, such as neurons, oligodendrocytes, and epithelial cells $(20 ; 21 ; 22)$. Comprehensive investigations of exosome function and destination further led to their discovery in mammalian body fluids such as blood plasma, urine, cerebrospinal fluid, and malignant effusions $(23 ; 24 ; 25 ; 26)$ indicating their biological relevance in vivo.

\subsubsection{Morphology and composition}

\section{Morphology}

Exosomes have a characteristic size, density, morphology, and cargo composition. Using these hallmarks, they can be discriminated from other vesicular structures in several assays. Exosomes are purified from conditioned medium and biological fluids by ultracentrifugation at $100,000 \mathrm{~g}(27)$. With this protocol however, other small vesicles with a similar size might simultaneously be collected. To validate the purity of the preparation subsequent methods may be used in addition. Flotation of the vesicle preparation in sucrose gradient ultracentrifugation allows the separation of exosomes and vesicles derived from the endoplasmatic reticulum (ER) and the Golgi apparatus as well as the separation from protein aggregates and other contaminations. Within the gradient, exosomes float at densities of $1.13-1.19 \mathrm{~g} / \mathrm{ml}(8 ; 28)$. In contrast, vesicles from the Golgi apparatus float at $1.05-1.12 \mathrm{~g} / \mathrm{ml}$ whereas ER vesicles are found at $1.18-1.25 \mathrm{~g} / \mathrm{ml}(27)$. Vesicles released during apoptosis float at higher densities between $1.3-2 \mathrm{~g} / \mathrm{ml}$ (29). Exosome-like vesicles with a size of 50-100 $\mathrm{nm}$ can also immediately bud from the plasma membrane instead of being released by MVB-fusion with the plasma membrane in a delayed pathway (30). The discrimination between immediately released exosome-like vesicles and delayed released exosomes is however difficult since both vesicle types are found at densities of $1.13 \mathrm{~g} / \mathrm{ml}$ (31). Exosome morphology can further be studied by electron microscopy, where they appear in a typical 'cup shaped' structure as flattened spheres with a lipid bilayer boundary and a size of $50-100 \mathrm{~nm}$ in diameter $(20 ; 32)$. This is consistent with the morphology and size of ILVs within MVBs (8). 


\section{Protein composition}

The protein content of exosomes has been analyzed with mass spectrometry, Western blotting, immuno-electron microscopy, and flow cytometry $(9 ; 33 ; 20 ; 34)$. In general, the protein profile of exosomes is determined by their parent cells, their origin from the endosomal system and their specific physiological functions. It is not yet completely understood, which roles the resident proteins fulfill. However, many of them seem to be involved in the initial formation of exosomes.

Some subsets of proteins are conserved in all exosomes. This includes members of the ESCRT (endosomal sorting complex required for transport) machinery, e.g. alix and tsg-101, which are essential for MVB generation. Other conserved proteins are members of the cytoskeleton, such as actin and tubulin, and proteins that facilitate vesicle generation, fusion and mobility, e.g. clathrin, annexins, Rab1B, Rab7, and Rab35. Proteins ubiquitously appearing in exosomes also include metabolic enzymes, e.g. peroxidases and enolase-1, the chaperons Hsc70, Hsp70 and Hsp90, and proteins mediating signal transduction, e.g. heterotrimeric $\mathrm{G}$ proteins and several kinases. In addition, adhesion molecules such as integrins and the teraspanins CD9, CD63, CD81, and CD82 are remarkably enriched in exosomes of all cell types $(35 ; 9 ; 27 ; 36)$. Recent studies further used flotillins - proteins associated with lipid-enriched membrane microdomains - as markers for exosomes derived from several cell types $(37 ; 32 ; 38)$.

In contrast, other proteins are selective for exosomes released from a distinct subpopulation of cells. These are most probably involved in cell type specific functions (see Section 1.1.4) and directed into exosomes in a regulated fashion. The transferrin receptor was reported to be released from maturing reticulocytes via exosomes (6). MHC class I and II molecules are most abundant in exosomes derived from cells of the immune system (33). Exosomes from neurons are enriched in glutamate receptor $2 / 3(20)$, and dendritic cells as well as some tumor cell lines release exosomes containing high amounts of the milk-fat-globule EGF-factor VIII (MFGE8), which is proposed to direct exosomes to their target cells $(39 ; 35)$. In addition to proteins, also ribonucleic acids can be specifically incorporated into exosomes. Studies on mast cell derived exosomes identified a subset of approximately 1300 mRNAs and another 120 miRNAs (11).

\section{Lipid composition}

With regard to their lipid composition, exosomes generally seem to resemble the plasma membrane of their donor cells (6). Their membranes are comprised of cholesterol, lysophos- 
phatidylcholine, phosphatidylcholine, phosphatidylethanolamine, sphingomyelin, diglyceride, and phosphatidylserine (PS) (40) and harbor lipid-rich microdomains (37). PS was proposed to promote 'reverse budding' events, in which the outer membrane leaflet corresponds to the extracellular membrane site (35). In line with this, exosomes derived from platelets were found to expose PS at their outer leaflets (41). Lysobisphosphatidic acid (LBPA), a negatively charged lipid abundant in late endosomal compartments, seems to be essential for the formation of ILVs and was found in exosomes released by B lymphocytes $(42 ; 43)$. Depending on their origin, exosomes may be enriched in certain lipids compared to the plasma membrane of the respective parent cells. In exosomes derived from B lymphocytes, the ratio of cholesterol to phospholipids was found to be increased (10). However, this was not seen for exosomes of mast cells and dendritic cells, which instead displayed higher abundance of sphingomyelin and phosphatidylethanolamine (40).

\subsubsection{Biogenesis of exosomes}

\section{Exosomes correspond to intraluminal vesicles of MBVs}

Exosomes are assumed to be generated by inward budding of vesicles from the limiting membrane of late endosomes, giving rise to ILVs within MVBs. Consistent with this model, their membrane orientation reflects that of the plasma membrane and antibodies directed against the extracellular domains of membrane proteins, e.g MHC II, CD9 or CD63, can specifically bind to exosomes $(8 ; 39 ; 34)$. In contrast, proteins within the membrane-enclosed cytosolic core are not detectable with antibodies in exosome whole mounts without prior permeabilization (39). Further verifying their endosomal origin, exosomes contain LAMP-1, tsg-101, and Rab7, but lack proteins of the nucleus, mitochondria, ER and Golgi apparatus. Pulse chase BSAG-labeling and subsequent imaging in electron microscopy allowed to directly track MVBs in the cytoplasm and at their plasma membrane-fusion sites, illustrating the events that result in exosome release $(8 ; 2)$. Nevertheless, the exact mechanisms required for regulation of exosome generation and selective integration of proteins into these structures are yet poorly understood.

\section{ESCRT-dependent protein sorting}

Proteins destined for sorting into specific intracellular structures, such as the plasma membrane or nucleus, exhibit a characteristic signal included in their amino acid sequence (44). For protein sequestration into exosomes however, no unifying sorting sequence has been reported so far. A number of sorting mechanisms are instead suggested to be involved in 
this process. One key pathway seems to use the ESCRT machinery $(45 ; 46)$. In this process, ubiquitin-tagged proteins are recognized by Hrs, which forms a complex with STAM, clathrin and Esp15. Subsequently the ESCRT I component tsg-101 is targeted to the cargo, followed by ESCRT II and ultimately ESCRT III, which initiates membrane budding to form ILVs (46). The protein complexes eventually dissociate, and the members of ESCRT are recycled in a process that involves activity of the AAA-ATPase Vps4 $(47 ; 48)$. It seems that mono-ubiquitination and probably oligo-ubiquitination serves as a signal for targeting proteins into ILVs of MVBs (49), while tagging proteins with multiple ubiqiutin moieties initiates their degradation by the proteasome (50). Exosomal enrichment in the ESCRT components alix, tsg-101, and clathrin $(35 ; 9)$ as well as in ubiquitin ligase c-CBL (18) indicates that this pathway is indeed used for biogenesis of exosomes. One example for cargo, which bind to ubiquitin to be sorted into ILVs, is Vsp23 - a homolog of tsg-101 in yeast (51). However, the entry point into ESCRT sorting might differ for various proteins. The transferrin receptor of reticulocytes does not require ubiquitination but an association with alix to initiate its vesicle incorporation (52). ILV-sequestration of non-ubiquitinated G-protein coupled receptors seems to involve activity of Vps4 and Hrs, but not tsg-101 (53).

\section{ESCRT-independent mechanisms}

In addition to ESCRT, other sorting mechanism are also likely to exist since the transmembrane premelanosomal protein Pmel17, the proteolipid protein (PLP), and CD63 are targeted into ILVs independently of the ESCRT machinery $(54 ; 55 ; 56 ; 57)$. Incorporation of cytosolic proteins into exosomes may be realized by random inclusion of cytosol during endosomal inward budding. In that way ubiquitously expressed proteins such as tubulin, actin, and actin-binding proteins would find their way into exosomes (35). By another mechanism, interaction partners of transmembrane proteins would be co-sorted into ILV. This process was proposed for the chaperons Hsp70 and Hsc70, which were shown to interact with transferrin receptors (52).

\section{Protein oligomerization}

A recent model proposes protein oligomerization and membrane association to be sufficient for cargo transport into exosomes (56). Protein oligomerization may lead to stabilization of membrane microdomains, candidate sites for membrane invagination $(58 ; 2)$. This model is supported by the finding that antibody-induced aggregation of the transferrin receptor of reticulocytes facilitates its translocation from recycling endosomes to MVBs 
(59). Crosslinking and higher-order oligomerization of proteins similarly increase exosomal release of MHC II in lymphocytes (60) and CD43, CD45, and CD59 in Jurkat T cells (56). The latter study, however, focused on exosomal structures that immediately bud from endosome-like domains of the plasma membrane instead of being derived from MVBs. This is a pathway, by which HIV virus particles are released from cells (56).

\section{Lipid microdomain sorting}

A major exosome-sequestration mechanism for other cargoes may be their localization in 'lipid rafts' (37). This would include proteins directly associated with lipid-rich membrane microdomains, such as stomatin, flotillins, MHC II and lyn (37). Several studies indeed confirmed an enrichment of these proteins in exosomes secreted from various cell types $(35 ; 37 ; 32)$. The underlying mechanism of ILV-formation at lipid-rich microdomains may be the emergence of high tensions at the boundary between liquid-ordered and disordered domains that would promote membrane constriction to form ILVs (2).

\section{Ceramide-dependent sorting}

Ceramide was demonstrated to promote lateral phase separation and formation of subdomains in model membranes (61). The cone-shaped structure of this sphingolipid is likely to induce negative membrane curvature, which would result in inward budding of the membrane (2). Exsosomes derived from Oli-neu cells display a marked enrichment in ceramide (57). In these cells, targeting of exosomal proteins into ILVs of MVBs was not dependent on the ESCRT machinery but on ceramide. When ceramide was reduced by inhibition of neutral sphingomyelinase activity, exosome release became decreased (57).

\section{Teraspanin-dependent sorting}

Tetraspanins, which display a high affinity to sphingolipids and cholesterol, also promote the formation of membrane microdomains. By interacting with each other and with various cytosolic and transmembrane proteins, tetraspanins form networks. These could retain MHC II, intergrins, and signaling molecules at plasma membrane-domains and thus include them into exosomes $(62 ; 63)$. In support of this, tetraspanins were shown to be largely present in exosomes of numerous cell types $(35 ; 64)$. 


\subsubsection{Release of exosomes}

Secretion of exosomes into the cellular environment is eventually realized by fusion of MVBs with the plasma membrane. To date very little is known about the regulation of this process and the factors comprising the involved molecular machinery. Speculations on the machinery's composition arose from studies on intracellular vesicle fusion events. Basic components acting in vesicle fusion are soluble factors, such as NSF and SNAP, and membrane proteins, especially SNAREs (65). To secure the specificity of fusion events, the docking membranes have to incorporate distinct vesicle-SNAREs (v-SNAREs) and target-SNAREs (t-SNAREs), respectively. The v-SNAREs VAMP7 and VAMP8 as well as the the t-SNAREs SNAP23, syntaxin-3 and syntaxin-4 were found to regulate exocytosis events during degranulation in mast cells $(66 ; 67 ; 68)$. In respect of lysosome exocytosis, synaptotagmin-7, syntaxin-7, synaptotagmin-2, and Ca2+ levels were determined as crucial regulation factors $(69 ; 70)$. Whether these same components are similarly involved in the mediation of MBV-fusion with the plasma membrane to release exosomes remains unclear and demands further investigation.

\section{Involvement of Rabs}

Another protein family essential for vesicle trafficking, budding and fusion processes at various membrane sites is the Rab family of small GTPases (71). By an shRNA-based screen in HeLa cells, Rab2b, Rab9b, Rab5a, Rab27a, and Rab27b, were identified as possible effectors of exosome generation and release (72). Of those, Rab27a and Rab27b are proposed to participate in docking of MVBs to the plasma membrane. Silencing of Rab27a resulted in an remarkable increase in MVB size and Rab27b knock-down redistributed MVBs towards the perinuclear region. In addition, silencing two effectors of Rab27 induced the same molecular phenotype as seen with Rab27a and Rab27b knock-down (72). Rab35 is another GTPase that was found to be involved in plasma membrane-docking or -tethering of MVBs (36). Rab35 traffics between the plasma membrane and late endosomes/lysosomes in a GDP/GTP-dependent manner. Inhibition of Rab35 accumulates endosomal vesicles intracellularly, and expression of a dominant-negative Rab35 mutant decreases the release of PLP-containing exosomes (36).

\section{Calcium and potassium}

The activity of Rabs might regulate exosome release in concert with intracellular calcium levels, as Rab11 was demonstrated to enhance secretion of exosomes in dependence of 
$\mathrm{Ca}^{2+}$ in a human erythroleukemia cell line $(73 ; 74)$. A Ca ${ }^{2+}$-dependence of exosome secretion has been additionally shown for mast cells (19). Similarly, release of so called 'secretory lysosomes', late endocytic vesicles that fuse with the plasma membrane, is mediated in a $\mathrm{Ca}^{2+}$-dependent manner (75). Neurons can also secrete exosomes in response to $\mathrm{K}^{+}$-induced depolarization (20). These findings indicate that levels of $\mathrm{Ca}^{2+}$ and $\mathrm{K}^{+}$ contribute to the regulation of MVB-fusion with the plasma membrane, although the exact mechanism underlying this process is still elusive.

\section{Other factors}

Other factors proposed to contribute to exosome secretion are citron kinase and the V0subunit of V-ATPase. The RhoA effector citron kinase modulates exocytosis of HIV-1 virions and exosome-like vesicles (stained with hsc70, CD82 and LAMP-1) in HeLa and $293 \mathrm{~T}$ cells (76). The V0-subunit of V-ATPase has been suggested as a mediator of MVB fusion events at the plasma membrane, since it acts during membrane fusion independently of the V0-V1 ATPase complex (77). In support of this, impaired apical secretion of exosomes was reported for a Caenorhabditis elegans strain with mutated V0-subunit (78). The release of exosomes may also be negatively regulated by distinct cellular conditions. During autophagy, for example, MBVs are promoted to fuse with autophagic vacuoles instead of secreting their contents as exosomes (79). Taken together, a variety of factors are likely to act on exosome release, which might depend on type and condition of the cell.

\subsubsection{Function of exosomes}

\section{Molecule disposal}

Originally discovered in reticulocytes, the first function attributed to exosomes was the disposal of obsolete plasma membrane, cytoplasm and proteins (6). During the maturation of reticulocytes into erythrocytes cells release exosomes enriched in transferrin receptor (6). It was shown that thereby cellular protein levels are down-regulated without participation of the lysosomal system. The proteins CD55 and CD59 as well as the GPI-anchored protein acetylcholinesterase are also released via exosomes during reticulocyte maturation $(6 ; 80)$. Acetylcholinesterase is reduced down to $50 \%$ in mature erythrocytes $(6 ; 80)$. During differentiation, red blood cells loose their irregular polyglobulated morphology and acquire the typical biconcave disk shape of erythrocytes (81). Membrane surface area and cell volume have to be reduced in course of this process $(82 ; 83)$. Notably, exosomes were found to contain $\mathrm{Na}^{+} / \mathrm{K}^{+}$-ATPase (6). Exosomal disposal of ion transporters, which 
regulate cell water contents, might thus partially account for the cells' volume decrease. Disposal of molecules through exosomes may also be relevant for other cells types, including those lacking an efficient degradation machinery or neighbouring a drainage system. With their release into body fluids, exosomal cargo could be discarded in a way complementary to their catabolic breakdown within the cell. The discovery of exosomes in body fluids such as urine and blood plasma supports this idea $(24 ; 23)$.

\section{Cargo shuttling}

Some exosomal cargo may rather serve complex functions outside their donor cells instead of being degraded by phagocytes. Intensive research on exosomes during the last two centuries has drawn attention to their role as shuttle systems for cell-to-cell communication. In this function, exosomes transport molecules that are taken up by other cells and induce molecule-specific signaling cascades in the recipient cells. One recent example is the role of exosome-like vesicles in Wnt signal transmission between neurons in Drosophila, where they trigger postsynaptic Wnt signal transduction in the Wnt-receiveing cells (13). However, exosomes are not only employed to spread signal proteins and proteins involved in evoking immune responses (35), but they may also be exploited for the propagation of infectious agents $(84 ; 30)$. Carcinoma cells harboring latent Epstein-Barr virus (EBV) release exosomes, which activate ERK and AKT signaling pathways in recipient cells, probably manipulating the tumor's microenvironment (14). In addition to proteins, exosomes derived from mast cells were recently shown to accommodate mRNAs of approximately 1300 genes and another 120 microRNAs (11). The composition of exosomal RNAs did not reflect that of the donor cells suggesting a specific and selective integration of RNA into exosomes (11). The exosome-integrated mRNA was taken up by recipient cells, which subsequently translated them into proteins (11). This strongly indicates that, besides proteins and lipids, also functional RNA may be exchanged between cells via exosomes.

\section{Mediating immune responses}

Another function of exosomes became highlighted by studying those vesicles derived from antigen presenting cells. B lymphocytes transformed with EBV were found to secrete exosomes that activate human $\mathrm{CD}^{4+} \mathrm{T}$ cell clones in vitro in an antigen-specific, $\mathrm{MHC}$ class II-restricted manner (8). In vivo experiments demonstrated T cell-dependent tumor rejection in mice, which was mediated by exosomes released from dendritic cells (DCs) pulsed with tumor derived peptides (28). These exosomes harbored MHC class I and II 
as well as T cell stimulating cofactors. Interestingly, the immune response induced by DC exosomes was similarly high as that evoked by DCs themselves (28). Many more studies on the nature of antigen presenting cell-derived exosomes followed $(54 ; 85 ; 86 ; 87 ; 88 ; 3)$, making the role of exosomes in immune response to one of the best understood.

\section{Spreading of infectious and neurodegenerative diseases}

In addition to their physiological functions, MVBs and exosomes may be 'highjacked' by tumors, viruses and other infectious agents to mediate their spreading. Conversely to the previously described studies, it was shown that in some cases tumor-derived exosomes can as well facilitate antigen-specific tolerance and apoptosis of $\mathrm{T}$ cells $(89 ; 15)$. Exosomes secreted by murine mammary carcinoma cells promote tumor growth in vivo by suppressing the cytosolic activity of natural killer cells (90). Also retroviruses, such as HIV-1, were proposed to exploit the MVB generation machinery for their propagation. Similarly to other antigens (7), HIV particles can be internalized into MVBs of DCs (91) and are further transmitted to T cells via exocytosis (92). Exosomes may also be involved in the progression of neurodegenerative diseases such as Parkinsons's disease, Creutzfeldt-Jakob disease, and Alzheimer's disease (84; 93; 94). Prion protein (PrP)-expressing cells readily release exosomes harboring both the naturally occurring $(\mathrm{PrPc})$ and the abnormally folded, infectious scrapie (PrPsc) variant of this protein (84). Non-infected cells were reported to newly form PrPsc by conversion of endogenous PrPc after incubation with exosome preparations containing PrPsc in vitro and in vivo (84; 93).

One hallmark of Alzheimer's disease is the processing of amyloid precursor protein (APP) into aggregating $\mathrm{A} \beta$ peptides (95). Minor fractions of $\mathrm{A} \beta$ were found to be released from HeLa and N2a cells via exosomes (17). Accordingly, two exosomal marker proteins, alix and flotillin-1, are detectable within plaques of transgenic mice and Alzheimer's disease patients' brain sections, respectively $(96 ; 17)$.

\subsection{The flotillin proteins}

Independent of their association with exosomes (57), flotillins were first discovered in the year 1997 in two distinct contexts. Claudia Stuermer's group found two proteins upregulated in retinal ganglion cells during the regeneration of axons in goldfish optic nerve lesions. Hence, they were termed reggie-1 and reggie-2 (97). At the same time, Michael 
Lisanti's group isolated the two proteins from mouse lung tissue, where they were associated with triton-insoluble, buoyant membrane fractions. Highlighting their 'floating' behavior, Lisanti's group named them flotillin-2 and flotillin-1 (98). Further investigations revealed flotillins to be evolutionary highly conserved proteins, which are already present in some bacteria, fungi and plant species $(99 ; 100 ; 101)$. The sequence homology of flotillins was determined to be $64 \%$ between fly and human (102) and approximately $90 \%$ between human and other vertrebrates (100).

\section{Tissue expression and cellular localization}

Flotillins are widely expressed among various species, tissues, and cell types. Despite their ubiquitous occurrence, the proteins are especially enriched in certain cell types, such as erythrocytes, neurons, myocytes, and adipocytes (103). Moreover, upregulation of flotillin expression seems to be essential during cell differentiation, formation of cell-to-cell contacts, and in processes of early development and regeneration $(104 ; 105 ; 102 ; 97)$.

Since flotillins are attached to cell membranes, they are predominantly localized to the plasma membrane (106) and are also found in recycling endosomes (107), lysosomes (108), phagosomes (109), and MVBs (37). More recently flotillin-1, but not flotillin-2 was reported to be translocated into the nucleus in a cell cylce dependent manner (110), although its function there is still elusive. At the membrane, flotillins are organized in stable clusters with a diameter of approximately $100 \mathrm{~nm}(106 ; 111)$. They are associated with detergentresistant, liquid-ordered membrane microdomains enriched in cholesterol and sphingolipids (112). These lipid microdomains can be purified biochemically by their flotation in low buoyant sucrose density gradients (113).

\subsubsection{Protein structure}

\section{Membrane association}

Flotillin-1 and flotillin-2 are proteins with a molecular weight of approximately $47 \mathrm{kDa}$ (99). Structural analysis of murine flotillins revealed a $47 \%$ homology and $68 \%$ simliarity between the two isoforms (114). Although flotillins are not spanning the membrane, they are associated via hydrophobic amino acid (aa) regions, which partially incorporate the proteins into the membrane. Their C- and N-termini are faced to the cytosol in a similar pattern as described for caveolins $(115 ; 116)$. Membrane association is mediated through acylation, i.e. myristolation and palmitolation, at distinct $\mathrm{N}$-terminal sites forming anchors into the cytoplasmic leaflet of the membrane (117). Mutations in these regions 
were observed to impair flotillin's membrane association and increase its solubility (117). A more detailed structure of flotillin-2 attached to the membrane is depicted in Figure 1.2.

\section{The SPFH domain}

Flotillins are classified as members of the stomatin/prohibitin/flotillin/hflK/C (SPFH) protein superfamily - proteins harboring an N-terminal SPFH domain of yet unknown function (113). These proteins might have evolved by convergent evolution (100) and are found in numerous pro- and eucaryotic species (114).

\section{The flotillin domain}

The flotillin proteins also contain a unique flotillin domain in their C-terminus that consists of so called EA repeats, protein regions rich in glutamic acid and alanine that are proposed to form three coiled-coil structures $(118 ; 98)$ important for oligomerization of flotillins. It could be demonstrated that flotillin-1 and flotillin-2 form homo- and heterotetramers stabilized by the coiled-coils 1 and 2 (119). This oligomerization is essential for protein stability, since siRNA mediated knock-down of flotillin-2 induces degradation of flotillin-1 via the proteasome (119).

\section{CRAC domains}

In addition to these sequences, flotillin-2 was recently predicted to contain two putative cholesterol recognition/interaction amino acid consensus (CRAC) domains with the primary sequence of (L/V-(X)(1-5)-Y-(X)(1-5)-R/K) spanning aa 120-127 (VEQIYQDR) and aa 157-169 (VYDKVDYLSSLGK) of the protein (120). CRAC domains were first discovered in the peripheral type benzodiazepine receptor (PBR) (121), where their functionality in cholesterol binding was described and a possible role in cholesterol transport into mitochondria was explored (see Section 1.3.2) (122; 123). Mutation of a tyrosine residue in PBR's CRAC domain impaired the translocation and uptake of cholesterol into mitochondria (123). The CRAC domain is also present in caveolin. Peptides containing caveolin's CRAC domain promoted sorting of cholesterol into cholesterol-rich membrane domains to a higher extend than caveolin peptides that did not contain the CRAC domain (124). Similar to their functions in PBR and caveolin, CRAC domains might enable flotillin-2 to bind to cholesterol and recruit it to the plasma membrane. 


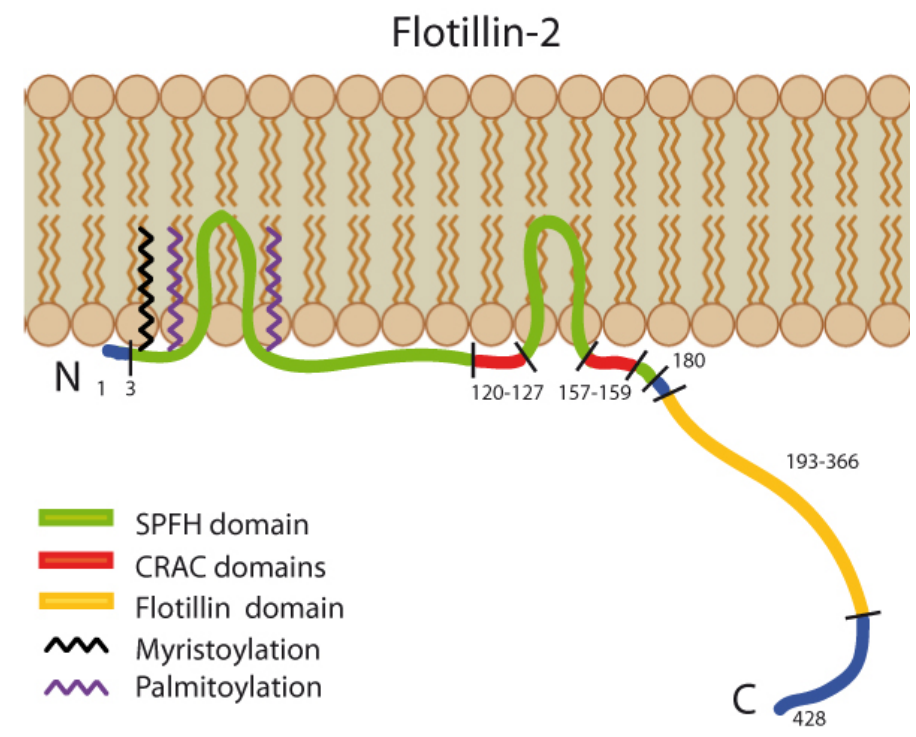

Figure 1.2: Structure of flotillin-2. The figure depicts flotillin-2 attached to the cytosolic site of the plasma membrane facilitated by myristoylation and palmitoylation of its $\mathrm{N}$-terminus. The protein contains several functional domains: the SPFH domain, characterizing flotillin-2 as a member of the stomatin/prohibitin/flotilllin/hflK/C superfamily, two cholesterol recognition/interaction amino acid consensus sequences (CRAC), and a unique flotillin domain important for oligomerization. The figure is adapted from Roitbak et al. (2005), Bauer \& Pelkmans (2006), and Browman et al. (2007) $(120 ; 116 ; 113)$. 


\subsubsection{Protein functions}

\section{Control of cytoskeletal dynamics}

In goldfish, mRNAs of both flotillins were detected in axons of retinal ganglion cells during embryonic development and neuronal regeneration, yet they were lacking in mature axons (97). This phase dependence of protein expression suggests an involvement of flotillins in processes of axonal growth and cytoskeletal remodeling. Participation in cytoskeleton organization is supported by the finding that flotillin- 2 overexpression enhances formation of filopodia in different cell types $(125 ; 117)$. Moreover, tyrosine-phosphorylation of flotillin-2 promotes cell spreading on matrix-coated surfaces whereas siRNA mediated flotillin-2 knock-down reduces cell spreading (126). A direct interaction between flotillin1 and the sorbin homology ( $\mathrm{SoHo}$ ) domain of vinexin family proteins could be detected (127). Vinexins are essential adapters that bind to regulator proteins of cytoskeletal dynamics, e.g. vinculin and afadin, via their SH3 domains (128). Through their association with vinexins, flotillins might serve as platforms facilitating the recruitment of cytoskeletal effector protein complexes to membrane microdomains. Flotillins could therefore regulate processes of cytoskeleton arrangement in an indirect manner (104).

\section{Signal transduction}

Studies investigating the association of flotillins to other proteins by co-localization and co-immunoprecipitation analysis suggest their involvement in various signaling processes (104). However, many of the proposed pathways remain hypothetical since detailed information about the placement of flotillins within signal cascades is still lacking. One of the best studied flotillin-dependent signal transduction mechanism is the insulin stimulated uptake of glucose through GLUT4 glucose transporter proteins. This mechanism was identified in adipocytes as an alternative pathway of glucose uptake, which is independent of PI3 kinase and $\mathrm{PIP}_{3}$ signaling. It involves recruitment and complex formation of c$\mathrm{Cbl} / \mathrm{c}-\mathrm{Cbl}$ associated protein (CAP) at membrane microdomains (129). The recruitment was proposed to be facilitated by the interaction of CAP's SoHo domain with hydrophobic domains of flotillin-1 $(129 ; 130)$. More recent studies, however, suggest a role of flotillin-1 in insulin stimulated glucose uptake independent of an interaction with CAP (131).

Another example of flotillin mediated signal transduction arises from studies of epidermal growth factor (EGF) signaling. Cell stimulation with EGF results in the phosphorylation of flotillin-2 at T163 via Src kinase. The protein is then translocated from the plasma membrane to late endosomes, where it partially co-localized with the EGF receptor (126). 
This suggests that flotillins participate in the signaling of various growth factors (132).

\section{Mediation of endocytosis}

Glebov and colleagues recently proposed a role of flotillins in mediating a novel clathrinand caveolin-independent endocytic pathway (133). The authors found flotillin-1 to be localized to punctuate regions of the plasma membrane that budded into the cell but did not resemble clathrin-coated pits or caveolae. Flotillin-1 was further found in endocytic intermediate structures, which additionally contained GPI-linked proteins and cholera toxin B. Moreover, when flotillin-1 expression was knocked-down by siRNA, the endocytosis of GPI-linked proteins and the clathrin-independent uptake of Cholera toxin B were both inhibited (133). A flotillin-1/flotillin-2-dependent induction of plasma membrane curvature, invagination and budding to form intracellular vesicles was also reported by Frick and colleagues (134).

\section{Other functions}

The unifying key elements of flotillin functions seem to be recruitment of other proteins to lipid-rich membrane microdomains and facilitation of protein complex formation. These might also be relevant for other processes, as flotillins were further proposed to be involved in the proliferation of cancer cells (110) and the maturation of phagosomes to phagolysosomes (109). Moreover, upregulation of flotillin-1 expression is associated with the occurrence of type 2 diabetes and Alzheimer's disease (135; 94).

\subsection{Cholesterol in health and disease}

The natural steroid cholesterol consists of four transfused rings, two methyl groups, a hydroxyl group and an isooctyl side chain (see Figure 1.3) (136). As one of the major lipids produced by animals, it is indispensable for the structural and functional integrity of each cell. Due to its polarity, cholesterol integrates into cell membranes and regulates their fluidity and permeability (137). The lipid is also a key component of myelin. This explains its enrichment in brain, which is demonstrated by the fact that the brain comprises only $2 \%$ of total body weight, whereas it contains $25 \%$ of total body cholesterol (138; 139). Moreover, cholesterol serves as a precursor for the generation of bile acids, steroid hormones, and oxysterols (140). 


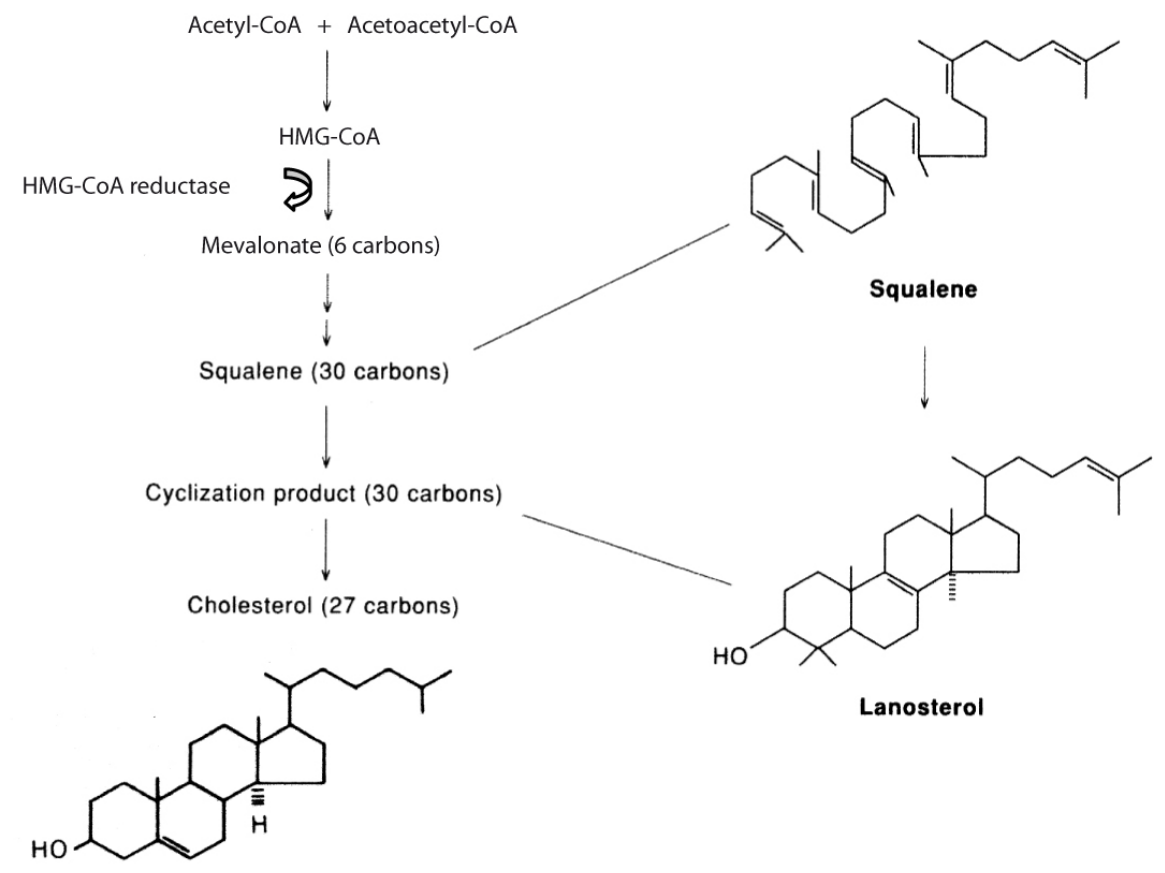

Figure 1.3: Basic scheme of cholesterol synthesis. Please find the detailed description of cholesterol synthesis in the main text. The figure is adapted from Vance and Bosch, 2000 (141). Reprinted by permission from Elsevier, copyright 2000. 


\subsubsection{Mechanisms of cholesterol synthesis and uptake}

\section{Cholesterol synthesis}

All cholesterol that is incorporated into the cell is either newly synthesized or taken up as low-density lipoproteins (LDL). Cholesterol is synthesized de novo in cells of the brain, the liver, and other extrahepatic tissues. This process is especially important in cells of the nervous system (142), as LDLs are not able to cross the blood-brain-barrier. They can therefore not supply the brain with cholesterol circulating in the blood flow. Synthesis of cholesterol is a stepwise reaction involving activity of various enzymes (see Figure 1.3) (143). Its anabolism starts with the condensation of acetyl-CoA and acetoacetyl-CoA to 3-hydroxy-3-methylglutaryl-CoA (HMG-CoA). Subsequently, the molecule is reduced to mevalonate by HMG-CoA reductase, a multispan membrane protein in the ER. This is the irreversible, rate limiting process in cholesterol synthesis. It can be impaired by the administration of statins, which act as HMG-CoA reductase inhibitors. Over the intermediate products isopentenyl pyrophosphate, farnesyl pyrophosphate, and squalene, lanosterol is formed, which is finally converted into cholesterol (141).

Cholesterol synthesis is regulated in a homeostatic manner by direct negative feedback. Intracellular cholesterol levels are detected in the ER by an integral membrane protein complex of sterol-regulatory element-binding proteins (SREBPs) and SREBP cleavage activating protein (SCAP) (144). Upon lowering of cholesterol levels, this SREBP/SCAP complex is released from the ER and migrates to the Golgi apparatus (145). Here, SREBPs are cleaved by the two enzymes S1P and S2P, which are in turn activated by SCAP (146). The cleavage products migrate into the nucleus, where they act as transcription factors. They bind to sterol regulatory elements and promote the expression of proteins involved in cholesterol homeostasis, e.g. LDLRs and HMG-CoA reductase (147).

\section{Uptake of LDL}

A second source of cholesterol is the uptake of LDLs from the blood. LDL-cholesterol enters the cell via clathrin-dependent endocytosis upon binding to LDL receptors at the plasma membrane of recipient cells (148). Through clathrin coated pits, LDLs are sorted into a hydrolytic compartment of the endosomal system, where cholesteryl esters of the LDLs are hydrolyzed by acid lipase (149). Subsequently, free, unesterified cholesterol is shuttled into late endosomes (150).

As stated before, the brain and spinal cord are largely excluded from blood-LDL uptake (151). However, it could be shown that the CNS expresses various apolipoproteins, mainly 
apoE, as well as proteins of the LDL receptor family and the ATP-binding cassette family $(152 ; 153)$. ApoE of the the brain is mainly produced by glial cells. It was therefore proposed that cholesterol can be released by glial cells as apoE-containing lipoproteins, which are transported to neurons and internalized via proteins of the LDL receptor family (154).

\subsubsection{Intracellular cholesterol pathways}

\section{Intracellular trafficking}

Once cholesterol is incorporated into the cell, it enters a multitude of pathways (see Figure 1.4). These regulate intracellular distribution and homeostasis of cholesterol or shuttle the lipid as a precursor into metabolic processes. De novo synthesized and LDL-derived cholesterol can both be integrated in the plasma membrane (149). From the plasma membrane cholesterol becomes available for retrograde transport into the ER (155). This pathway can be promoted by loading of cholesterol to the cell (156) and might involve a caveolin-1 dependent, but clathrin independent vesicle transport $(157 ; 158)$.

Another fraction of plasma membrane-cholesterol is recycled back to late endosomes by endocytic processes (159). The two proteins NPC1 and NPC2 shuttle the lipid from late endosomal and lysosomal compartments to the ER and Golgi apparatus (see Section 1.4.1). In the ER, cholesterol is converted into cholesteryl esters (CEs) by the resident enzyme acyl-coenzyme A:cholesterol acyltransferase (ACAT) (160). Cholesterol does not increase transcription of the enzyme but serves as a substrate for allosteric activation of ACAT (161). The emerging CEs are stored in the cytosol as lipid droplets. In a counteracting process, cholesteryl ester hydrolase $(\mathrm{CEH})$ catalyzes their conversion back into free cholesterol (162). Both conversion processes take place simultaneously establishing a balance between free and esterified cholesterol within the cell. Under conditions of cholesterol depletion, ACAT becomes inactivated leading to increased net release of free cholesterol from CEs. This cholesterol is transported to the plasma membrane, from where it can be released via formation of HDLs (163).

The further distribution of cholesterol is mediated through different carrier agents, including NPCs and specific lipid-binding proteins, e.g. proteins containing the START domain or oxysterol binding protein-related proteins. Both classes may be responsible for nonvesicular transport of cholesterol to various destinations $(164 ; 165 ; 166)$. Vesicular transport of cholesterol seems to additionally involve the small GTPases Rab7 and Rab9 as overexpression of these proteins partially ameliorates cholesterol accumulation caused 


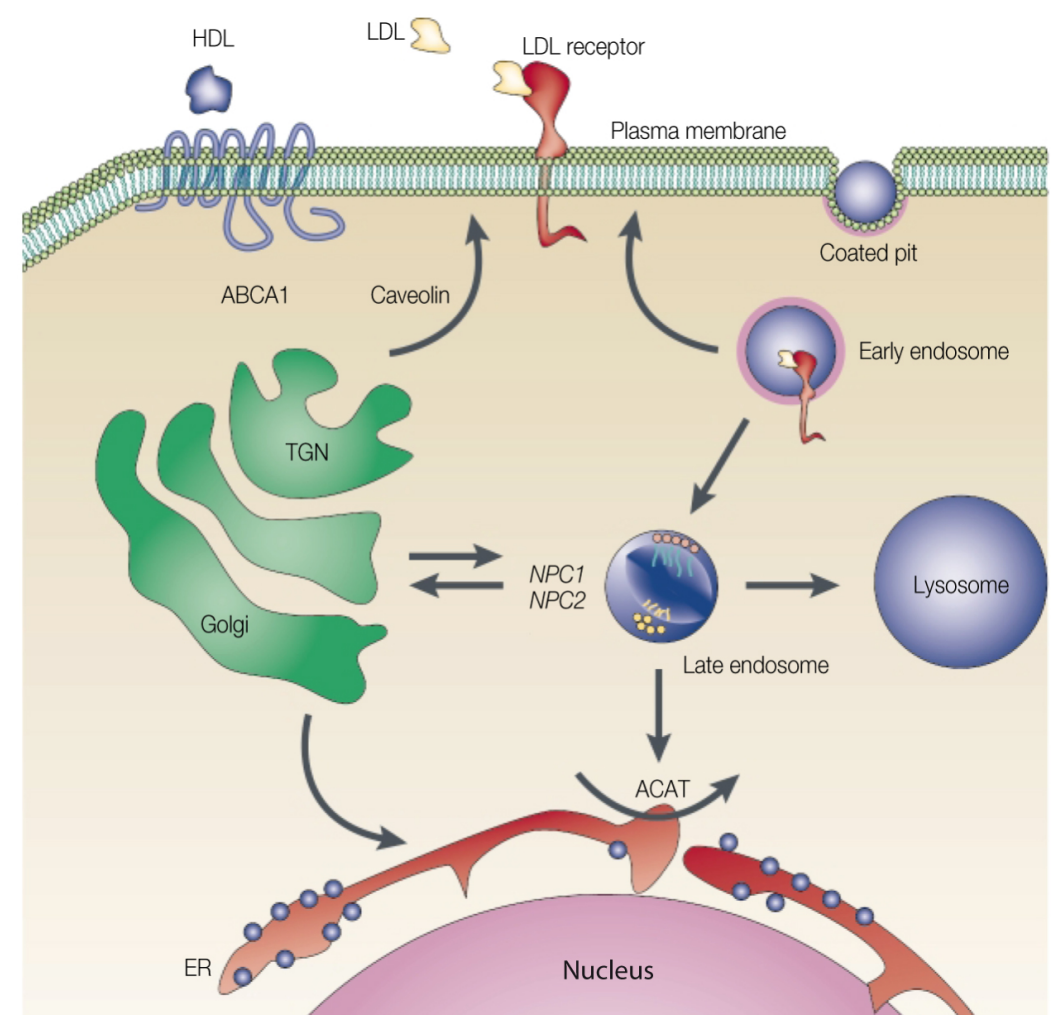

Figure 1.4: Cellular cholesterol pathways. The figure depicts major pathways of cholesterol trafficking within the cell. Cholesterol is synthesized de novo within the endoplasmatic reticulum (ER) or taken up as low-density lipoproteins (LDL) via LDL receptors and formation of coated pits. Along its way to late endosomal compartments, it becomes hydrolyzed by acid lipase. Free cholesterol is shuttled to the plasma membrane by mechanisms involving NPC1 and NPC2 or caveolin. NPC1 and NPC2 also facilitate cholesterol translocation to the ER and Golgi apparatus. Superfluous cholesterol is esterified by acyl-coenzyme A:cholesterol acyltransferase (ACAT) in the ER and stored as lipid droplets in the cytoplasm. For cholesterol efflux, the lipid is secreted from the cell via $A B C A 1$ and formation of high-density lipoproteins (HDL). The figure is adapted from loannou, 2001 (140). Reprinted by permission from Macmillan Publishers Ltd: Nature Reviews Molecular Cell Biology, copyright 2001. 
by loss of functional NPC proteins (167).

\section{Formation of oxysterols and steroid hormones}

Not only the distribution of cholesterol but also its metabolic fate underlies a strong regulation. Cholesterol serves as an important precursor for sterol derivatives, that fulfill tissue specific functions in the organism (140). A fraction of the lipid is oxydized into a variety of hydroxycholesterols. During the synthesis of bile acids, $7 \alpha$-hydroxycholesterol is a crucial intermediate, which is generated by $7 \alpha$-hydroxylase, a rate-limiting enzyme specifically located to the ER of hepatocytes (168). 24(S)-hydroxycholesterol is the most prominent oxysterol in the brain, where it has a special importance for cholesterol efflux (169) (see Section 1.3.3). Other oxysterols, such as 25-hydroxycholesterol and 27-hydroxycholesterol, are involved in the maintenance of cholesterol homeostasis acting on SREBP processing and ABC1 mediated lipid efflux (170; 171).

Cholesterol additionally serves as a precursor for all steroid hormones produced by the organism. The rate-limiting step of steroid hormone generation is the translocation of cholesterol into mitochondria. This is corporately facilitated by two proteins: the steroidogenesis acute regulatory protein (stAR), a member of the START domain proteins, and the CRAC domain containing peripheral benzodiazepine receptor (PBR) (172). Once arrived at the inner mitochondrial membrane, cholesterol is converted into pregnenolone by CYP11 in a step-wise reaction generating three hydroxycholesterol intermediates (173). Subsequently, pregnenolone is shuttled out of the mitochondria into the ER, where it can be catalyzed into any other steroid hormone by the action of several enzymes (158).

\subsubsection{Mechanisms of cholesterol efflux}

\section{General efflux of cholesterol}

To preserve cholesterol homeostasis, superfluous free cholesterol has to be withdrawn from cellular processes. One possibility is its conversion into cholesteryl esters (CEs), which are stored as lipid droplets and can be reactivated if needed (see Section 1.3.2). However, cells are not able to degrade cholesterol. Excess cholesterol is secreted from peripheral tissues into the blood stream. From there, it is taken up by cells of the liver, adrenal glands and other steroid hormone-forming tissues in a process described as 'reverse cholesterol transport' (174). The predominant transporters involved in this process are high-density lipoproteins (HDL), globular particles generally composed of phospholipids and the apolipoproteins A-1 and A-2 that carry cholesterol and CEs (174). Several 
pathways are known, by which cholesterol is shuttled out of the cell. Cholesterol can diffuse passively through the plasma membrane. This process is driven by the force of a concentration gradient of cholesterol between the cell surface and surrounding mature HDLs (175). Secondly, caveolins transport cholesterol from the ER to the plasma membrane, where the lipid becomes enriched and can be taken up by HDLs (176). In a third way, scavenger receptor B1 (SC-B1) enhances the cholesterol flux between cells and HDL (177). Finally, the cell can also generate new HLDs by assembling liver-derived apoA-1 and donor cell-derived phospholipids and cholesterol. The ABC transmembrane protein ABCA1 is a key player in this process (178). Cholesterol loaded HDLs bind to the HDL receptor SP-B1 localized on the plasma membrane of hepatocytes and steroidogenic cells (179). Mediated through this receptor, CEs are taken up into the endosomal system where they can be hydrolysed to free cholesterol. Cholesterol taken up by steroidogenic cells is re-utilized for the synthesis of steroid hormones (179). In contrast, cholesterol internalized into hepatocytes serves as a precursor for the synthesis of bile acids and can be excreted from the organism via the bile duct.

\section{Cholesterol efflux in the CNS}

Cholesterol efflux from the brain makes an exception to the previously described processes. All cholesterol destined for secretion from neurons first has to be converted into 24(S)-hydroxycholesterol by cholesterol 24-hydroxylase (180; 169). By hydroxylation of cholesterol's isooctyl side chain, the molecule is promoted to pass through the lipid bilayer of the plasma membrane much faster than it does in its unmodified form (181). How this mechanism works in detail is still elusive. Almost all cholesterol 24-hydroxylase is synthesized by a small subset of neurons including the pyramidal cells of the cortex and the Purkinje cells of the cerebellum (169). Glial cells are thought to mediate cholesterol efflux by handing the lipid to neurons via apoE-containing lipoproteins (182) (see Section 1.3.1). Once oxysterols reach the blood plasma, they are cleared by the liver.

\subsubsection{Role of cholesterol in disease}

Any impairment of the here described pathways of cholesterol anabolism, trafficking and metabolism can be detrimental to the viability of cells and organisms. Dysfunctions might either be caused by an imbalanced dietary cholesterol supply or by inherited mutations affecting proteins involved in the regulation of cholesterol homeostasis. 
Tangier disease and familial HDL deficiency are rare autosomal recessive diseases caused by mutations in $\mathrm{ABCA} 1$, a member of the $\mathrm{ABC}$ transporters family, which is crucial for cellular cholesterol efflux by generation of HDLs $(183 ; 184)$. Patients do not produce apoA-1 and display a severe reduction in HDL levels (178). As a consequence, cholesteryl esters accumulate in reticulo-endothelial cells, which increase the patients' susceptibility for arteriosclerosis (137).

Mutations in different cholesterol interacting proteins are associated with Alzheimer's disease. For sporadic forms of Alzheimer's disease, the best-established genetic risk factor is the presence of apoE in its $\epsilon 4$ allel (185). Meta-analysis identified mutations causing decreased activity of ACAT to lower the risk for familial forms of Alzheimer's disease (186).

Investigation of such inherited diseases has largely contributed to the understanding of basic mechanisms underlying cholesterol transport and metabolism.

\subsection{Niemann-Pick type $\mathrm{C}$ disease}

\section{History}

In the late 1920's, the pioneering work of Albert Niemann and Ludwig Pick lead to the description of a heterogeneous group of disorders summarized as 'Niemann-Pick diseases'. They all share symptoms of liver and spleen enlargement, as well as an accumulation of sphingomyelin in reticuloendothelial and peripheral tissues. Three centuries later, A. Crocker developed a classification into 4 groups - A to D - based on clinical criteria and the level of sphingomyelin accumulation in cells (187). Niemann-Pick types A an B are characterized by a genetic acid sphingomyelinase deficiency $(188 ; 189)$. Niemann-Pick types C and D (NPC and NPD) display normal levels of sphingomyelinase (190), but have mutations in the genes encoding for the cholesterol transport proteins NPC1 and NPC2. The involvement of NPC1 and NPC2 was discovered in a mouse strain, which accumulated vast amounts of free cholesterol in tissue when kept on high cholesterol diet (191). This strain later served as a model for studying NPC disease (192). NPD is a rare allelic variant of NPC solely found in a population of Nova Scotia $(193 ; 194)$.

\section{Definition}

NPC is defined as an autosomal recessive inherited lipidosis, mainly characterized by progressive demyelination and neurodegeneration (195) due to major abnormalities in traffick- 
a

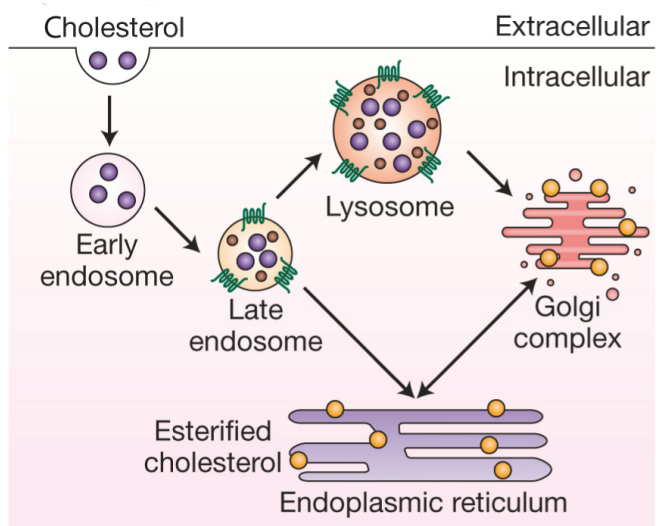

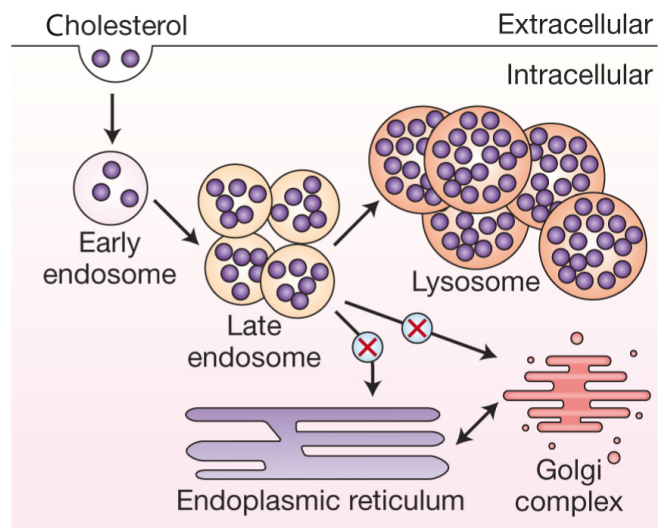

( NPC1/NPC2 deficiency

Figure 1.5: Cholesterol accumulation in NPC. The figure illustrates intracellular cholesterol trafficking mediated by NPC1 and NPC2. (a) Translocation of unesterified cholesterol from late endosomes and lysosomes to the ER and Golgi complex is facilitated by transmembrane NPC1 and soluble NPC2 that are resident in late endosomal/lysosomal compartments. In the ER, cholesterol is esterified by ACAT activity and cholesteryl esters are stored as lipid droplets in the cell. (b) Mutations leading to loss-of-function of either of the proteins cause cholesterol to accumulate within late endosomes and lysosomes whereas it is depleted from other cellular compartments. The figure is adapted from Pacheco and Lieberman, 2008 (203). Copyright Cambridge Journal, reproduced with permission.

ing of cholesterol and sphingolipids, which are eventually accumulated in late endosomes and lysosomes (see Figure 1.5) $(196 ; 197)$. The disease is relatively rare, occurring with a frequency of 1 in 100,000-150,000 births $(198 ; 199)$. Nevertheless, the frequency can be much higher in isolated populations. Families of French Acadians in Nova Scotia and families of upper Rio Grande Valley Spanish have carrier rates of 1 in 10-30 (193; 200). In $95 \%$ of all cases, including those of NPD, mutations in the npc1 gene cause the disease (200; 201; 202), whereas mutations in npc2 account for only $5 \%$ (200).

\section{Clinical manifestation}

NPC is predominantly characterized by late endosomal and lysosomal accumulation of cholesterol $(196 ; 197)$, sphingomyelin, bis(monoacylglycero)phosphate (204), and the gangliosides GM2 and GM3 (205; 206; 207), which occurs predominantly in liver and spleen. In the brain, mostly glucosylceramide and lactosylceramide are found to be co-sequestered 
with cholesterol. Due to this vast accumulation of lipids, neuronal and visceral tissues are largely altered in their morphology and vital functions. This results in a variety of clinical symptoms with disease onset ranging from infantile to late adulthood. Histological hallmarks in the CNS are a loss of neurons, especially in Purkinje cells of the cerebellum, swollen neurites, and progressive demyelination (208). Clinical symptoms include supranuclear ophthalmoplegia cataplexy, other motor defects, seizures, and progressive dementia, as well as psychiatric symptoms such as psychosis (209). In infantile or juvenile disease onset, hepatoslenomagaly and development disturbances are common.

In 2010, miglustat has been approved as a pharmacological, symptomatic therapy (210) by the German authorities. However, diagnosis is difficult due to the variety of symptoms, and it is essentially made late in the course of disease.

\section{Diagnosis}

As a diagnostic assay of NPC, cultivated fibroblasts from skin biopsies of patients are tested with filipin, a polyene antibiotic, which specifically binds to unesterified cholesterol (195). Fibroblasts from NPC patients display a strong perinuclear, vesicular filipin signal in fluorescence microscopy. This typical staining pattern is observed in $80-85 \%$ of all cases, thereby the test is regarded as the most sensitive and specific for NPC, besides genetic analysis.

\subsubsection{Structure and function of the NPC1 and NPC2 proteins}

\section{Structure of NPC1}

To understand the mechanism of NPC1 and NPC2 mediated cholesterol transport, the NPC protein structures were analyzed in detail and scanned for functional domains. NPC1 is a transmembrane glycoprotein consisting of 1278 amino acids (aa). It localizes mainly in Rab7 and Rab9-positive late endosomes, but also associates with lysosomes and the Golgi apparatus (211). Comprised of 13 hydrophobic helices and flanked by a luminal N-terminus and a cytosolic C-terminus, NPC1 is supposed to span the membrane in three large luminal loops and several smaller luminal and cytoplasmic loops (212). Structural analysis of the protein revealed the existence of a sterol-sensing domain (SSD) at aa 616791, a protein segment faced to the cytosol (144). This SSD has a sequence homology with proteins largely involved in the regulation of cholesterol homeostasis, the SCAP, HMGCoA reductase, and 7-dehydrocholesterol reductase (see Section 1.3.1) (212). Loss or gain of function mutations of the SSD effect trafficking of LDL-derived cholesterol (213). 
NPC1 additionally contains an N-terminal domain (NTD) spanning aa 25-264, which includes a leucine-zipper motif (212), as well as another luminal domain spanning aa 8551098. The latter is a cystein-rich region with a ring-finger motif and zinc binding activity (212), which possibly facilitates the interaction with other proteins. This region is highly glycosylated, protecting the protein from proteolytic degradation (214). Interestingly, approximately $30 \%$ of all NPC patients display mutations in this cystein-rich luminal domain (199). In total over 170 different mutations in the NPC1 protein were found in patients of NPC, including insertions, deletions, duplications, and missense mutations (215).

\section{Structure of NPC2}

Although NPC1 and NPC2 both are involved in cholesterol trafficking, they share no sequence identity. The small glycoprotein NPC2 with a molecular weight of approximately $16 \mathrm{kDa}$ consists of only $132 \mathrm{aa}$. It is found as a soluble protein in the lumen of lysosomes, displaying high binding affinity for cholesterol $(216 ; 217)$. Analysis comparing the sequence of NPC2 to that of related vertebrate proteins identified 4 evolutionary constrained regions (ECR) A-D. ECR A seems to be important for secretion of NPC2 and the ECRs B and $\mathrm{C}$ are supposed to promote interaction with other proteins, whereas ECR D is mainly involved in cholesterol binding (218).

\section{Cholesterol transport by NPC1 and NPC2}

Unraveling the mechanism, by which cholesterol is transported from lysosomes to the ER, Golgi apparatus, and plasma membrane, persisted to be a challenging task over the last years. Although various models were proposed $(214 ; 218)$, the exact course of events is still elusive. It is not fully understood how the interaction of NPC1 and NPC2 is regulated under physiological and disease conditions, and whether cholesterol is transported from NPC1 to NPC2, or vice versa. A model of NPC1/NPC2 mediated cholesterol translocation has been proposed recently.

Supported by in vitro studies, this model assumes a direct binding of NPC1 to cholesterol through its NTD $(219 ; 220)$. It was shown that 25-hydroxyl cholesterol competes with cholesterol for this NPC1 binding site, suggesting lipids to mutually regulate their binding to and transportation via NPC1 (219). The authors conducted comparative in vitro studies on the interaction of NPC1 and NPC2 proteins with $\left[{ }^{2} \mathrm{H}\right]$-cholesterol and found NPC2's NTD to rapidly donate and accept liposome derived cholesterol, whereas the NTD of NPC1 acted slowly. They could, however, enhance the bidirectional cholesterol transfer 
between liposomes and NPC1's NTD over 100 times by adding NPC2 (221). NPC2 was only able to promote cholesterol transfer between liposomes and NPC1's NTD when it bound to cholesterol (221). Summarizing these data, a 'handoff' model was suggested, in which NPC1 and NPC2 directly bind to cholesterol and cooperatively regulate its egress from lysosomes. NPC2 probably binds free cholesterol with its hydroxyl group exposed to the lysosomal lumen. It subsequently delivers cholesterol with reversed orientation to NPC1, which is possibly organized as a homodimer $(220 ; 222)$. Cholesterol could then enter the membrane with its hydrophobic side chain (223). In this model, mutation of either of the two proteins would result in the same molecular phenotype, namely an accumulation of cholesterol in lysosomes. In support of this, NPC1 and NPC2 single or double mutants display similar characteristics in terms of disease onset, progression and pathology in genetic studies (224).

\subsubsection{Molecular mechanisms of NPC pathology}

In cells lacking functional NPC1 or NPC2, cholesterol accumulates in late endosomes and lysosomes. How the pathology of NPC is mediated on a molecular basis, is not well understood. Despite great effort in disease research, it is not completely clarified whether lysosomal trapping of cholesterol itself directly causes the molecular toxicity. It is also thinkable that indirect consequences of lipid accumulation or NPC protein deficiency are the prime causes of disease. Furthermore, the question has to be resolved why NPC is predominantly a disease of the central nervous system (CNS) and why neurons are especially vulnerable, although expression of NPC proteins is ubiquitous and not restricted to the brain. Glycosphingolipids display cellular accumulation in NPC as well. It is therefore not ruled out that lipids other than cholesterol might be the major offending metabolites in NPC. In support of this, treatment with cholesterol lowering drugs does not protect from neuropathology albeit free cholesterol levels in the liver are reduced (225).

\section{Accumulation of gangliosides}

Net levels of gangliosides but not of cholesterol are increased in NPC1-deficient brains (226). Due to this finding, a model was proposed, in which cholesterol accumulation occurs only as a secondary effect of glycosphingolipid accumulation (227). To support this hypothesis, inhibitors of glucoceramide synthase, e.g. N-butyldeoxynojirimycin (NBDNJ), were tested in NPC. These inhibitors were previously proven to deplete neuronal glycosphingolipid in an animal model of glycosphingolipid storage disease (228). Applica- 
tion of NB-DNJ to NPC1-mutant mice and cats delayed the onset of neurological symptoms, increased the life span, and reduced ganglioside accumulation of the individuals (229). When NPC1 null mice were crossed with mice deficient in ganglioside biosynthesis, the double knock-out individuals did not accumulate gangliosides in the brain. Yet there was no improvement of life span or neurological phenotype compared to NPC1 null mice (230). It is therefore still questioned whether and how levels of gangliosides influence the progression of NPC.

\section{Cholesterol accumulation in NPC brains}

Although cholesterol accumulation is a prominent feature of cells lacking functional NPC proteins, studies investigating brain cholesterol levels led to contradicting results. On one hand, cholesterol levels are significantly raised in cultured glial cells and neurons from NPC1 null mice (231). When measuring new born mice brains, in which myelination is still incomplete, cholesterol contents are higher in NPC1-deficient than in wild-type mice (232). On the other hand, total cholesterol contents are not increased in brains of NPC1deficient adult mice and humans. They even decline with ongoing aging of the individuals $(233 ; 232)$. The observation that overall amounts of brain cholesterol are reduced instead of increased in the course of NPC disease can be explained by a progressive demyelination (234). Since cholesterol is a major component of myelin, the lipid is released from the brain together with myelin. This could mask the accumulation of cholesterol in neurons.

\section{Reduction of anterograde cholesterol transport in neurons}

Deficiency in proper targeting and utilization of cholesterol within the cell can result in profound cellular dysfunction. Investigation of lipid distribution within neurons revealed an accumulation of cholesterol in late endosomes, whereas it was depleted at the plasma membrane $(196 ; 235)$. This result is of great interest since cholesterol largely participates in the regulation of membrane fluidity and permeability. NPC1-deficient neurons further have a reduced cholesterol content in distal axons (231), which results from an impaired anterograde transport shuttling cholesterol from cell bodies to distal destinations (236). Therefore, the NPC proteins seem to have an additional axon-specific function (237). This is supported by the finding that NPC1 is present in distal axons and recycling endosomes of the pre-synaptic nerve terminal, while NPC2 is present in distal axons and post-synaptic densities $(238 ; 236 ; 239 ; 182 ; 240)$. 
Similar cholesterol contents are present in synaptic vesicles isolated from brains of NPC1deficient and wild-type mice. However, the relative amounts of synaptophysin and synaptobrevin are changed in NPC1 null neurons. These two proteins form a complex, which is critical for synaptic vesicle recycling and depends on plasma membrane cholesterol levels $(241 ; 237)$. A scenario was proposed, in which cholesterol depletion at the plasma membrane of distal axons leads to decreased formation of the synaptophysin/synaptobrevin complex in NPC (240). Loss of functional NPC proteins would decrease plasma membrane cholesterol and thus be detrimental to proper synaptic vesicle recycling and release of neurotransmitters. In support of this model, NPC null neurons display dramatic morphological changes including axonal swellings, meganeurites, and ectopic dendrites (242; 243). These changes result in progressive 'dying back' of axonal nerve terminals and dendrites prior to neuronal cell death $(244 ; 245)$.

\section{Cholesterol deprivation of mitochondria}

Since cholesterol also serves as a substrate for bile acid and steroid hormone production, its late endosomal/lysosomal trapping deprives the cell from essential metabolites. Cholesterol entry into the mitochondrial matrix, where it is converted into pregnenolone, is the rate limiting process in steroid hormone biosynthesis. In a murine model of NPC1, synthesis of steroid hormones was found to be reduced, which is possibly due to reduced levels of cholesterol reaching the mitochondria. More recently, comparison of neurosteroid levels in wild-type and NPC1-deficient mice confirmed this finding, showing lowered neurosteriod levels in NPC null mice. In an attempt to restore steroid hormone levels, NPC-mutant mice were treated with allopregnanolone. This trial resulted in a prolonged life span, delayed onset of neurological symptoms and an improvement of neurological functions (246).

\section{Impairment of endosomal trafficking}

Another phenomenon seen in NPC is a defective trafficking of metabolites and effectors along the endosomal system. Reduced mobility of endosomal tubulovesicular compartments was observed in fibroblasts lacking NPC1 (247). Moreover, NPC1 deficiency causes impaired recycling of material from early endosomes to the plasma membrane $(204 ; 248)$ and mistargeting of proteins, such as the TrkB receptor, leading to reduction in neurite outgrowth and branching (249). Small GTPases (Rabs) are key players in intracellular vesicle trafficking. Overexpressing Rab7 or Rab9 in NPC null fibroblasts promotes cholesterol transport from endosomes to the ER and Golgi apparatus and restores $60-70 \%$ of 
the NPC phenotype (167). These vesicular dysfunctions demonstrate that a lack of functional NPC1 or NPC2 not only leads to lipid accumulation in late endosomes, but is also detrimental to vesicular organization processes.

\subsection{Aims of the study}

This study was conducted to analyze whether cells use the exosome pathway for secretion of cholesterol into the extracellular space. Thereby exosomes could contribute to the maintenance of the cellular cholesterol homeostasis.

We first wanted to identify the potential of cells to adjust their exosome release in respect to cholesterol levels. We therefore studied exosome formation and release in cells cultured under conditions of cholesterol depletion or cholesterol loading.

Secondly, we were interested in the molecular mechanism by which exosomal cholesterol release is mediated. We therefore focused on the interaction of cholesterol and flotillin-2, an exosomal marker protein. Flotillin-2 contains two cholesterol recognition/interaction amino acid consensus (CRAC) sequences (120), which might mediate cholesterol binding. This process could direct the protein to cholesterol-enriched membrane microdomains, from where flotillin-2 dependent endocytosis might direct cholesterol into intraluminal vesicles of MVBs, which are released as exosomes. To validate the functionality of flotillin's CRAC domains, flotillin-2 constructs bearing mutations in one or both CRAC domains were examined for their redistribution to intracellular vesicular structures and their release via exosomes. Since flotillin-2 has been shown to participate in the regulation of cholesterol distribution (120), the importance of flotillin-2 in facilitating exosomal cholesterol release was tested by selective down-regulation of the protein.

In a third part, we studied the physiological relevance of exosomal cholesterol secretion in the lysosomal storage disorder Niemann-Pick type C (NPC). This disease is caused by mutations in either NPC1 or NPC2 protein, which translocate cholesterol from late endosomes/lysosomes to the ER, Golgi apparatus and plasma membrane. As a consequence, unesterified cholesterol accumulates within late endosomes/lysosoms $(196 ; 197)$. We investigated whether exosomes could partially bypass this cholesterol traffic block by shuttling accumulated cholesterol out of the cell. Therefore, cells with drug induced molecular NPC pathology and cells expressing non-functional NPC1 were tested for an increase in choles- 
terol release via exosomes. To test the specificity of this process, we transfected wild-type NPC1 into NPC1 mutant cells to re-establish normal exosome secretion. 


\section{Chapter 2}

\section{Materials and Methods}

\subsection{Materials}

\subsubsection{Chemicals and consumables}

Unless stated otherwise, all chemicals used in this study were purchased from AppliChem GmbH (Darmstadt, Germany), Merck KGaA (Darmstadt, Germany) or Sigma-Aldrich Chemie GmbH (Munich, Germany). Cell culture media, supplements, antibiotics and sera were obtained from Invitrogen GmbH (Darmstadt, Germany) or PAA Laboratories GmbH (Pasching, Austria). Consumables used in cell culture, molecular biology and biochemistry analysis were purchased from Falcon (Becton Dickinson Labware Europe, Le Pont De Claix, France), Eppendorf AG (Hamburg, Germany) or Greiner Bio-One GmbH (Frickenhausen, Germany).

\subsubsection{Cell lines and primary cells}

\subsubsection{Cell lines}

Cell lines used in this study are listed in Table 2.1.

Table 2.1: Cell lines

\begin{tabular}{lll}
\hline Name & Cell type & Obtained from \\
\hline \hline Oli-neu & Mouse oligodendrocyte precursor cells & J. Trotter, University of Mainz, Germany \\
CHO CT43 & Chinese hamster ovary cells & T. Y. Chang, Dartmouth Medical School, \\
& expressing non-functional, truncated NPC1 & Hanover, NH, USA \\
CHO K1 & Chinese hamster ovary cells & T. Y. Chang, Dartmouth Medical School, \\
& expressing wild-type NPC1 & Hanover, NH, USA \\
\hline
\end{tabular}




\subsubsection{Primary cells}

\section{Human skin fibroblasts}

Primary human skin fibroblasts from a healthy volunteer and a patient showing clinical symptoms of NPC disease (NPC-db patient-ID No. 158) were kindly provided by Heiko Runz from the Department of Human Genetics, Heidelberg, Germany. Material derived from the NPC patient is compound heterozygous for the mutations p.P1007A (c.3019C $>$ G) in exon 20 and p.R934X (c.2800C > T) in exon 19 of the npc1 gene.

\subsubsection{Antibodies}

Primary antibodies were used as listed in Table 2.2. Secondary fluorophore- or horse radish peroxidase (HRP)-conjugated antibodies were obtained from Dianova (Hamburg, Germany), Invitrogen GmbH (Darmstadt, Germany) or GE Healthcare (Munich, Germany).

Table 2.2: Primary antibodies

\begin{tabular}{llll}
\hline Target & Host species & Application & Obtained from \\
\hline \hline$\gamma$-Adaptin & Mouse & WB $(1: 5000)$ & BD Biosciences, Heidelberg, Germany \\
Alix/AIP & Mouse & WB $(1: 1000)$ & BD Biosciences, Heidelberg, Germany \\
Calnexin & Rabbit & WB $(1: 2000)$ & Stressgen Bioreag., Victoria, Canada \\
Flotillin-2 & Mouse & IF $(1: 100)$, WB $(1: 1000)$ & BD Biosciences, Heidelberg, Germany \\
GFP & Rabbit & WB $(1: 1000)$ & Abcam plc, Cambridge, UK \\
LAMP-1 & Rat & IF $(1: 50)$ & BD Biosciences, Heidelberg, Germany \\
Myc & Mouse & WB $(1: 1000)$ & Cell Signaling, Danvers, MA, USA \\
TSG-101 & Mouse & WB $(1: 1000)$ & GeneTex Inc., Irvine, CA, USA \\
\hline
\end{tabular}

\subsubsection{Nucleotide constructs}

\subsubsection{Plasmids}

\section{Previously published plasmids}

Plasmids obtained from other laboratories are listed in Table 2.3. 
Table 2.3: Published plasmids

\begin{tabular}{ll}
\hline Plasmid & Obtained from \\
\hline \hline EGFP-CD63 & D. Cutler, University College, London, UK \\
Flotillin-2-GFP & L. Rajendran, MPI of Molecular Cell Biology and Genetics, Dresden, Germany \\
GFP-rab7 & M. Zerial, MPI of Molecular Cell Biology and Genetics, Dresden, Germany \\
GFP-rab11 & M. Zerial, MPI of Molecular Cell Biology and Genetics, Dresden, Germany \\
MLV Gag-GFP & Addgene plasmid 1813, W. Mothes, Yale University School of Medicine, USA \\
pEYFP-6xHis-wtNPC1 & M. Scott, Stanford University, Stanford, USA \\
pEYFP-6xHis-NPC1 P692S & M. Scott, Stanford University, Stanford, USA \\
pR4-PLP-myc & J. Trotter, Johannes Gutenberg University, Mainz, Germany \\
\hline
\end{tabular}

\section{Self constructed plasmids}

Flotillin-2 mutated at one or both cholesterol recognition/interaction amino acid (CRAC) motifs was generated by site directed mutagenesis to introduce the amino acid point mutations Y124G or Y163G and the double mutation Y124G/Y163G. Mutagenesis was conducted according to the manufacturer's protocol (Quick Change Site-Directed Mutagenesis Kit, Agilent Technologies, Waldbronn, Germany).

\subsubsection{SiRNA constructs}

To down-regulate expression of flotillin-2 or NPC1, siRNAs were used as listed in Table 2.4.

Table 2.4: siRNA constructs

\begin{tabular}{lll}
\hline Target & siRNA & Reference \\
\hline \hline Mouse flotillin-2 & GUUCAUGGCAGACACCAAdTdT & Qiagen GmbH, Hilden, Germany \\
Mouse NPC1 & NPC1 Mn NPC1-1 predisigned siRNA & Qiagen GmbH, Hilden, Germany \\
Negative control & Control siRNA No. 1 & Ambion, Austin, USA \\
\hline
\end{tabular}

\subsubsection{Modifiers of cellular cholesterol homeostasis}

Cholesterol homeostasis was modified by compounds listed in Table 2.5. 
Table 2.5: Cholesterol modifiers

\begin{tabular}{lll}
\hline Compound & effect & Obtained from \\
\hline \hline Cholesterol in ethanol & Loading of free cholesterol & Sigma-Aldrich Chemie GmbH, \\
& & Munich, Germany \\
Cholesterol/ & Loading of free cholesterol & Sigma-Aldrich Chemie GmbH, \\
methyl- $\beta$-cyclodextrin complex & & Munich, Germany \\
CP-113.818 & Inhibition of Acyl-CoA cholesteryl acyl & Pfizer Inc., New York, NY, \\
& transferase (ACAT) & USA \\
Methyl- $\beta$-cyclodextrin & Acute cholesterol depletion & Sigma-Aldrich Chemie GmbH, \\
& & Munich, Germany \\
Mevalonate & Maintainance of geranylation/farnesylation & Sigma-Aldrich Chemie GmbH, \\
& & Munich, Germany \\
Simvastatin & Inhibition of HMG-CoA-reductase & Merck KGaA, Darmstadt, \\
& & Germany \\
U18666A & Impairment of cholesterol trafficking & Biomol, Plymouth Meeting, \\
& & PA, USA \\
\hline
\end{tabular}

\subsubsection{Buffers and solutions}

\subsubsection{Phosphate buffered saline (PBS)}

PBS was prepared according to Sambrook et al., 2001 (250).

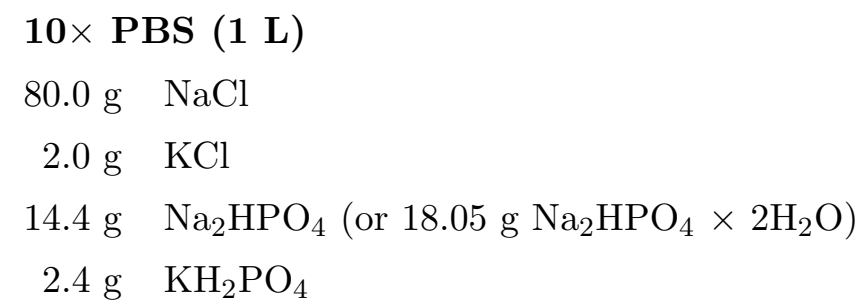

To obtain $1 \times$ PBS, $10 \times$ PBS was diluted 10 times with bi-distilled $\mathrm{H}_{2} \mathrm{O}$. The $\mathrm{pH}$ value was adjusted to 7.2-7.4.

\subsubsection{HEPES/sucrose stock solution}

HEPES/sucrose stock solution was prepared according to Théry et al., 2006 (27).

\section{$1 \times$ HEPES/sucrose $(1 \mathrm{~L})$}

$4.8 \mathrm{~g}$ Hydroxyethylpiperazine-N-2-ethanesulfonic acid (HEPES), $(\hat{=} 20 \mathrm{mM})$

$856 \mathrm{~g}$ Protease-free sucrose, $(\hat{=} 2.5 \mathrm{M})$

The $\mathrm{pH}$ was adjusted to 7.4 . 


\subsubsection{CHAPS lysis buffer}

\section{$1 \times$ lysis buffer $(1 \mathrm{~L})$}

$10 \mathrm{~g}$ 3-[(3-cholamidopropyl)dimethylammonio]-1-propanesulfonate (CHAPS), (^ 1\%)

$6.1 \mathrm{~g}$ Tris(hydroxymethyl)aminomethane (Tris), ( $\hat{=} 50 \mathrm{mM})$

$1.5 \mathrm{~g}$ Ethylenediaminetetraacetic acid (EDTA), $(\hat{=} 5 \mathrm{mM})$

The $\mathrm{pH}$ was adjusted to 8 .

\subsubsection{Protein loading buffer}

\section{$4 \times$ loading buffer}

$\begin{aligned} 10 \% & \text { Glycerol } \\ 50 \mathrm{mM} & \text { Tris-HCl } \\ 2 \mathrm{mM} & \text { EDTA } \\ 2 \% & \text { SDS } \\ 44 \mathrm{mM} & \beta \text {-mercaptoethanol } \\ 0.05 \% & \text { Bromophenol blue }\end{aligned}$

The $\mathrm{pH}$ was adjusted to 6.8 .

\subsubsection{Media and sera}

\subsubsection{Commercial media}

Table 2.6 depicts commercially available media, sera, and additives used in this study.

Table 2.6: Commercial media and solutions

\begin{tabular}{|c|c|}
\hline Medium/Solution & Obtained from \\
\hline Dulbecco's Modified Eagle Medium (DMEM) & PAA Laboratories GmbH, Pasching, Austria \\
\hline Fetal Calf Serum (FCS) & PAA Laboratories GmbH, Pasching, Austria \\
\hline Freestyle $^{T M} 293$ Expression Medium & Invitrogen GmbH, Darmstadt, Germany \\
\hline GlutaMAX ${ }^{\mathrm{TM}}$-I supplement & Invitrogen GmbH, Darmstadt, Germany \\
\hline Hanks' Balanced Salt Solution (HBSS) & Invitrogen GmbH, Darmstadt, Germany \\
\hline Horse Serum (HS) & PAA Laboratories GmbH, Pasching, Austria \\
\hline Insulin-Transferrin-Selenium - A supplement $100 \times$ & Invitrogen GmbH, Darmstadt, Germany \\
\hline LB medium + LB agar plate & AppliChem GmbH (Darmstadt, Germany) \\
\hline Lipoprotein Free Serum (LPS) & Sigma-Aldrich Chemie GmbH, Munich, Germany \\
\hline Opti-MEM + GlutaMAX ${ }^{T M}-I$ & Invitrogen GmbH, Darmstadt, Germany \\
\hline Phosphate Buffered Saline (PBS) & PAA Laboratories GmbH, Pasching, Austria \\
\hline Penicillin/Streptomycin (Pen/Strep) $100 \times$ & Invitrogen GmbH, Darmstadt, Germany \\
\hline
\end{tabular}




\subsubsection{SATO medium}

Sato medium was used for cultivation of the Oli-neu cell line. For preparation of serum free SATO, horse serum was omitted.

\section{SATO medium $(100 \mathrm{~mL})$}

$1 \mathrm{~mL}$ Insulin-Transferrin-Selenium-A Supplement ITS-A, 100×

$1 \mathrm{~mL}$ Putrescine dihydrochloride, stock $10 \mathrm{mM}$ in DMEM

$10 \mu \mathrm{L} \quad$ Progesterone, stock $2 \mathrm{mM}$ in ethanol

$10 \mu \mathrm{L}$ Triiodothyronine (Calbiochem/Merck KGaA, Darmstadt, Germany), $5 \mathrm{mM}$ stock in ethanol

$13 \mu \mathrm{L}$ L-Thyroxine (Calbiochem/Merck KGaA, Darmstadt, Germany), $4 \mathrm{mM}$ stock in $0.26 \mathrm{~N} \mathrm{NaOH}, 25 \%$ ethanol

$1 \mathrm{~mL} \quad$ GlutaMAX ${ }^{\mathrm{TM}}$-I supplement, $200 \mathrm{mM}$

$1 \mathrm{~mL}$ Penicillin/Streptomycin, $5000 \mathrm{U} / 5000 \mu \mathrm{g}$

$5 \mathrm{~mL}$ Horse serum

in Dulbecco's Modified Eagle Medium (DMEM) with $4.5 \mathrm{~g} / \mathrm{L}$ glucose.

\subsubsection{General growth medium}

General growth medium was used for cultivation of human fibroblasts and Chinese hamster ovary $(\mathrm{CHO})$ cells. For preparation of serum free medium, no fetal calf serum was added.

\section{General growth medium $(100 \mathrm{~mL})$}

$1 \mathrm{~mL} \quad$ GlutaMAX ${ }^{\mathrm{TM}}$-I supplement, $200 \mathrm{mM}$

$1 \mathrm{~mL}$ Penicillin/Streptomycin, $5000 \mathrm{U} / 5000 \mu \mathrm{g}$

$10 \mathrm{~mL} \quad$ Fetal calf serum

in Dulbecco's Modified Eagle Medium (DMEM) with $4.5 \mathrm{~g} / \mathrm{L}$ glucose.

\subsubsection{Commercial kits}

Commercial kits used in this study are listed in Table 2.7. 
Table 2.7: Commercial kits

\begin{tabular}{lll}
\hline Kit & Application & Obtained from \\
\hline \hline Amaxa Basic Neucleofector Kit, & SiRNA nucleofection & Lonza, Basel, Switzerland \\
Primary Neurons & & \\
NucleoBond Xtra Midi Kit & DNA isolation & Machery-Nagel, Dueren, Germany \\
Quick Change Site-Directed & Point mutagenesis & Agilent Technologies, Waldbronn, \\
Mutagenesis Kit & & Germany \\
\hline
\end{tabular}

\subsubsection{Software}

Software was used as depicted in Table 2.8.

Table 2.8: Software

\begin{tabular}{lll}
\hline Software & Application & Source \\
\hline \hline ImageJ & Image processing and analysis & http://rsbweb.nih.gov/ij/ \\
LaTeX & Text processing & http://www.latex-project.org/ \\
Leica Confocal Software, 2.61 & Acquisition of confocal images & Leica Microsystems, Mannheim, Germany \\
Meta Imaging Series 6.1 & Image processing and analysis & Universal Imaging Corp., Downingtown, USA \\
MS Office Exel 2003 & Spreadsheet analysis & Microsoft GmbH, Berlin, Germany \\
STATISTICA 7 & Statistical analysis & StatSoft GmbH, Hamburg, Germany \\
\hline
\end{tabular}

\subsection{Methods}

\subsubsection{Molecular biology}

\subsubsection{Site-directed mutagenesis}

To introduce amino acid point mutations into the flotillin- 2 protein, mutagenesis was performed using Stratagene QuickChange ${ }^{T M}$ Site-Directed Mutagenesis Kit (see Table 2.7) according to the manufacturer's protocol. This method is based on site-specific mutagenesis by overlap extension principle as described in Papworth et al., 1996 (251). 


\subsubsection{Transformation of $E$. coli}

For amplification of plasmid DNA constructs in bacteria, the chemocompetent E. coli strand 'Subcloning Efficiency DH5 alpha' (see Table 2.1) was used. For transformation, $50 \mu \mathrm{L}$ of competent cells were thawed on ice for $5 \mathrm{~min}$. Thereafter, $100 \mathrm{ng}$ of the desired plasmid were added to the cells, the suspension was mixed and incubated on ice for $20 \mathrm{~min}$ followed by heat-shock at $42^{\circ} \mathrm{C}$ for 42 sec. After recovery on ice for $2 \mathrm{~min}, 500 \mu \mathrm{L}$ of $\mathrm{LB}$ medium ( $25 \mathrm{~g} \mathrm{LB}$ in $1 \mathrm{~L} \mathrm{H}_{2} \mathrm{O}$, autoclaved) was added and the cells were incubated for $1 \mathrm{~h}$ at $37^{\circ} \mathrm{C}$ with agitation. To select for single transformants, the culture was spread on LB plates (LB-Agar $40 \mathrm{~g}$ per $1 \mathrm{~L} \mathrm{H}_{2} 0$, autoclaved) supplemented with the appropriate antibiotics $(100 \mu \mathrm{g} / \mathrm{ml}$ ampicillin or $50 \mu \mathrm{g} / \mathrm{ml}$ kanamycin $)$ and incubated at $37^{\circ} \mathrm{Cfor} 12$ $20 \mathrm{~h}$.

\subsubsection{Plasmid DNA isolation from $E$. coli}

To amplify plasmid DNA from transformed E. coli at a medium-scale, a single colony was picked from the LB plate with an autoclaved tip and transferred to $200 \mathrm{~mL}$ of antibioticsupplemented LB medium. Bacteria were incubated for $10-16 \mathrm{~h}$ at $37^{\circ} \mathrm{Cwith}$ constant shaking. Thereafter, cells were harvested by centrifugation for $10 \mathrm{~min}$ at 3,000 rpm and $4^{\circ} \mathrm{C}$ and plasmid DNA was isolated and purified with the NucleoBond Xtra Midi Kit (see Table 2.7) according to the manufacturer's instructions. DNA was redissolved in autoclaved, bi-distilled $\mathrm{H}_{2} \mathrm{O}$.

\subsubsection{Determination of DNA concentration}

Concentrations of double-stranded DNA were determined by measuring the optical density at $260 \mathrm{~nm}\left(\mathrm{~A}_{260}\right)$ and applying the Beer-Lambert law (252). In the procedure, samples were diluted 100-fold with $\mathrm{H}_{2} \mathrm{O}$ and absorbance was read at $260 \mathrm{~nm}$ in UVette cuvettes (Eppendorf AG, Hamburg, Germany) with BioPhotometer plus (Eppendorf AG, Hamburg, Germany). For the calculation of DNA concentrations the following formula was used:

$$
\text { Concentration of DNA }(\mu \mathrm{g} / \mathrm{mL})=A_{260} \times \text { dilution factor } \times 50 \mu \mathrm{g} / \mathrm{mL}
$$

\subsubsection{Cell culture}

All cell culture work was carried out according to security level S1 safety rules. Work was conducted under sterile conditions, involving antiseptic cleaning of the equipment 
with $70 \%$ ethanol and sterile filtration of all media and solutions used with a $0.22 \mu \mathrm{m}$ polyethersulfone (PES) filter (Corning Inc., Corning, NY, USA).

\subsubsection{Cryoconservation of cells}

\section{Freezing of cells}

For long term storage of cell lines and human skin fibroblasts, cells were cultured on a $10 \mathrm{~cm}$ petri dish or a $75 \mathrm{~cm}^{2}$ cell culture flask, respectively, to $80 \%$ confluence. Cells were then washed of or trypsinised as decribed in Section 2.2.2.2. After centrifugation of the cell suspension for 5 min at $900 \mathrm{~g}$ the pellet was resuspended in $0.5 \mathrm{~mL}$ DMEM. Subsequently, $0.5 \mathrm{~mL}$ of $2 \times$ freezing medium (40\% FCS, $20 \%$ DMSO in DMEM) was

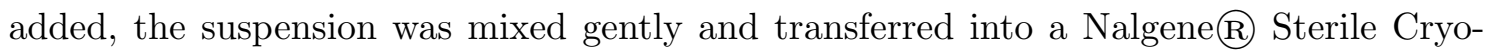
genic Vial (Thermo Fisher Scientific, Roskilde, Denmark). Cells were transferred to a Nalgene $\left(\right.$ Cryo $1{ }^{\circ} \mathrm{C}$ Freezing Container (Thermo Fisher Scientific, Roskilde, Denmark) and slow freezing at a temperature dropping rate of $-1{ }^{\circ} \mathrm{C} / \mathrm{min}$ was carried out in a $80^{\circ} \mathrm{C}$ freezer. For permanent storage, cells were stored in liquid nitrogen.

\section{Thawing of cells}

To thaw cryoconserved cells, a cryogenic vial was removed from liquid nitrogen and immediately incubated in a $37^{\circ} \mathrm{C}$ water bath. Immediately after the cell suspension became liquid, $10 \mathrm{~mL}$ of prewarmed fresh growth medium were added and the suspension was centrifuged for $5 \mathrm{~min}$ at $900 \mathrm{~g}$. The cell pellet was resuspended in prewarmed fresh growth medium and plated on a $10 \mathrm{~cm}$ petri dish or a $75 \mathrm{~cm}^{2}$ cell culture flask with previous PLL-coating, if necessary. Growing cells were further passaged according to cell type as described in Section 2.2.2.2.

\subsubsection{Growth and maintenance of cells}

In general, cells were grown at $37^{\circ} \mathrm{C}$ and $7.5 \% \mathrm{CO}_{2}$ in humidified incubators. Specific cultivation procedures are described below.

\section{Oli-neu cell line}

Cells of the oligodendrocyte precursor cell line Oli-neu were grown in SATO medium (see Section 2.1.7.2) on $10 \mathrm{~cm}$ plastic petri dishes previously coated with poly-L-lysine (PLL, $30 \mathrm{mg} / \mathrm{L}$ ) for $30 \mathrm{~min}$ and once washed with sterile water. Passaging of the cells was conducted 1:6 every 2-4 days after a confluence of 70-80 \% was reached. For passaging, cells 
were washed off with cultured medium to bring them in suspension. Cells up to passage number 30 were used for all experiments.

\section{Chinese hamster ovary cell lines}

The Chinese hamster ovary ( $\mathrm{CHO}$ ) cell lines CT43 and K1 were cultured in general growth medium (see Section 2.1.7.3) on $10 \mathrm{~cm}$ plastic petri dishes. Cells were passaged 1:10 every 3-5 days with a confluence of $80 \%$. For this, cells were washed once with PBS and trypsinized (0.05\% trypsin/EDTA; Lonza GmbH, Wuppertal, Germany) at $37^{\circ} \mathrm{C}$ for 1 min to detach them from the petri dish. Trypsination was stopped with fresh growth medium.

\section{Human skin fibroblasts}

Primary human skin fibroblasts were grown in general growth medium (see Section 2.1.7.3) in $75 \mathrm{~cm}^{2}$ cell culture flasks. Cells were passaged 1:4 every 3-4 days with a confluence of 80-90 \%. Fibroblasts were detached from the flask with $0.05 \%$ trypsin/EDTA. For collection of exosomes, cells were cultured in $10 \mathrm{~cm}$ plastic petri dishes in the absence of serum.

\subsubsection{Modification of cellular cholesterol}

Cholesterol loading and depletion treatments were conducted with Oli-neu cells plated on $6 \mathrm{~cm}$ plastic petri dishes (for Western blot experiments) or glass cover slips (for immunofluorescence staining).

\section{Cholesterol depletion}

Acute cholesterol depletion was achieved by washing the cells $3 \times$ with PBS followed by a $2 \mathrm{~h}$ incubation with $1 \mathrm{mM}$ methyl- $\beta$-cyclodextrin in Hanks' balanced salt solution (HBSS; see Table 2.6). Control cells were treated with HBSS only. After incubation time, cells were fixed for immunocytochemistry.

In another set of experiments, cholesterol depletion was achieved by inhibition of HMGCoA-reductase with simvastatin, which impairs the biosynthesis of cholesterol. For this, Oli-neu cells were washed $3 \times$ with PBS and pre-incubated for $24 \mathrm{~h}$ with horse serum (HS) free SATO medium supplemented with 1\% lipoprotein free serum (LPS; see Table 2.6). Following this, cells were incubated for $16 \mathrm{~h}$ (for Western blotting) or $48 \mathrm{~h}$ (for immunofluorescence) with $4 \mu \mathrm{M}$ simvastatin (in HS free SATO, 1\% LPS). Additionally, 
growth medium was supplemented with $50 \mu \mathrm{M}$ L-mevalonate to maintain normal geranylation and farnesylation. Control cells were incubated in HS free SATO, 1\% LPS, $50 \mu \mathrm{M}$ L-mevalonate. Immunofluorescence and Western blotting were performed after this treatment.

\section{Loading of free cholesterol}

Oli-neu cells were washed $3 \times$ with phosphate buffered saline (PBS; see Table 2.6 ) to remove all remainders of conditioned medium. Subsequently, cells were starved for $4 \mathrm{~h}$ in serum free SATO medium (see Section 2.1.7.2). To increase cellular cholesterol levels, cells were then incubated for $4 \mathrm{~h}$ in serum free SATO medium supplemented with free cholesterol at a final concentration of $50 \mu \mathrm{g} / \mathrm{ml}$ as methyl- $\beta$-cyclodextrin $(\mathrm{m} \beta \mathrm{CD})$ complex saturated with $10 \mu \mathrm{g} / \mathrm{ml}$ cholesterol dissolved in ethanol (final concentration of ethanol 0.1\%). Control cells were treated with $0.1 \%$ ethanol in serum free SATO medium. For immunofluorescence studies, cover slips were fixed after the incubation time. For Western blot assays, cells were lysed and the cultured medium was collected for exosome preparation (see Section 2.2.3.1).

\section{Inhibition of ACAT}

For inhibition of Acyl-CoA cholesteryl acyl transferase (ACAT), the inhibitor CP-113.818 was applied at a final concentration of $10 \mu \mathrm{M}$ in SATO medium for $24 \mathrm{~h}$ (CP-113.818 dissolved in DMSO, final concentration of DMSO 0.05\%). Subsequently, cells were washed with PBS $3 \times$ and further incubated in serum free SATO medium with $10 \mu \mathrm{M}$ CP-113.818 for $16 \mathrm{~h}$. Incubation medium of control cells lacked CP-113.818. After this treatment, immunofluorescence and Western blotting were conducted.

\section{Inhibition of cholesterol trafficking by U18666A}

Administration of the compound U18666A achieved an impairment of free cholesterol trafficking from late endosomes/lysosomes to the ER and Goli compartments. For this, $1.5 \mu \mathrm{g} / \mathrm{mL}$ U18666A in SATO was added to the cells for $12 \mathrm{~h}$. After $3 \times$ washing with PBS, cells were further incubated in serum free SATO with $1.5 \mu \mathrm{g} / \mathrm{mL}$ U18666A for another $4 \mathrm{~h}$ and examined for changes in exosome secretion by Western blotting. For analysis via immunocytochemistry, cells were treated with $1.5 \mu \mathrm{g} / \mathrm{mL}$ U18666A in SATO for $24 \mathrm{~h}$. 


\subsubsection{Collection of exosomes}

In general, exosomes from Oli-neu cells were collected in serum-free SATO medium to avoid any contamination with serum derived exosomes. The specific collection times, depending on treatment, are described in the previous section. Exsosomes from cells transfected with CRAC-mutated flotillin-2 were collected for $4 \mathrm{~h}$.

Exosomes derived from the Chinese hamster ovary cell lines CT43 and K1 were collected for $5 \mathrm{~h}$ in Freestyle ${ }^{\mathrm{TM}} 293$ Expression Medium (see Table 2.6).

Harvest of exosomes from human skin fibroblasts was preceded by a $24 \mathrm{~h}$ depletion period in FCS free general growth medium (see Section 2.1.7.3) supplemented with $1 \%$ LPS and another $24 \mathrm{~h}$ of cultivation in general growth medium containing $10 \%$ FCS to achieve high vesicular cholesterol accumulation. Subsequently, exosomes were collected in serum free general growth medium for $4 \mathrm{~h}$.

\subsubsection{Transfection of plasmids}

Plasmid DNA was introduced into mammalian cell lines via Mirus Bio TransITß-LT1 Transfection Reagent (Mirus Bio LLC, Madison, WI, USA). Upon transfection, the cells were grown to a confluence of $60 \%$. The transfection reagent and plasmid DNA were added to Opti-MEM (see Table 2.6), mixed slightly and incubated at room temperature for 30-45 min. According to cultivation in various vessels sizes, the specific protocols are listed below. After incubation, the mixture was added to cells dropwise, the cultivation vessel was shaken gently and the cells were kept under culture conditions for 8-12 h until further use.

\begin{tabular}{|c|c|c|c|}
\hline Culture vessel & 12-well plate & $6 \mathrm{~cm}$ dish & $10 \mathrm{~cm}$ dish \\
\hline Opti-MEM & $50 \mu \mathrm{L}$ & $300 \mu \mathrm{L}$ & $600 \mu \mathrm{L}$ \\
\hline TransIT & $1.5 \mu \mathrm{L}$ & $9 \mu \mathrm{L}$ & $18 \mu \mathrm{L}$ \\
\hline Plasmid DNA & $0.5 \mu \mathrm{g}$ & $3 \mu \mathrm{g}$ & $6 \mu \mathrm{g}$ \\
\hline
\end{tabular}

\subsubsection{RNA interference}

To down-regulate protein expression, siRNA was introduced into Oli-neu cells by electroporation with the Amaxa Basic Neucleofector Kit (see Table 2.7) in two steps. In a first step, cultured cells were suspended in fresh growth medium and $2 \times 10^{6}$ cells were centrifuged for $5 \mathrm{~min}$ at $900 \mathrm{~g}$. Pelleted cells were resuspended in $100 \mu \mathrm{L}$ Nucleofector solution provided with the kit and $160 \mathrm{pmol}$ of siRNA was added to the suspension. Using 
the program 'O-05' of the Amaxa Nucleofector (Lonza, Basel, Switzerland), siRNA was delivered into the cells. Immediately afterwards, the cells were plated on a PLL pre-coated $10 \mathrm{~cm}$ petri dish with warm, fresh SATO medium and were further cultured. In a second step 2 days thereafter, the cells were again collected and electroporated as described above. After resuspension in fresh SATO medium, cells were plated on a $6 \mathrm{~cm}$ petri dish at a density of $2 \times 10^{6}$ cells. The cells were used for further analysis after 2 days of cultivation.

\subsubsection{Exosome purification}

\subsubsection{Exosome preparation}

Conditioned growth medium from cultured cells secreting exosomes was collected as described in Section 2.2.2.4. To purify exosomes from the growth medium, a protocol of subsequent centrifugation steps adapted from Trajkovic et al., 2008 was applied (57). In the process, samples were handled on ice and all centrifugation steps were carried out at $4^{\circ} \mathrm{C}$ to avoid protein degradation. Remaining cells were cleared from the conditioned medium by centrifugation steps of $1 \times 3,000 \mathrm{~g}, 10 \mathrm{~min}$ and $2 \times 4,000 \mathrm{~g}, 10 \mathrm{~min}$. Subsequently, cell debris and larger microvesicles were pelleted in a step of 10,000 $\mathrm{g}$ for $30 \mathrm{~min}$. For final collection of exosomes in a pellet, the supernatant was centrifuged at 100,000 $\mathrm{g}$ for $1 \mathrm{~h}$. To normalize the amounts of exosome-released proteins and cholesterol, we also harvested corresponding cells, from which exosomes were collected. For protein analysis, corresponding cells of a $6 \mathrm{~cm}$ petri dish were scraped in $100 \mu \mathrm{L}$ of CHAPS lysis buffer (see Section 2.1.6.3) and centrifuged for $5 \mathrm{~min}$ at $5,000 \mathrm{rpm}$ and $4^{\circ} \mathrm{C}$. For cholesterol analysis, corresponding cells of a $10 \mathrm{~cm}$ petri dish were scraped in $800 \mu \mathrm{L}$ TE buffer. Pelleted exosomes and cell lysates were subjected to further analysis as described in the following sections.

\subsubsection{Sucrose gradient ultracentrifugation}

For further purification, the pelleted exosome material was loaded on top of a sucrose gradient. To avoid protein degradation, samples and sucrose solutions were handled on ice. The exosomal pellet from Section 2.2.3.1 was resuspended thoroughly in $500 \mu \mathrm{L}$ $0.25 \mathrm{M}$ sucrose solution (in $20 \mathrm{mM}$ HEPES, $\mathrm{pH} 7.4$ ) and the suspension was channeled $5 \times$ through a $26 \mathrm{G}$ needle to seperate single exosomes and avoid large membrane clusters. The suspension was then loaded on top a of discontinuous sucrose gradient consistent of 8 layers in total as depicted in Table 2.9, each layer with a volume of $500 \mu \mathrm{L}$. Ultracentrifugation 
of the samples using the swing out rotor SW60 Ti (Beckman Coulter GmbH, Krefeld, Germany) for $12 \mathrm{~h}$ at $200,000 \mathrm{~g}$ and $4^{\circ} \mathrm{C}$ sorted membrane vesicles along the sucrose gradient according to their density (see Table 2.9). After centrifugation, 8 fractions (each with a volume of $500 \mu \mathrm{L}$ ) were carefully taken from the top of the vial. Each layer was diluted 1:6 with PBS and again ultracentrifuged for $1 \mathrm{~h}$ at 100,000 $\mathrm{g}$ and $4^{\circ} \mathrm{C}$ to pellet the membrane material. Pellets were resuspended in $15 \mu \mathrm{L}$ protein loading buffer (see Section 2.2.4.2) and subjected to SDS-PAGE and Western blotting.

Table 2.9: Sucrose density gradient

\begin{tabular}{|c|c|c|c|}
\hline $\begin{array}{l}\text { Molarity of sucrose } \\
\text { (M) }\end{array}$ & $\begin{array}{l}\text { Corresponding density } \\
\left(\mathrm{g} / \mathrm{cm}^{3}\right)\end{array}$ & $\begin{array}{l}\text { Sucrose stock solution } \\
(2.5 \mathrm{M} \text { in } 20 \mathrm{mM} \text { HEPES) } \\
\text { for } 2.5 \mathrm{~mL}(\mathrm{~mL})\end{array}$ & $\begin{array}{l}20 \mathrm{mM} \text { HEPES } \\
\text { for } 2.5 \mathrm{~mL}(\mathrm{~mL})\end{array}$ \\
\hline 0.25 & 1.03 & 0.25 & 2.25 \\
\hline 0.57 & 1.07 & 0.57 & 1.93 \\
\hline 0.89 & 1.11 & 0.89 & 1.61 \\
\hline 1.21 & 1.16 & 1.21 & 1.29 \\
\hline 1.53 & 1.20 & 1.53 & 0.97 \\
\hline 1.86 & 1.24 & 1.86 & 0.64 \\
\hline 2.18 & 1.27 & 2.18 & 0.32 \\
\hline 2.50 & 1.32 & 2.5 & 0 \\
\hline
\end{tabular}

\subsubsection{Protein and lipid biochemistry}

\subsubsection{Determination of protein concentration}

To quantify protein concentrations, cell lysates were analyzed with Pierce BCA Protein Assay (Thermo Fisher Scientific Inc.,Rockford, IL, USA) using a detergent-compatible formulation based on bicinchoninic acid (BCA). The assay was conducted according to manufacturer's protocol and absorbance of BSA protein standards and experimental samples was measured at $562 \mathrm{~nm}$ wavelength. The concentration of the protein solution was calculated using the Beer-Lambert law (252):

$$
A=\epsilon \times l \times c
$$


A measured absorbance at a particular wavelength

$\epsilon$ molar absorption coefficient

$l$ path length

$c$ analyte concentration

\subsubsection{SDS-PAGE}

For separation of proteins according to their molecular weight under denaturating conditions, sodium dodecyl sulfate ployacrylamide gel electrophoresis (SDS-PAGE) was performed using the Bio-Rad Mini-PROTEAN 3 electrophoresis system (Bio-Rad Laboratories GmbH, Munich, Germany). Preparation of two layered polyacrylamide gels was performed in the Bio-Rad Mini-PROTEAN 3 casting system. Composition of the upper stacking gel $(2 \mathrm{ml})$ and the lower resolving gel $(5 \mathrm{ml})$ is specified below:

\section{Stacking gel}

$4 \%$ Acrylamide/bis-acrylamide (29:1) solution

$125 \mathrm{mM} \quad$ Tris- $\mathrm{HCl} \mathrm{pH} 6.8$

$0.1 \%$ SDS

$0.05 \%$ ammonium persulfate (APS)

$0.005 \%$ (v/v) N'N'N'-tetramethylethylene diamine (TEMED)

\section{Resolving gel (10 or $12 \%)$}

10 or $12 \%$ Acrylamide/bis-acrylamide (29:1) solution

$325 \mathrm{mM}$ Tris-HCl pH 6.8

$0.1 \%$ SDS

$0.05 \%$ APS

$0.005 \% \quad(\mathrm{v} / \mathrm{v})$ TEMED

For loading on the gel, exosome and cell lysate samples were prepared as follows: Exosome pellets were resuspended in denaturating protein loading buffer (see Section 2.1.6.4). $35 \mu \mathrm{L}$ of protein loading buffer were added to the cell lysate preparations. Before loading on the gel, the samples were boiled at $95^{\circ} \mathrm{C}$ for 5 min. Detection of PLP-myc protein required incubation at $55^{\circ} \mathrm{C}$ for 10 min to avoid multimer assembly. The gel was run in 
tris-glycine electrophoresis buffer (25 mM tris, $192 \mathrm{mM}$ glycine, 0.1\% SDS) for $90 \mathrm{~min}$ at 100 V. Loading of the marker PageRuler@ Plus Prestained Protein Ladder (Fermentas, St. Leon-Rot, Germany) enabled estimation of molecular weights of the analyzed proteins.

\subsubsection{Western blotting}

After separation via electrophoresis, proteins were subjected to Western blotting (253) using the Bio-Rad Mini-Protein System according to the manufacturer's protocol. In the procedure, proteins were transferred from SDS-polyacrylamide gels onto a Whatman@ Protran Nitrocellulose Transfer Membrane (Whatman GmbH, Dassel, Germany) by application of $100 \mathrm{~V}$ for $1 \mathrm{~h}$ at room temperature in transfer buffer (25 mM Tris, $192 \mathrm{mM}$ glycine, $20 \%$ methanol).

Following transfer of proteins, the nitrocellulose membrane was incubated in $4 \%$ nonfat dried milk (Sigma-Aldrich Chemie GmbH, Munich, Germany) in PBS for 30 min at room temperature to avoid nonspecific binding of immunoglobulins. The membrane was then incubated with primary antibody in PBST (0.1\% Tween-20 in PBS) (dilutions according to Table 2.2) for $8-12 \mathrm{~h}$ at $4^{\circ} \mathrm{C}$ followed by 3 washing steps of $10 \mathrm{~min}$ in PBST at room temperature. Subsequently, the membrane was incubated with horse radish peroxidase(HRP) conjugated secondary antibody (1:1,000 in PBST) for 1-2 $\mathrm{h}$ at room temperature and again washed $3 \times$ with PBST for $10 \mathrm{~min}$.

HRP-antibody binding was visualized by chemiluminescence using Pierce ECL Western Blotting Substrate (Thermo Fisher Scientific Inc.,Rockford, IL, USA). The emitted light signal was captured on X-ray films (CL-XPosure ${ }^{T M}$ Film, Thermo Fisher Scientific, Rockford, IL, USA), which were scanned with a conventional scanner and analyzed for light intensities with ImageJ (see Table 2.8).

\subsubsection{Cholesterol extraction and gas chromatography}

To extract cholesterol, exosome pellets were resuspended in $200 \mu \mathrm{L}$ TE buffer $(10 \mathrm{mM}$ Tris, $0.5 \mathrm{mM}$ EDTA, $\mathrm{pH} 8$ ). Corresponding parent cells of a $10 \mathrm{~cm}$ petri dish were washed $3 \times$ with PBS and scraped in $800 \mu \mathrm{L}$ TE buffer. From these, $200 \mu \mathrm{L}$ were taken for cholesterol extraction and the remains were stored at $-20^{\circ} \mathrm{C}$ for further analysis. The $200 \mu \mathrm{L}$ exosome and cell lysate samples were transferred into $2 \mathrm{ml}$ Eppendorf tubes and $5 \mu \mathrm{L}$ of lanosterol (100 ng/ $\mu \mathrm{L}$ in chloroform) as internal standard and $1 \mathrm{~mL}$ of methanol/chloroform mixture $(1: 2 \mathrm{v} / \mathrm{v})$ were added. The samples were incubated for $30 \mathrm{~min}$ at $4^{\circ} \mathrm{C}$ under 
constant shaking. After addition of $300 \mu \mathrm{L} \mathrm{H} \mathrm{H}_{2} \mathrm{O}$, the solutions were mixed and kept on ice for $15 \mathrm{~min}$ proceeded by centrifugation for $15 \mathrm{~min}$ at $1,300 \mathrm{rpm}$ and $4^{\circ} \mathrm{C}$. After phase separation, the lower organic phase was transferred to a glass vial, dried under streaming nitrogen and subjected to gas chromatography.

The analysis was performed with an Agilent 6890 gas chromatograph (Waldbronn, Germany) fitted with a capillary HP-5 column $(30 \mathrm{~m} \times 0.32 \mathrm{~mm}$; $0.25 \mu \mathrm{m}$ coating thickness; J\&W Scientific, Agilent). Helium was used as carrier gas $(1 \mathrm{ml} / \mathrm{min})$. The temperature gradient was $200^{\circ} \mathrm{C}$ for $1 \mathrm{~min}, 200-325^{\circ} \mathrm{C}$ at $20^{\circ} \mathrm{C} / \mathrm{min}$, and $325^{\circ} \mathrm{C}$ for $7.5 \mathrm{~min}$. Identity of cholesterol signal was verified with lanosterol as standard. Cholesterol contents of the samples were quantified by measuring the peak area of cholesterol and $500 \mathrm{ng}$ lanosterol.

(Gas chromatographic analysis of material was performed with the help of Cornelia Goebel.)

\subsubsection{Immunocytochemistry}

\subsubsection{Immunofluorescence staining}

Proteins were labeled with fluorescent antibodies via immunocytochemistry to determine their localization in cultured Oli-neu cells. All steps of this procedure were performed at room temperature. Oli-neu cells grown on PLL-coated glass cover slips were washed once with PBS and fixed in paraformaldehyde (4\% PFA in PBS, pH 7.4) for 15 min. After washing $3 \times$ with PBS, cells were permeabilized with $0.1 \%(\mathrm{v} / \mathrm{v})$ Triton X-100 (in PBS) for $1 \mathrm{~min}$ to enable antibodies to pass through the cell membrane. Cells were immediately washed $3 \times$ with PBS and covered with $100 \%$ blocking solution (see below) for 30 min to reduce unspecific binding of antibodies. Primary antibodies in $10 \%$ blocking solution were then incubated with the cells for $1 \mathrm{~h}$ in the concentrations indicated in Table 2.2. Preceded by 3 washing steps of 1 min with PBS, cells were incubated with fluorophore-conjugated secondary antibody in $10 \%$ blocking solution for another $45 \mathrm{~min}$ in the dark. After washing $3 \times$ with PBS for 1 min and once with bi-distilled $\mathrm{H}_{2} \mathrm{O}$ to remove salt remains, cells on cover slips were mounted onto glass slides with a drop of moviol solution (see below) and dried overnight. For long term storage, the prepared glass slides were kept in the dark at $4^{\circ} \mathrm{C}$.

Cells overexpressing proteins with a fluorescent tag (GFP or RFP) were fixed with PFA and directly mounted onto glass slides. 
$100 \%$ Blocking solution

$\begin{aligned} 2 \% & \text { BSA } \\ 2 \% & \text { horse serum } \\ 0.1 \% & \text { gelatine } \\ 0.1 \% & \text { saponin } \\ 0.02 \% & \text { biotin }\end{aligned}$

\section{Preparation of mowiol solution}

For preparation of mowiol mounting solution, $2.4 \mathrm{~g}$ mowiol (Calbiochem/Merck KGaA, Darmstadt, Germany) and $6 \mathrm{~g}$ glycerol were mixed with $6 \mathrm{~mL} \mathrm{H}_{2} \mathrm{O}$ and incubated at room temperature for $2 \mathrm{~h}$ with agitation. Subsequently, $12 \mathrm{~mL} 0.2 \mathrm{M}$ Tris/HCl (pH 8.5) were added and the mixture was heated for $10 \mathrm{~min}$ at $50^{\circ} \mathrm{C}$. Centrifugation at $5,000 \mathrm{~g}$ for 15 min facilitated clearance of the solution. Anti-fading reagent DABCO was added to a final concentration of $24 \mathrm{mg} / \mathrm{mL}$ and mowiol solution was aliquoted and stored at $-20^{\circ} \mathrm{C}$.

\subsubsection{Lysotracker staining}

Lysosomes of Oli-neu cells were labeled with the fluorescent acidotropic probe LysoTracker Red DND-99 (Invitrogen GmbH, Darmstadt, Germany). Cells were grown on cover slips, washed once and incubated in fresh SATO medium supplemented with $50 \mathrm{nM}$ LysoTracker for $1 \mathrm{~h}$ under growth conditions. Subsequently, the loading medium was replaced by fresh SATO medium and cells were imaged by fluorescence microscopy.

(Immunofluorescence and LysoTracker staining were partially performed by Sievert Weiss.)

\subsubsection{Filipin staining}

Free cholesterol in Oli-neu cells and human skin fibroblasts was labeled with filipin dye (Sigma-Aldrich Chemie GmbH, Munich, Germany). A freshly thawed aliquot of filipin was heated for $1 \mathrm{~h}$ at $37^{\circ} \mathrm{C}$ under constant shaking in the dark. The solution was then diluted 1:50 in PBS. PFA fixed and Triton permeabilized cells (prepared as in Section 2.2.5.1) were incubated with the diluted filipin solution for 15-20 min, washed $3 \times$ with PBS and once with bi-distilled $\mathrm{H}_{2} \mathrm{O}$ and mounted onto a glass slide. Samples were stored at $4^{\circ} \mathrm{C}$ protected from light.

(Filipin staining of human fibroblasts was performed by Heiko Runz.) 


\subsubsection{Microscopy}

\subsubsection{Confocal microscopy}

To image localization of immunostained or fluorescent protein-tagged proteins in fixed cell samples, confocal microscopy was conducted. Fluorescent images were acquired with a Leica DMIRE2 microscope with a $63 \times$ oil-immersion objective and a Leica TCS SP2 AOBS confocal laser scanning setup (Leica Microsystems, Mannheim, Germany).

(Microscopic analysis of cells was performed by Sievert Weiss.)

\subsubsection{Electron microscopy}

For acquisition of electron microscopic images, exosomes were prepared from cultured medium of Oli-neu cells as described in Section 2.2.3.1. Pelleted exosomes were then fixed in $4 \%$ paraformaldehyde and adsorbed to glow-discharged formvar-carbon-coated copper grids by floating the grids on $5 \mu \mathrm{L}$ droplets of the solution on parafilm for $10 \mathrm{~min}$. Samples were post-fixed with $1 \%$ glutaraldehyde for 2 min and negatively stained with $2 \%$ methylcelluloseuranyl acetate (containing $0.7 \mathrm{M}$ oxalate, $\mathrm{pH}$ 7). The grids were imaged with a LEO EM912 Omega electron microscope (Carl Zeiss, Jena, Germany). Digital micrographs were obtained with an on-axis $2048 \times 2048$ CCD camera (Proscan GmbH, Scheuring, Germany).

(Electron microscopic imaging of exosomes was performed by Wiebke Möbius.)

\subsubsection{Image processing and statistical analysis}

\subsubsection{Quantification of subcellular flotillin-2 distribution}

To quantify subcellular distribution of flotillin-2 protein, confocal images were taken with identical acquisition parameters and analyzed with Metamorph, Meta Imaging Series 6.1 software (see Table 2.8). The cell surface outer and inner borders were manually enframed using Metamorph software. Subsequently, fluorescence intensities of plasma membraneassociated and cytosolic flotillin-2 signal were determined (see Figure 2.1). Flotillin-2 distribution was calculated for 170 - 220 cells per experimental condition as the ratio of cytosolic versus total cellular (plasma membrane-associated + cytosolic) fluorescence in- 

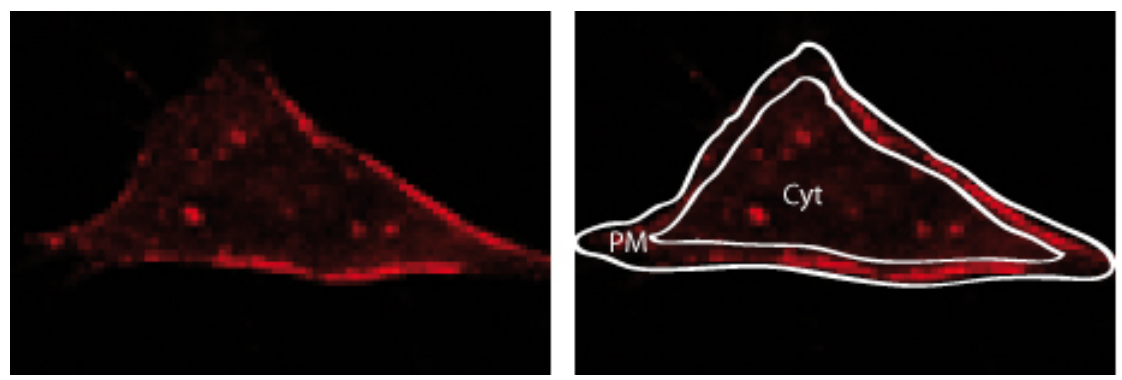

Figure 2.1: Quantification of subcellular flotillin-2 distribution. Oli-neu cells were imaged and analyzed with Metamorph software. The outer and inner borders of cell surfaces were manually enframed to measure signal intensities of plasma membrane-associated (PM) and cytosolic (Cyt) flotillin-2.

tensity.

(Endocytosis assays on immunofluorescence images were partially performed by Sievert Weiss.)

\subsubsection{Quantification of exosome secretion}

For comparison of relative exosome release between treatment groups, exosome pellets and corresponding parent cell lysates were subjected to Western blotting as described in Section 2.2.4.2 and Section 2.2.4.3. X-ray films of Western blot membranes were scanned and analyzed with ImageJ software for signal intensities of exosomal marker proteins (alix, flotillin-2 or EGFP-CD63) in exosome and cell lysate fractions. As a measure of exosome release, the ratio of signal intensities of exosomes versus corresponding cell lysates was calculated from 4 - 13 independent experiments.

\subsubsection{Statistical analysis}

Statistical analysis of data was performed with MS Office Exel 2003 (Microsoft Deutschland GmbH, Berlin, Germany) and STATISTICA 7 (StatSoft GmbH, Hamburg, Germany). For descriptive statistics, mean and standard error of the mean (SEM) of a data set were calculated and illustrated with MS Office Exel 2003. For analytical statistics, treatment groups were compared with STATISTICA 7. Data was analyzed for normality with the Kolmogorov-Smirnov test. To compare two independent groups with sample sets showing normal distribution and equal variance, the parametric Student's t-test was 
used. To detect differences between sample sets, which failed either normality or equal variance tests, the nonparametric Mann-Whitney U test was performed. Data groups with a p-value less than 0.05 were regarded as significantly different. 


\title{
Chapter 3
}

\section{Results}

Most of the results have been published in:

\author{
Exosome Secretion Ameliorates Lysosomal Storage of Cholesterol in Niemann- \\ Pick Type C Disease \\ Katrin Strauss, Cornelia Goebel, Heiko Runz, Wiebke Möbius, Sievert Weiss, \\ Ivo Feussner, Mikael Simons, and Anja Schneider \\ Journal of Biological Chemistry 285(34): 26279-26288 (2010)
}

\subsection{Cellular cholesterol levels regulate formation and release of exosomes}

\subsubsection{Cholesterol regulates the cellular distribution of flotillin-2}

In this study, we wanted to investigate whether exosomes can serve as molecular vehicles, which facilitate cholesterol efflux from cells to contribute to cholesterol homeostasis. If this was the case, exosome formation and release would have to be constantly adapted to cellular cholesterol levels. We therefore wanted to monitor both steps - generation and release of exosomes - under conditions were cholesterol levels were manipulated by administration of cholesterol lowering drugs or loading of cholesterol. For this purpose, we used the mouse oligodendroglial precursor cell line Oli-neu, which readily secretes exosomes in quantifiable amounts.

We first focused on exosome formation. The initial step of exosome generation involves invagination of the plasma membrane to form endosomes. A subset of those endosomes matures to late endosomes. Inward budding of the late endosomal membrane gives rise 
to MVBs, which are filled with intraluminal vesicles. The MVBs can either fuse with lysosomes for cargo degradation or they fuse with the plasma membrane to release their contents into the extracellular space. Released intraluminal vesicles are then termed exosomes.

The flotillin proteins have been described to associate with detergent resistant, cholesterolrich microdomains at the plasma membrane $(98 ; 117)$, and to govern a clathrin- and caveolin-independent endocytosis pathway (133). Flotillin-2 was also found to be enriched in exosomes (57), thus it can serve as a marker to follow the exosome pathway. We therefore studied whether flotillin-2 trafficking between the plasma membrane and endosomal vesicles is dependent on cellular cholesterol.

\subsubsection{Subcellular localization of flotillin-2}

Flotillin-2 was previously demonstrated to localize mainly to the plasma membrane (104; $254 ; 255)$, where it forms stable oligomer clusters (119). However, the distribution of flotillin-2 may vary for different cell types since it was also prominently found in several vesicular compartments inside kidney cells, T cells, and HeLa cells, including recycling endosomes, lysosomes and MVBs $(107 ; 108 ; 256)$. To determine the localization of flotillin-2 in Oli-neu cells under physiological conditions, we performed immunofluorescence analysis of cells with confocal microscopy.

Immunofluorescence staining of flotillin-2 revealed a predominantly vesicular localization. To define these vesicles in more detail, we transfected cells with GFP-tagged Rab-GTPases and stained for endogenous flotillin-2. Lysosomes were additionally labeled with lysotracker. Flotillin-2 only partially co-localized with GFP-Rab11, a marker for recycling endosomes (Figure 3.1A). It was predominantly found in late endosomes/lysosomes (GFPRab7, lysotracker, Figure 3.1B,C). In contrast, only a small portion of the protein was detected at the plasma membrane.

\subsubsection{Cholesterol depletion redistributes flotillin-2 to the plasma membrane}

To test whether subcellular flotillin-2 localization depends on cholesterol, we modulated cholesterol levels experimentally by administration of various drugs. We first analyzed flotillin-2 distribution in response to cholesterol depletion. This was achieved by acute withdrawal of cholesterol from the plasma membrane via methyl- $\beta$-cyclodextrin $(\mathrm{m} \beta \mathrm{CD})$. Incubation of Oli-neu cells with $1 \mathrm{mM} \mathrm{m} \beta \mathrm{CD}$ in HBSS for $2 \mathrm{~h}$ induced flotillin- 2 redistribution from intracellular, vesicular compartments to the plasma membrane, as seen in 

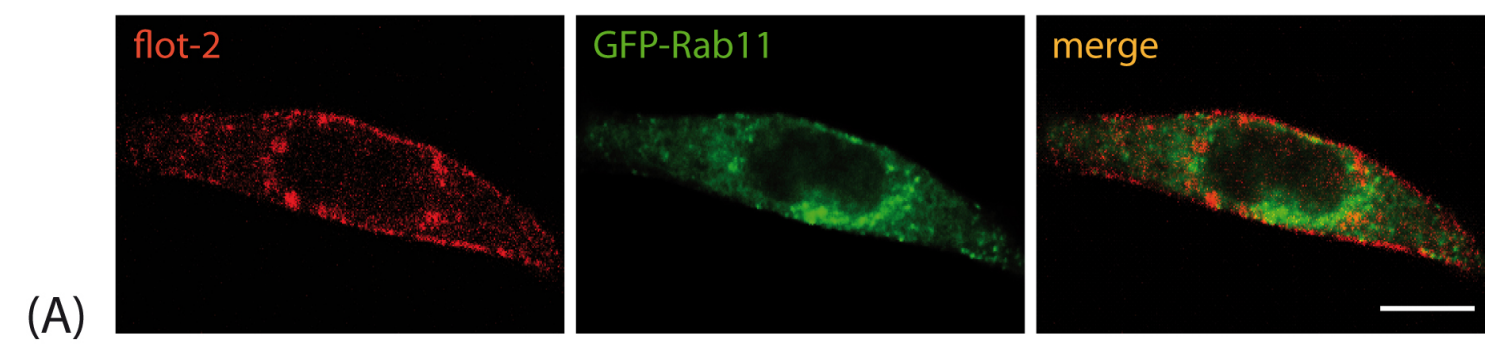

(B)
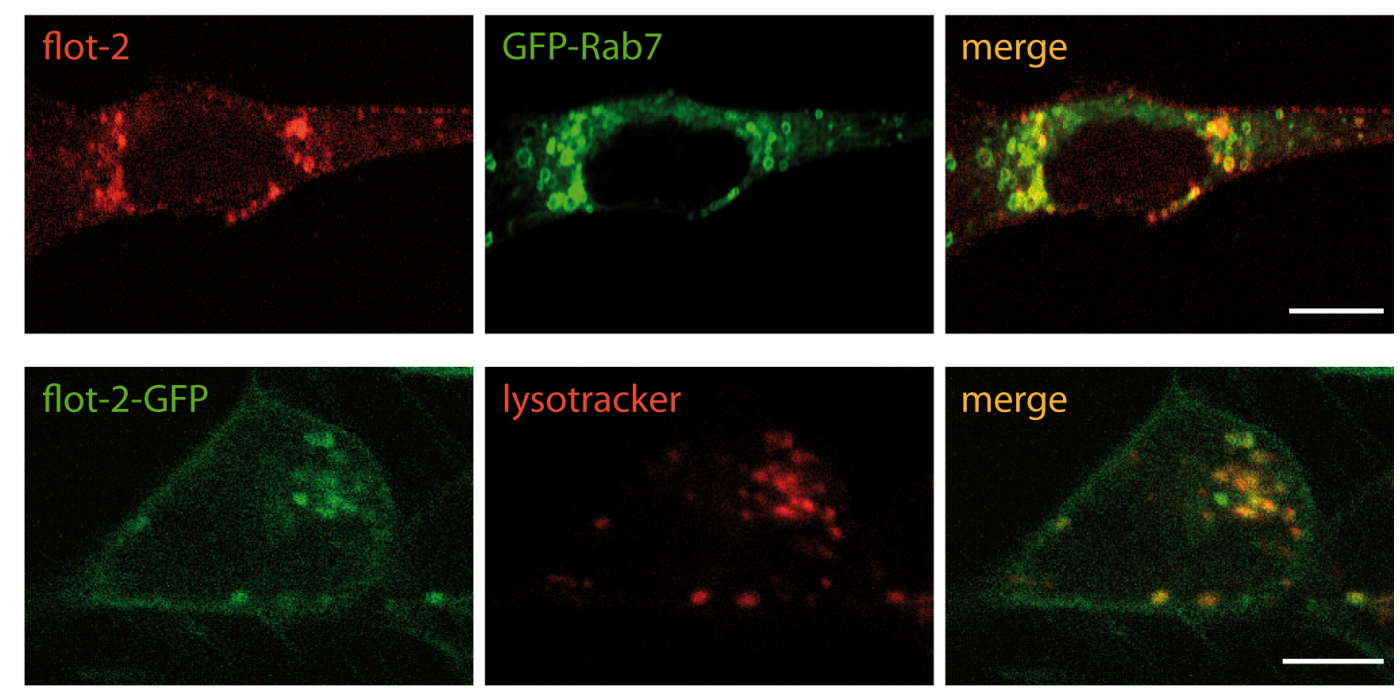

Figure 3.1: Flotillin-2 is localized to endosomes and lysosomes. Co-localization of flotillin-2 with endosomal and lysosomal markers was analyzed in Oli-neu cells. (A) Cells were transfected with GFP-Rab11 (green), a marker for recycling endosomes, fixed and endogenous flotillin-2 (flot2, red) was immunostained. Partial co-localization could be detected (merge). (B) Transfection with GFP-Rab7 (green), a late endosomal/lysosomal marker, and immunostaining of endogenous flotillin-2 (red) showed co-localization of both proteins (merge). (C) Exogenously expressed flotillin-2-GFP (flot-2-GFP, green) localized to lysosomes labeled with a fluorescent probe (lysotracker, red). Scale bars: $10 \mu \mathrm{m}$. 
immunofluorescence confocal microscopy images in Figure 3.2A (8.6\% shift from endosomal pools to plasma membrane, $\mathrm{SEM}=0.02, n=220$ cells, $\mathrm{p}=0.002$, Student's t test, 2 independent experiments).

Another way to decrease intracellular cholesterol is the incubation of cells with simvastatin. This drug inhibits HMG-CoA reductase, the rate limiting enzyme in cholesterol biosynthesis, catalizing L-mevalonate production. Since L-mevalonate is also essential for geranylation and farnesylation of numerous proteins, its reduction might cause an impairment of vital cellular functions. Therefore, to exclude any cholesterol-independent effects, cells were incubated for $48 \mathrm{~h}$ with $4 \mu \mathrm{M}$ simvastatin in SATO medium (1\% LPS) supplemented with $50 \mu \mathrm{M}$ L-mevalonate. Similarly to the observations under $\mathrm{m} \beta \mathrm{CD}$ treatment, inhibition of cholesterol synthesis by simvastatin accumulated flotillin- 2 at the plasma membrane and reduced its abundance at endosomal pools $(23.4 \%$ decrease of endosomal flotillin-2, SEM $=0.013, n=175$ cells, $\mathrm{p}<0.0001$, Student's t test, 2 independent experiments) (Figure 3.2B).

\subsubsection{Cholesterol loading accumulates flotillin-2 in endosomal pools}

With the previous results, we demonstrated that cholesterol depletion leads to flotillin-2 accumulation at the plasma membrane. In the next step, we wanted to know whether a rise in cholesterol level increases intracellular, vesicular flotillin-2 pools. Therefore, Oli-neu cells were starved for $4 \mathrm{~h}$ in serum free SATO medium and subsequently incubated for $4 \mathrm{~h}$ with $50 \mu \mathrm{g} / \mathrm{ml}$ cholesterol (as a methyl- $\beta$-cyclodextrin complex saturated with ethanoldissolved cholesterol). This treatment had no cytotoxic effects, as judged by the normal morphologic appearance of cells. In cholesterol loaded cells, a marked accumulation of cholesterol in cytoplasmic vesicles was identified by filipin staining as compared to the homogeneous cholesterol distribution in mock treated cells (Figure 3.3A).

To localize the accumulated cholesterol more precisely, cells were co-stained for various vesicular marker proteins. Filipin staining partially co-localized with the late endosomal marker protein GFP-Rab7, with the lysosomal marker LAMP-1, and with endogenous flotillin-2 and exogenously expressed EGFP-CD63, which are enriched in MVBs (Figure 3.3B). These results show that cholesterol and flotillin-2 localize in the same endosomal system.

We then analyzed the intracellular distribution of flotillin-2 after cholesterol loading. A pronounced redistribution of the protein to intracellular vesicles was observed $(13.6 \%$ increase, $\mathrm{SEM}=0.028, \mathrm{n}=171$ cells, $\mathrm{p}=0.0003$, Student's t test, 2 independent ex- 


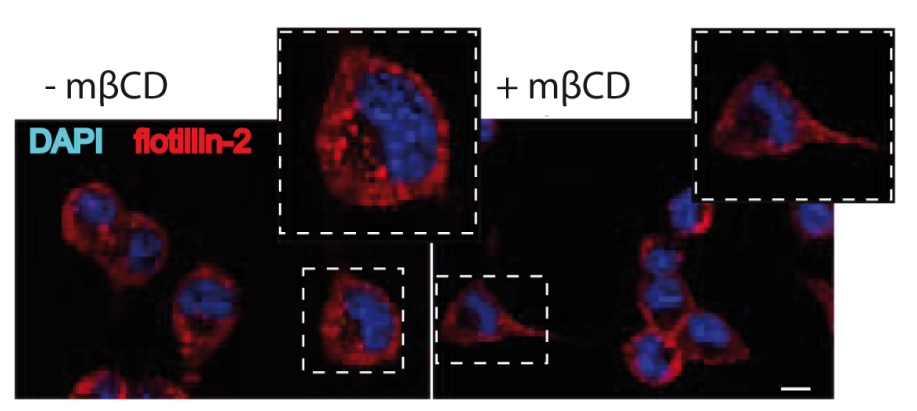

(A)

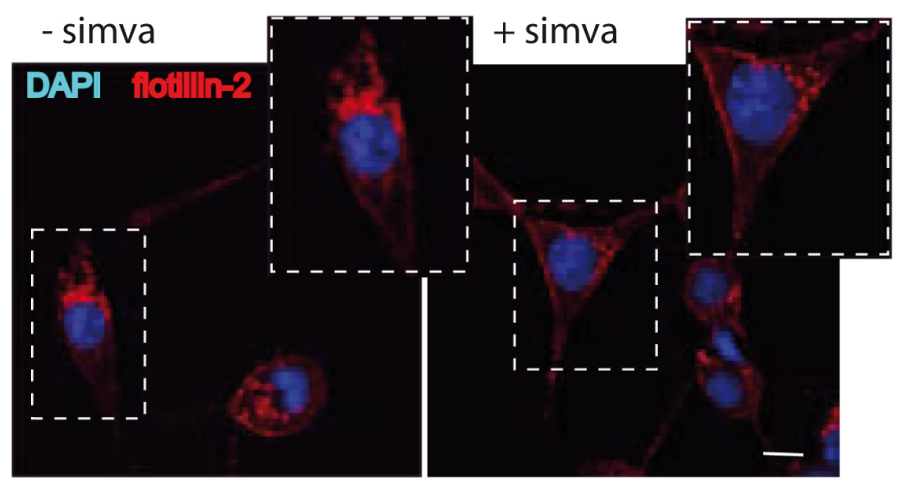

(B) vesicular/total flot-2

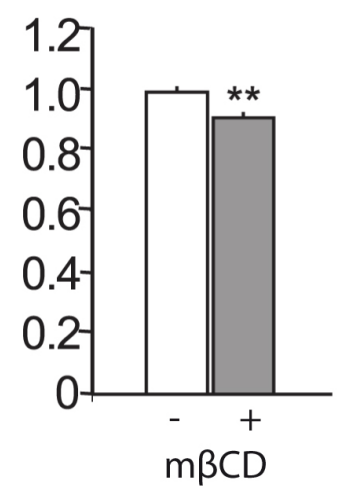

vesicular/total flot-2

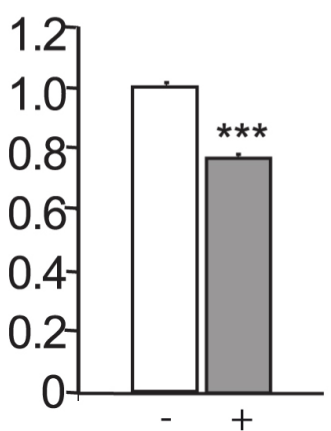

simva

Figure 3.2: Cholesterol depletion redistributes flotillin-2 to the plasma membrane. Oli-neu cells were chemically depleted from cholesterol (right) or mock treated (left). (A) $1 \mathrm{mM}$ methyl- $\beta$ cyclodextrin $(m \beta C D)$ was applied for $2 \mathrm{~h}$ to acutely withdraw cholesterol from cells. (B) Cells were incubated with $4 \mu \mathrm{M}$ simvastatin (simva) for $48 \mathrm{~h}$ to inhibit cholesterol biosynthesis. (A+B) After fixation, cells were immunostained for endogenous flotillin-2 (flot-2, red). The signal intensities of flotillin-2 were determined on confocal sections taken with identical acquisition parameters. Ratios of intracellular, vesicular versus total cellular flotillin-2 in response to treatment conditions are shown in histograms (right). Cholesterol depletion by $\mathrm{m} \beta \mathrm{CD}$ or simvastatin was accompanied by flotillin-2 redistribution from vesicular pools to the plasma membrane. Values are given as mean + SEM from 2 independent experiments. ${ }^{* *}$ indicates $p<0.005,{ }^{* * *} p<0.0005$. Scale bars: $10 \mu \mathrm{m}$. 
periments). In contrast, only moderate amounts of flotillin-2 located to vesicular compartments in control cells and parts of the protein were retained at the plasma membrane (Figure 3.3C).

\subsubsection{Intracellular cholesterol accumulation directs flotillin-2 to endosomal pools}

A second approach to increase cellular cholesterol levels is to trap the lipid in intracellular organelles, where they accumulate in large amounts. This can be achieved by the application of drugs, which interfere with intracellular cholesterol trafficking or metabolism.

U18666A is a compound known to inhibit cholesterol translocation from late endosomal/lysosomal compartments to the ER and the plasma membrane, resulting in cholesterol accumulation in late endosomes and lysosomes $(257 ; 231)$. Incubation of cells with $1.5 \mu \mathrm{g} / \mathrm{ml}$ U18666A for $16 \mathrm{~h}$ resulted in an increase of flotillin-2 localization at cytoplasmic vesicles compared to untreated controls $(7.06 \%$ increase, S.E. $=0.02, n=69$ cells, $p=$ 0.022 , Student's t test, 2 independent experiments) (Figure 3.4A). In these vesicles, the protein might be trapped together with free cholesterol.

Acyl-coenzyme A:cholesterol acyltransferase (ACAT) is an ER-resident enzyme catalyzing the conversion of free cholesterol into cholesteryl esters. When its activity is inhibited, cholesterol accumulates in the ER. We treated Oli-neu cells with $10 \mu \mathrm{M}$ ACAT inhibitor CP-113.818 for $24 \mathrm{~h}$. This increased the ratio of vesicular to total cellular flotllin-2 significantly $(21.8 \%$ increase, $\mathrm{SEM}=0.025, \mathrm{n}=213$ cells, $\mathrm{p}<0.0001$, Student's t test, 2 independent experiments) (Figure 3.4B).

In summary, all treatments decreasing or increasing cellular cholesterol levels had an impact on flotillin-2 distribution. While cholesterol depletion decreased incorporation of flotillin-2 into intracellular vesicular structures, high cholesterol levels enhanced its relocation from the plasma membrane to cytosolic vesicles. 

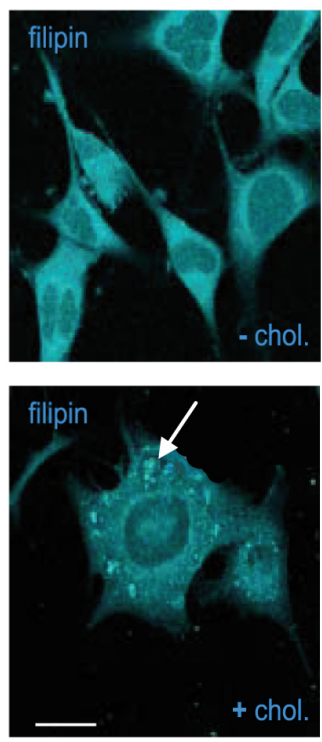

(A)

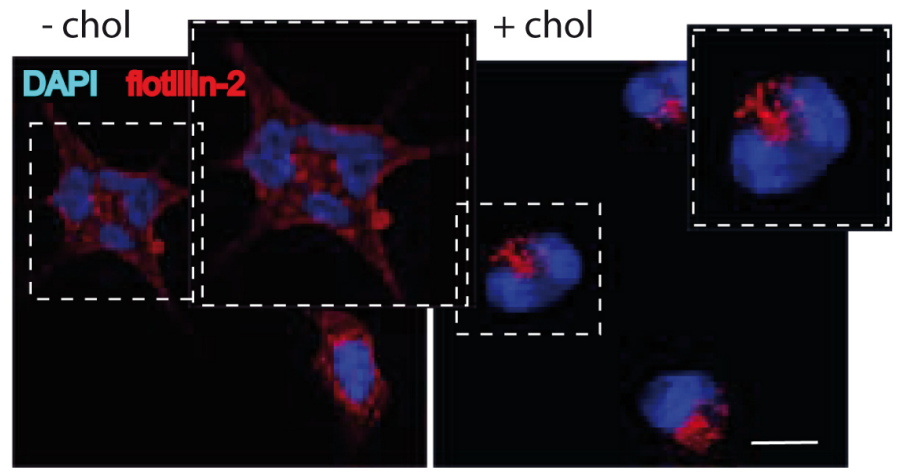

(C)
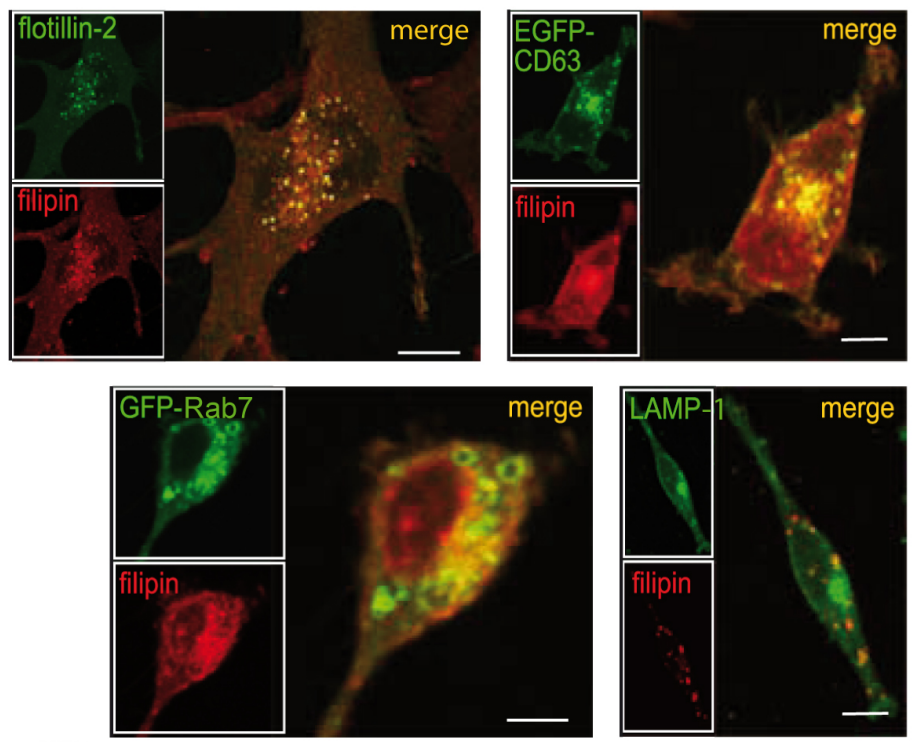

(B)

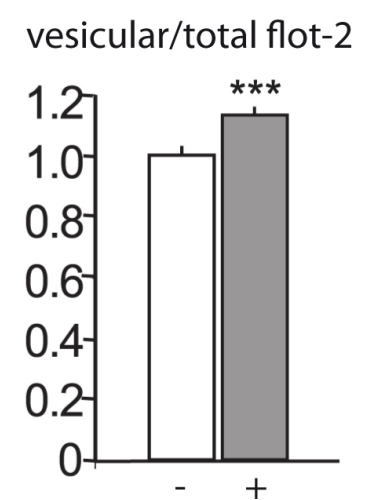

chol

Figure 3.3: Cholesterol loading accumulates flotillin-2 in endosomal pools. After starvation for $4 \mathrm{~h}$ in SATO medium supplemented with lipoprotein free serum, Oli-neu cells were treated with $50 \mu \mathrm{g} / \mathrm{ml}$ cholesterol in a saturated, water soluble complex for $4 \mathrm{~h}$ (+ chol) or not (- chol). (A) Staining cells with the fluorescent dye filipin showed vesicular accumulation of cholesterol (arrow) after cholesterol loading (bottom) in contrast to the homogeneous distribution in untreated controls (top). (B) Cells were loaded with cholesterol and co-stained for free cholesterol (filipin, pseudocolored in red) and markers (green) for multivesicular bodies/exosomes (flotillin-2, EGFP-CD63) or late endosomes/lysosomes (GFP-Rab7, LAMP-1). Cholesterol localized in these endocytic compartments. (C) Cholesterol and mock treated cells were fixed and immunostained for endogenous flotillin-2 (flot-2, red). The signal intensities of flotillin-2 were determined on confocal sections taken with identical acquisition parameters. Ratios of intracellular, vesicular versus total cellular flotillin-2 in response to treatment conditions are shown in the histogram. Flotillin-2 is shifted to intracellular compartments in cholesterol treated cells (gray bar). Values are given as mean + SEM from 2 independent experiments. ${ }^{* * *}$ indicates $\mathrm{p}<0.0005$. Scale bars: $10 \mu \mathrm{m}$. 

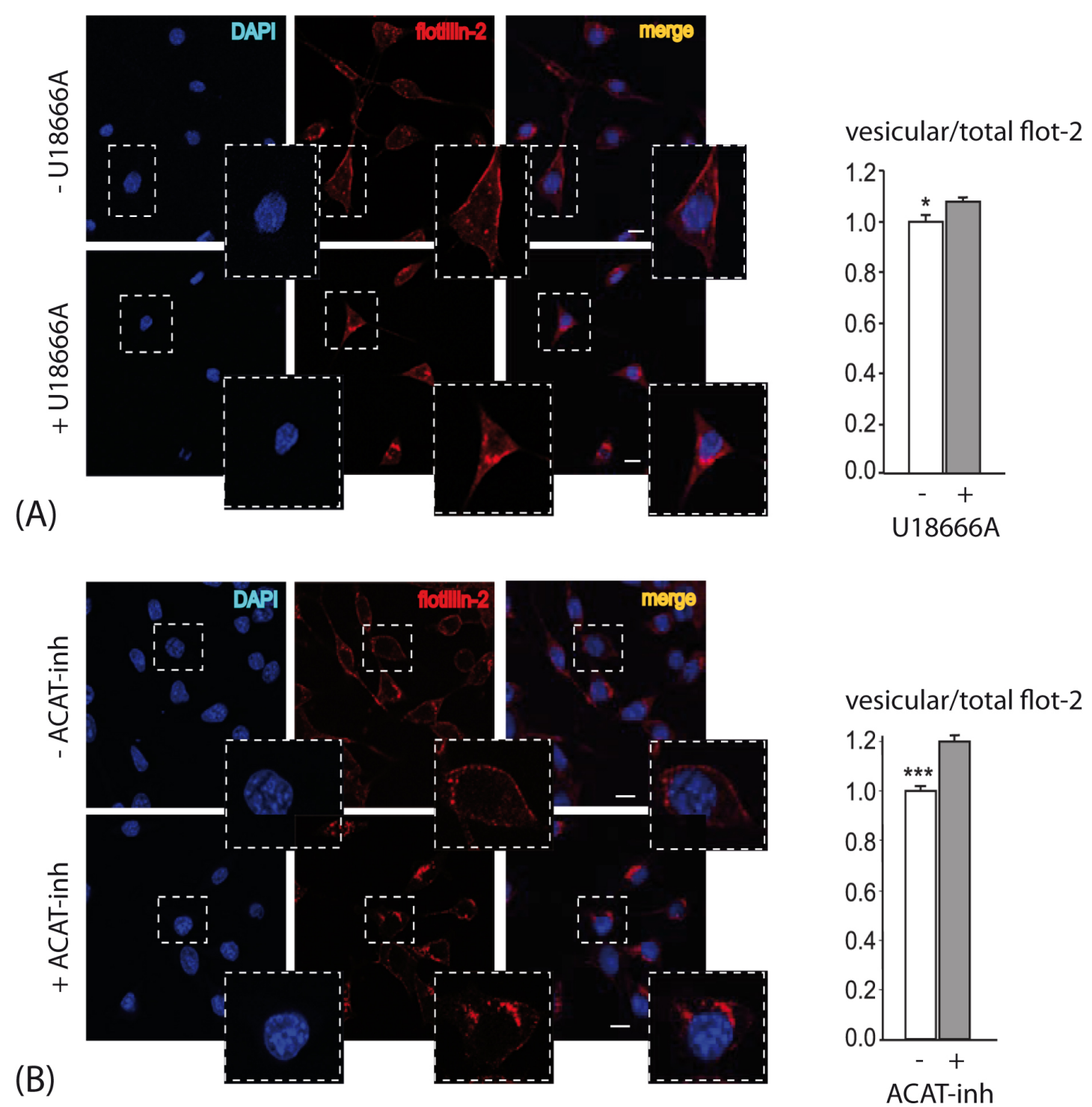

Figure 3.4: Intracellular trapping of cholesterol facilitates flotillin-2 accumulation in endosomal pools. Oli-neu cells were treated with chemicals promoting an intracellular accumulation of free cholesterol. (A) Treatment with $1.5 \mu \mathrm{g} / \mathrm{ml}$ U18666A in SATO medium for $24 \mathrm{~h}$ induced cholesterol accumulation in late endosomes/lysosomes. (B) The ACAT-inhibitor CP-113.818 (ACATinh) was applied to increase levels of free cholesterol in the ER. Cells were treated with $10 \mu \mathrm{M}$ CP-113.818 in SATO medium for $24 \mathrm{~h}$. (A+B) Fixed cells were immunostained for endogenous flotillin-2 (flot-2, red). The signal intensities of flotillin-2 were determined on confocal sections taken with identical acquisition parameters. Ratios of intracellular, vesicular versus total cellular flotillin-2 (flot-2) in response to treatment conditions are shown in the histograms (right). Flotillin-2 is shifted to intracellular compartments in treated cells (gray bars) compared to untreated control cells (white bars). Values are given as mean + SEM from 2 independent experiments. * indicates $\mathrm{p}<0.05,{ }^{* * *} \mathrm{p}<0.0005$. Scale bars: $10 \mu \mathrm{m}$. 


\subsubsection{Exosome release depends on cholesterol}

Having confirmed the cholesterol-dependence of flotillin-2 trafficking to endosomal compartments, we wanted to know whether cholesterol also influences the secretion of exosomes.

\subsubsection{Purification of exosomes}

To test the effect of cholesterol on exosome release, exosomes were prepared from culture medium of Oli-neu cells cultured under various treatment conditions. Cells were washed $3 \times$ with PBS and exosomes were collected in serum free SATO medium. This protocol prevented contamination of the samples with serum derived exosomes. For exosome preparation, the conditioned medium was cleared from dead cells and cell debris by a series of centrifugation steps (see Section 2.2.3.1). In a final centrifugation step of 100,000 $\mathrm{g}$ for $1 \mathrm{~h}$, exosomes were pelleted as previously described (57). We further refer to this $100,000 \mathrm{~g}$ pellet as the exosomal pellet (P100).

We validated the quality of this exosome preparation protocol by several tests:

Cell lysates from exosome secreting parent cells, as well as the pellets of each centrifugation step and the exosomal pellet were subjected to Western blotting and probed with flotillin-2 antibody. As presented in Figure 3.5A, flotillin-2 was enriched in the exosomal pellet.

Cell lysates and exosome pellets were further stained for other exosomal marker proteins. Besides for flotillin-2, we also found a robust signal for the two exosomal markers alix and tsg-101 (tumor susceptibility gene 101) in the 100,000 g pellets. A contamination of the $100,000 \mathrm{~g}$ pellet with membrane particles or vesicles other than exosomes could be excluded by the absence of staining for markers of distinct intracellular compartments, i.e. the ER marker calnexin and the trans-Golgi marker $\gamma$-adaptin (Figure 3.5B).

When the 100,000 $\mathrm{g}$ pellet was subjected to electron microscopy, we found the typical cup shaped structure of exsomes with a diameter between 50 and $100 \mathrm{~nm}$ (Figure 3.5C). No structures indicative of a contamination with larger microvesicles were detected. This additionally verifies the quality of our exosome preparations.

In another experiment to validate our exosome preparation protocol, sucrose gradient ultracentrifugation of the exosome pellet (P100) was performed. Hereby we wanted to analyze whether the material pelleted at 100,000 $g$ floats at exosome-characteristic densities. We allowed the material to distribute according to its densities in a sucrose gradient. After centrifugation at 200,000 $\mathrm{g}$ for $12 \mathrm{~h}, 8$ fractions were collected corresponding to densities 
flot-2

(A)

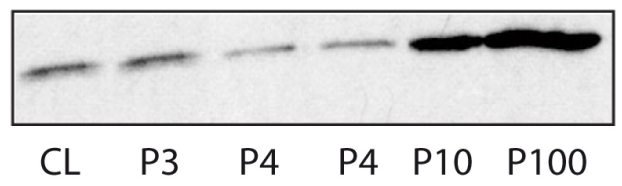

$\begin{array}{llllll}C L & P 3 & \text { P4 } & \text { P4 } & \text { P10 } & \text { P100 }\end{array}$

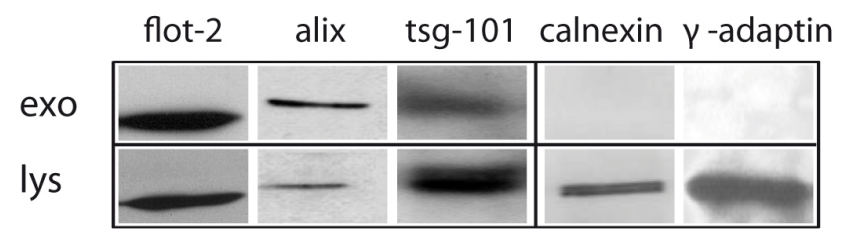

(B)

(C)

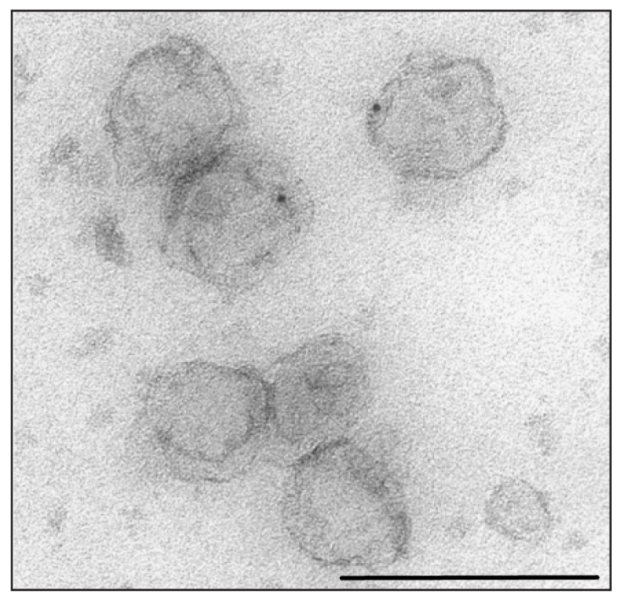

Figure 3.5: Exosomes can be purified by a sequence of centrifugation steps. (A) Cultured medium of Oli-neu cells was collected and subjected to a series of centrifugation steps to clear the medium from dead cells and cell debris with $1 \times 10 \mathrm{~min}$ at $3,000 \mathrm{~g}$ (P3), $2 \times 10 \mathrm{~min}$ at 4,000 $\mathrm{g}$ (P4), and $1 \times 30 \mathrm{~min}$ at 10,000 $\mathrm{g}$ (P10). Exosomes were collected in a final centrifugation step of $1 \mathrm{~h}$ at $100,000 \mathrm{~g}$ (P100). $20 \%$ of cell lysates (CL) and complete pellets were subjected to Western blot analysis. The exosome pellet (P100) is enriched in the exosomal marker protein flotillin-2 (flot-2). (B) Whole exosome pellets (exo) and $20 \%$ of corresponding cell lysates (lys) were immunostained in Western blot. Exosome fractions were positive for different exosomal marker proteins (flotillin-2, alix, tsg-101) but negative for markers of the ER (calnexin) and the Golgi apparatus ( $\gamma$-adaptin). (C) Electron microscopy of Oli-neu cell-derived exosomes showed their typical cup-like structure. Scale bar: $200 \mathrm{~nm}$. 


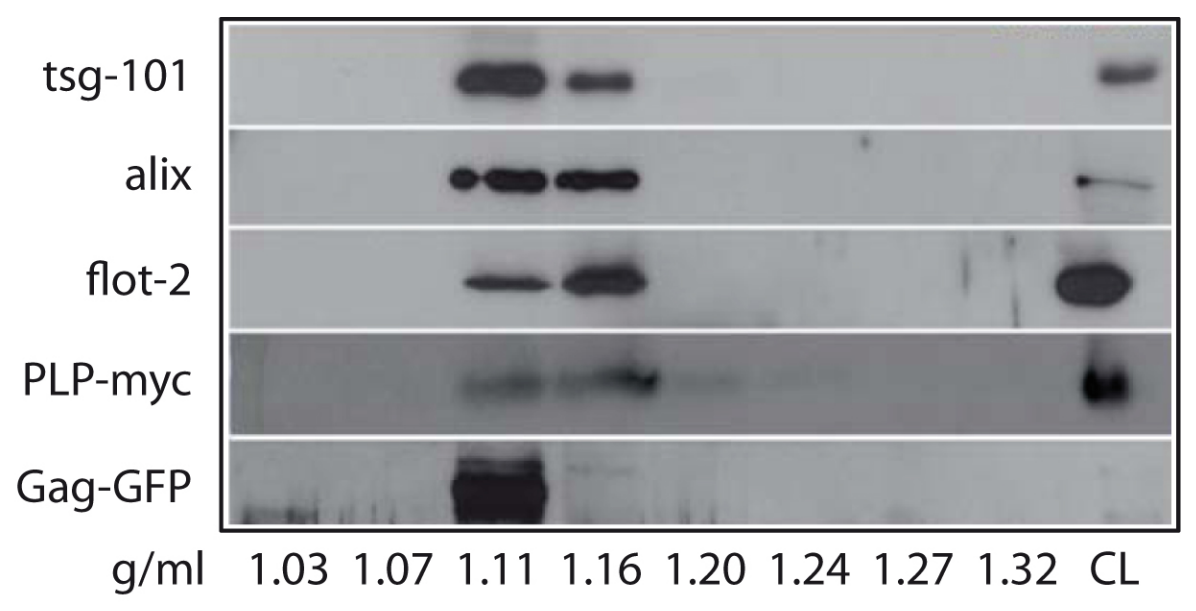

Figure 3.6: Sucrose gradient ultracentrifugation of the exosome pellet. For further purification, the exosome pellet was loaded on top of a sucrose gradient ranging from $0.25-2.5 \mathrm{M}$ (1.03$1.32 \mathrm{~g} / \mathrm{ml}$ ) and ultracentrifuged for $12 \mathrm{~h}$ at $200,000 \mathrm{~g}$ to equilibrium. Fractions were diluted $1: 6$ with PBS and re-centrifuged at 100,000 g. Pellets and cell lysates (CL) were analyzed by Western blotting for endogenous exosome marker proteins tsg-101, alix, and flotillin-2 (flot-2) and transiently expressed proteolipid protein (PLP-myc) and Moloney murine leukemia virus Gag protein (GagGFP). Detected signals correspond to the known exosome densities ranging from 1.11 to 1.18 $\mathrm{g} / \mathrm{ml}$.

between 1.03 and $1.32 \mathrm{~g} / \mathrm{ml}$. Fractions were subjected to immunoblotting and stained for exosomal marker proteins. Signals for flotillin-2, alix, and tsg-101 were detected in the fractions of 1.11 and $1.16 \mathrm{~g} / \mathrm{ml}$. This is in accordance with a flotation behavior described for exosomes $(27 ; 20)$. Transiently expressed PLP-myc (proteolipid protein) as well as Gag-GFP (Moloney murine leukemia virus Gag) were recovered from the same density fractions (Figure 3.6). PLP was shown to be enriched in exosomes derived from Oli-neu cells and primary oligodendrocytes $(258 ; 57 ; 21 ; 36)$. The Gag protein is resident in exosome-like vesicles, which immediately bud from the plasma membrane (56).

Taken these data together, we demonstrate that our exosome preparation protocol recovers material, which matches that obtained from other exosome purifications in size and density (20).

\subsubsection{Cellular cholesterol levels influence exosome release}

To determine whether cholesterol has an impact on exosome release, we quantified exosome secretion under drug induced changes in cholesterol levels. After treatment, we collected 


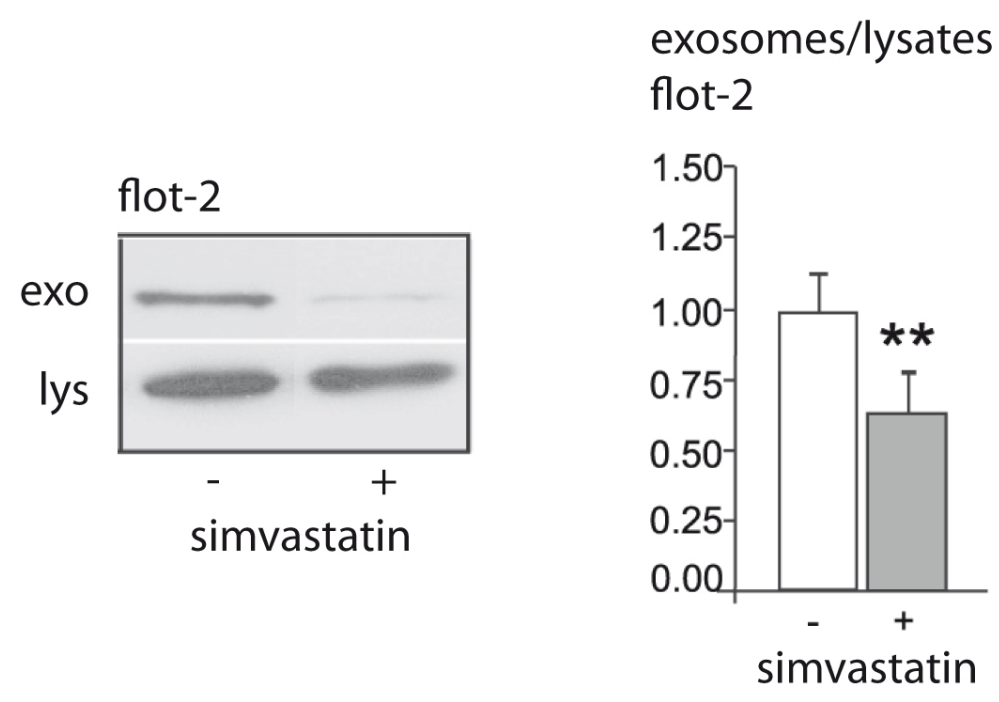

Figure 3.7: Cholesterol depletion decreases exosome release. Western blot and quantification of exosome release after treatment of Oli-neu cells with $4 \mu \mathrm{M}$ simvastatin for $16 \mathrm{~h}$ (gray bar) compared to untreated controls (white bar). Flotillin-2 immunostained blots were scanned and intensities of the bands were quantified. The ratio of endogenous flotillin-2 (flot-2) intensity in exosomal pellets (exo) versus cell lysates (lys) of exosome secreting parent cells was calculated. Values are given as mean + SEM from $n=12$ experiments. ${ }^{*}$ indicates $p<0.05$.

exosome pellets (P100) and parent cell lysates and subjected them to Western blotting. The samples were stained for the exosomal marker proteins flotillin-2, alix, and transiently expressed EGFP-CD63. For the quantification of exosome release, we determined the ratio of protein signal intensity in exosomes versus cell lysates.

We tested the effect of cholesterol depletion on exosome release by inhibiting cholesterol biosynthesis. Therefore, we incubated Oli-neu cells with $4 \mu \mathrm{M}$ simvastatin plus $50 \mu \mathrm{M}$ L-mevalonate for $16 \mathrm{~h}$. This treatment resulted in decreased secretion of exosomes as quantified by flotillin- 2 signals $(37.54 \%$ decrease, $\mathrm{SEM}=14 \%, \mathrm{n}=17, \mathrm{p}=0.0019$, MannWhitney U test) (Figure 3.7).

To rise cholesterol levels, cells were starved for $4 \mathrm{~h}$ in serum free SATO medium and subsequently incubated for $4 \mathrm{~h}$ with $50 \mu \mathrm{g} / \mathrm{ml}$ cholesterol/methyl- $\beta$-cyclodextrin complex (saturated with ethanol dissolved cholesterol). To ensure the consistency of our exosome preparation protocol along different treatment conditions, we performed sucrose gradient ultracentrifugation with exosome pellets of control cells and cells loaded with cholesterol. Treatment of cells did not change the flotation behavior of exosomal material at 1.11 and 


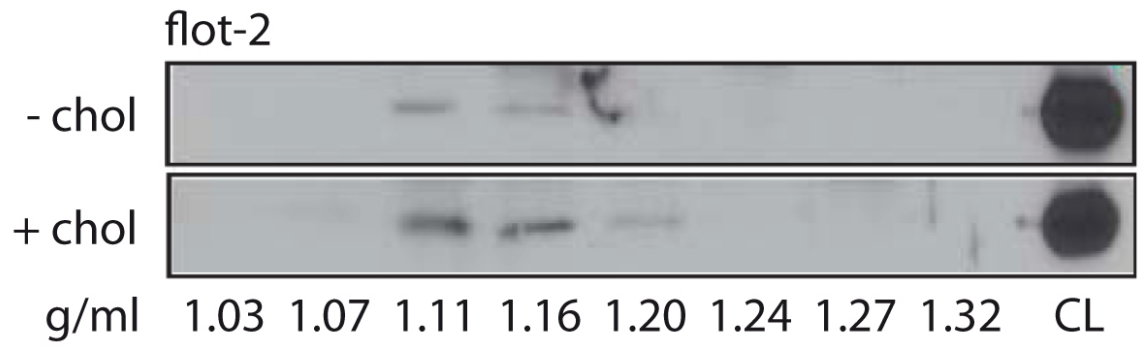

Figure 3.8: Sucrose gradient ultracentrifugation of exosome pellets derived from cholesterol loaded cells. Prior to exosome collection, cells were loaded with $50 \mu \mathrm{g} / \mathrm{ml}$ cholesterol in a saturated water soluble complex for $4 \mathrm{~h}(+\mathrm{chol})$ or not (-chol). Exosome pellets were subjected to sucrose gradient ultracentrifugation as described in Figure 3.6 and were subsequently analyzed by Western blotting with anti-flotillin-2 antibody. Flotillin-2 (flot-2) signal in exosome pellets of cholesterol treated cells was increased compared to controls. However, treatment had no effect on the type of released vesicles as shown by signals at the same densities in both, treatment and control group.

$1.16 \mathrm{~g} / \mathrm{ml}$ (Figure 3.8). This control also excluded cholesterol treatment-induced apoptosis, which would have led to a contamination of the exosome pellet with co-sedimented flotillin2-containing apoptotic vesicles floating at densities between $1.3-2 \mathrm{~g} / \mathrm{ml}$ (29). Loading of free cholesterol resulted in a markedly increased exosomal release of flotillin-2 (increase to $320 \%, \mathrm{SEM}=110 \%, \mathrm{n}=8, \mathrm{p}=0.022$, Mann-Whitney U test) (Figure 3.9).

This observation can be explained in two ways: Cholesterol could either only facilitate the incorporation of flotillin-2 into exosomes while the absolute number of secreted exosomes remains unchanged, or high cholesterol levels could increase exosome release per se. The latter assumption would imply an increase not only in the amount of flotillin-2 signal, but also in other exosomal marker proteins in our exosome preparation. To distinguish between both possibilities, Western blot membranes of exosomal pellets and cell lysates were further probed with antibodies against alix and CD63, two other exosomal markers. As shown in Figure 3.9, cholesterol loading also significantly enhanced exosomal release of endogenous alix and transiently expressed EGFP-CD63 (alix: increase to 2149.29\%, SEM $=1066.12 \%, \mathrm{n}=6, \mathrm{p}=0.004$, Mann-Whitney U test; EGFP-CD63: 628.30\% increase, $\mathrm{SEM}=93.77 \%, \mathrm{n}=4, \mathrm{p}=0.021$, Mann-Whitney $\mathrm{U}$ test). A cholesterol induced increase in the secretion of three different exosomal proteins rather indicates that the total number of released exosomes is up-regulated by high cholesterol levels. 

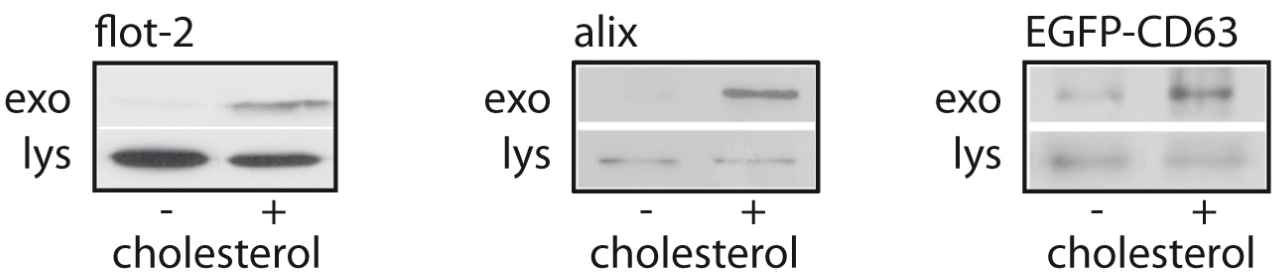

\section{exosomes/lysates}
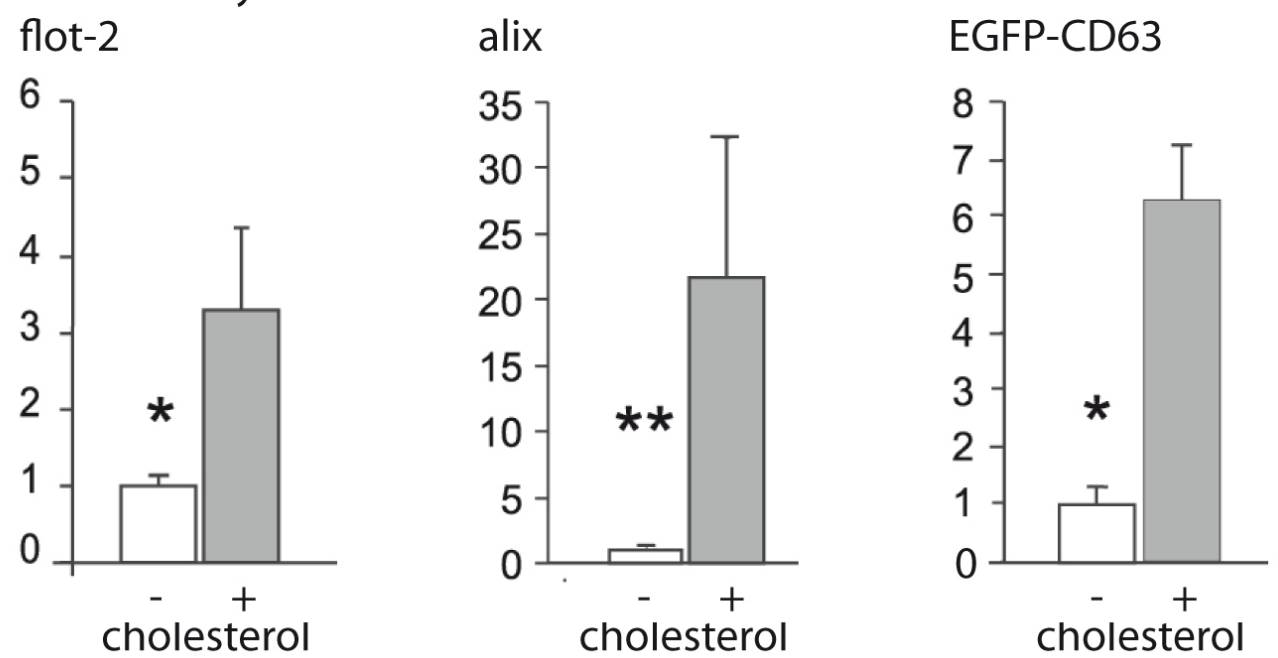

Figure 3.9: Loading of free cholesterol increases exosome release. After starvation for $4 \mathrm{~h}$ in SATO medium supplemented with lipoprotein free serum, Oli-neu cells were treated with $50 \mu \mathrm{g} / \mathrm{ml}$ cholesterol in a saturated, water soluble complex for $4 \mathrm{~h}$ (+ chol) or not (- chol). Exosome pellets (exo) and corresponding cell lysates (lys) were analyzed by Western blotting (top) for endogenous exosome marker proteins flotillin-2 (flot-2) and alix, and for the transiently expressed exosome marker EGFP-CD63. For quantification of exosome release, the ratio of protein intensity in the exosomal fraction versus protein intensity of the cell lysate was determined (bottom). For all three proteins immunoreactivity was increased in the exosome fraction after cholesterol treatment. Values are given as mean + SEM from $n=8,6$, and 4 experiments. * indicates $p<0.05$, ** $p<0.005$. 


\subsubsection{The delayed exosome pathway depends on cholesterol}

Exosomes are released in a delayed pathway via fusion of MVBs with the plasma membrane. There are however vesicles, which immediately bud from endosome-like domains of the plasma membrane and which resemble 'classical' exosomes in size, density and morphology (30). The proteins flotillin-2, alix, tsg-101, and transiently expressed PLP-myc are incorporated into exosomes derived from the endosomal system, i.e. from MVBs fusing with the plasma membrane. The protein Gag is known to be released via the immediate way of vesicle budding from the plasma membrane (56).

To distinguish between the delayed and the immediate pathway of exosome secretion, Oli-neu cells were transiently transfected to express PLP-myc or Gag-GFP. Cells were subsequently immunostained for these proteins and flotillin-2. The transmembrane protein PLP-myc was found at the plasma membrane and in intracellular vesicles, where it strongly co-localized with flotillin-2 (Figure 3.10A). In contrast, the protein Gag-GFP solely resided at the plasma membrane (Figure 3.10A and (56)). Only very rarely we detected colocalization of flotillin-2 with Gag-GFP.

We then asked whether cholesterol has an impact on both ways of exosome release - the delayed and the immediate one. PLP-myc or Gag-GFP transfected cells were loaded with $50 \mu \mathrm{g} / \mathrm{ml}$ cholesterol for $4 \mathrm{~h}$. The signal intensity of both proteins was then measured in exosome pellets and cell lysates by Western blotting. We detected a significant increase in exosomal PLP-myc release after cholesterol loading compared to mock treated cells (increase to $526.8 \%, \mathrm{SEM}=223 \%, \mathrm{n}=5, \mathrm{p}=0.03$, Mann-Whitney $\mathrm{U}$ test). This was comparable to the effect of cholesterol on exosomal release of flotillin-2, alix, and EGFPCD63. On the other hand, Gag-GFP release was not affected by cholesterol levels $(36.4 \%$ increase, $\mathrm{SEM}=0.45 \%, \mathrm{n}=12, \mathrm{p}=0.27$, Mann-Whitney U test) (Figure 3.10B). These data point towards a regulatory effect of cellular cholesterol levels on the indirect, delayed exosome pathway. An immediate budding of exosome-like vesicles from the plasma membrane remains, however, unaffected by cholesterol. 

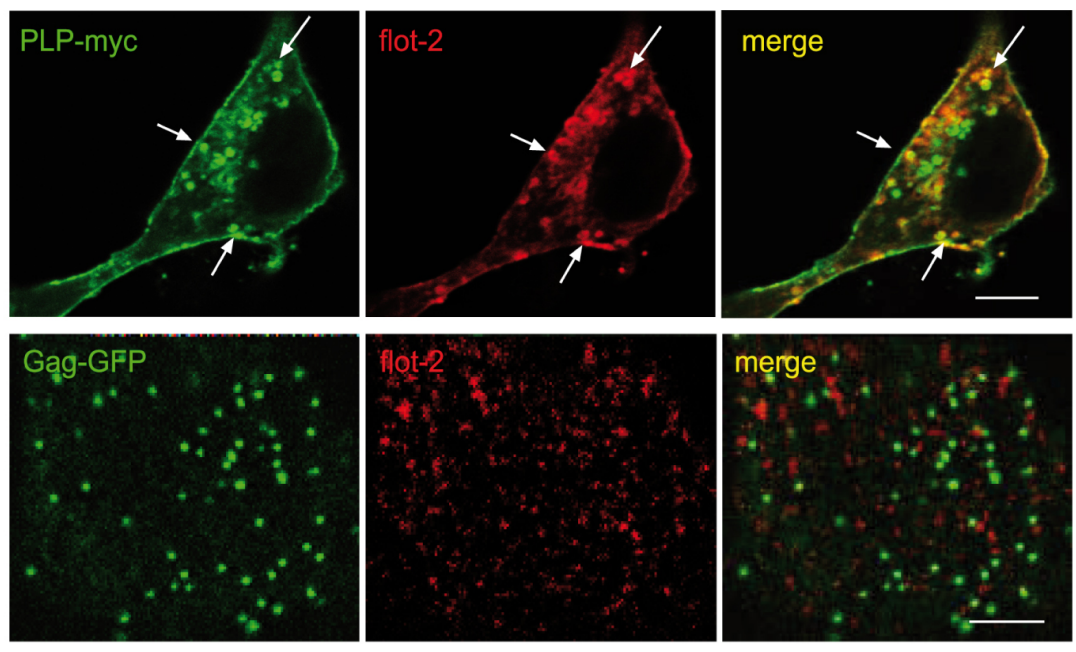

(A)

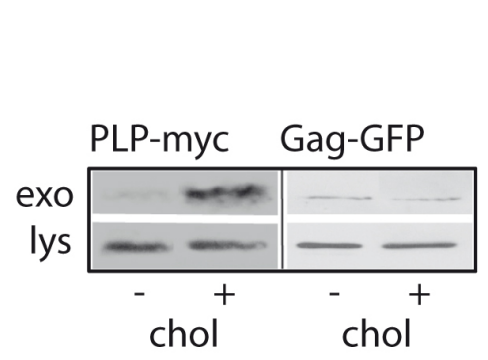

(B)

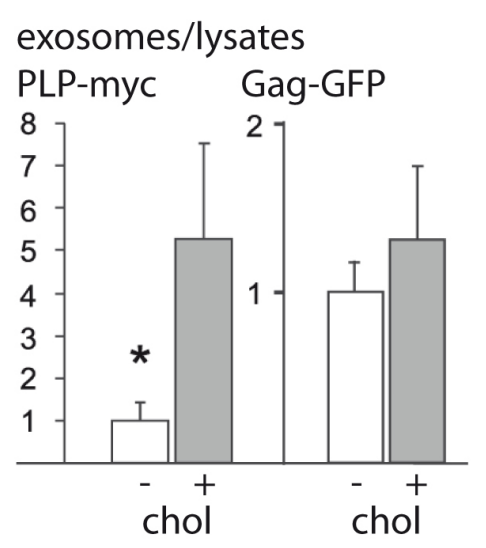

Figure 3.10: Cholesterol affects the delayed exosome pathway but not immediate vesicle shedding from the plasma membrane. Oli-neu cells were transfected for $12 \mathrm{~h}$ with PLP-myc, which is released by exosomes derived from endosomal pools, or with Gag-GFP, which directly buds from the plasma membrane. (A) Oli-neu cells were fixed, immunostained and analyzed for endogenous flotillin-2 (flot-2, red) and transiently expressed PLP-myc or Gag-GFP (green) by confocal microscopy. Flotillin-2 and PLP-myc co-localized in endosomal vesicular compartments (arrows, upper panel). In contrast, no co-localization of flotillin-2 and Gag-GFP could be detected (lower panal). Scale bars: $10 \mu \mathrm{m}$ (top) and $100 \mathrm{~nm}$ (bottom). (B) Preceded by starvation for $4 \mathrm{~h}$, Oli-neu cells were treated with $50 \mu \mathrm{g} / \mathrm{ml}$ cholesterol for $4 \mathrm{~h}$ (+ chol) or not (- chol). Signal intensities of the transiently expressed proteins PLP-myc and Gag-GFP were analyzed by Western blotting using anti-myc and anti-GFP antibodies. Blots were scanned, and the ratio of protein intensity in the exosomal fraction versus protein intensity in cell lysates of exosome secreting parent cells was determined. Exosomal release of PLP-myc was increased upon cholesterol treatment, whereas cholesterol had no significant impact on Gag-GFP release within exosome-like vesicles. Values are given as the mean + SEM from $n=5$, and 12 experiments. ${ }^{*}$ indicates $p<0.05$. 


\subsection{Flotillin-2 interacts with cholesterol and regulates its exosomal release}

Next, we were interested in the molecular mechanism, by which exosomal cholesterol release is mediated. Flotillin-2 is not only a marker protein for exosomes. It may also play an active role in the cholesterol-dependent formation of exosomes. This is indicated by two findings. The first is the observation that flotillins and cholesterol co-localize in the same endosomal compartments and membrane microdomains (our own results and $(98 ; 111)$ ). The second is the discovery of a novel flotillin-dependent pathway of endocytosis (133). It is therefore possible that flotillin-2 associates with cholesterol at the plasma membrane and drives the lipid into newly emerging endosomes, which mature within the endocytic pathway to intraluminal vesicle-containing MVBs that give rise to exosomes.

\subsubsection{Two cholesterol recognition/interaction amino acid consensus (CRAC) se- quences mediate subcellular distribution and exosomal release of flotillin-2}

We first wanted to examine, how flotillin-2 interacts with cholesterol. Two CRAC motifs were predicted for flotillin-2 (120). These 'cholesterol recognition/interaction amino acid consensus' sequences might enable the protein to bind to cholesterol and sort it at the plasma membrane. The CRAC domain was first discovered in the peripheral type benzodiazepine receptor (PBR), which transports cholesterol into mitochondria $(121 ; 122)$. It consists of a conserved amino acid sequence of (L/V-(X)(1-5)-Y-(X)(1-5)-R/K). When PBR's CRAC motif was mutated at the tyrosine $(\mathrm{Y})$ residue, uptake and translocation of cholesterol into mitochondria became impaired (123).

To verify the functionality of flotillin-2's CRAC domains, we introduced the same tyrosine point mutation into one or both CRAC domains spanning aa 120-127 (VEQIYQDR) and aa 157-169 (VYDKVDYLSSLGK) of flotillin-2-GFP. The resulting mutants were: flotillin-2-GFP Y124G, flotillin-2-GFP Y163G, and the double mutant flotillin-2-GFP Y124G/Y163G.

We then investigated whether mutation of the CRAC domains had an impact on subcellular localization of flotillin-2-GFP. Wild-type as well as CRAC mutated flotillin-2-GFP were transiently transfected into Oli-neu cells. The cells were subsequently fixed and analyzed by confocal microscopy. Only small amounts of wild-type flotillin-2-GFP were located at the plasma membrane, whereas a strong signal was found in cytoplasmic vesicular pools. In contrast, mutation of one or both CRAC domains led to an arrest of flotillin-2-GFP at 

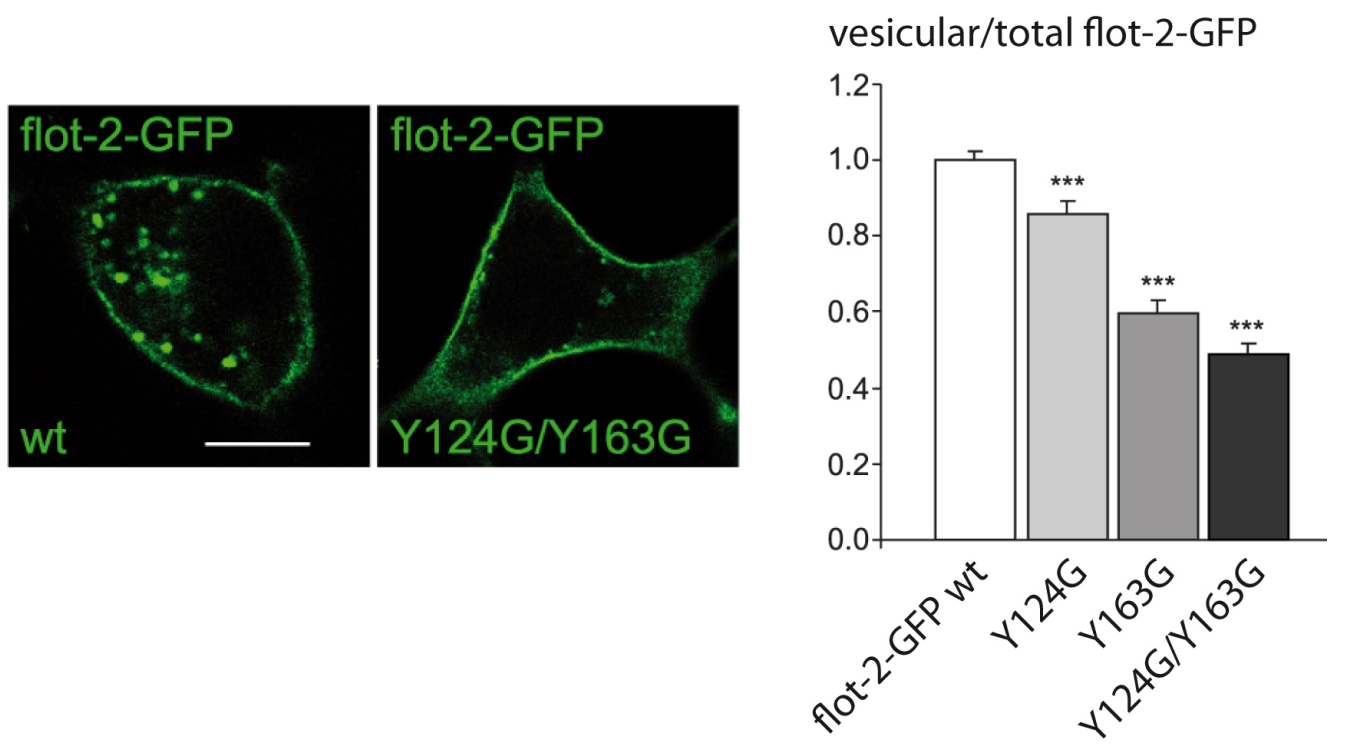

Figure 3.11: CRAC domains are required for intracellular vesicular localization of flotillin-2. Oli-neu cells were transfected with flotillin-2-GFP wild-type (flot-2-GFP wt) or flotillin-2-GFP bearing point mutations in one or two putative CRAC domains (Y124G, Y163G, and Y124G/Y163G). Cells were fixed and analyzed for immunofluorescence intensities of flotillin-2-GFP on confocal sections taken with identical acquisition parameters (left). Ratios of intracellular, vesicular versus total cellular flotillin-2-GPF are shown in the histogramm (right). While wild-type flotillin-2 was incorporated into vesicular structures, CRAC mutants accumulated at the plasma membrane. Note that the double mutant of both CRAC domains Y124G/Y163G (black bar) exerted the most pronounced effect. Values are given as mean + SEM from 3 independent experiments. ${ }^{* * *}$ indicates $\mathrm{p}<0.0005$. Scale bar: $10 \mu \mathrm{m}$.

the plasma membrane (Figure 3.11). This effect was strongest for flotillin-2-GFP Y124G and the double mutant flotillin-2-GFP Y124G/Y163G (51\% reduction, SEM $=0.05, \mathrm{p}<$ 0.0001, Student's t test, 3 independent experiments).

In a next step, we determined the influence of CRAC domains on the exosomal release of flotillin-2. We transfected Oli-neu cells with wild-type or mutant flotillin-2-GFP, and harvested exosomes and cell lysates, which were subjected to Western blotting. Mutation of both CRAC domains, Y124G and Y163G, resulted in a significant reduction of the protein's secretion via exosomes as compared to wild-type flotillin-2-GFP (54.48\% decrease, $\mathrm{SEM}=7.6 \%, \mathrm{n}=13, \mathrm{p}=0.0136$, Student's t test)(Figure 3.12). 


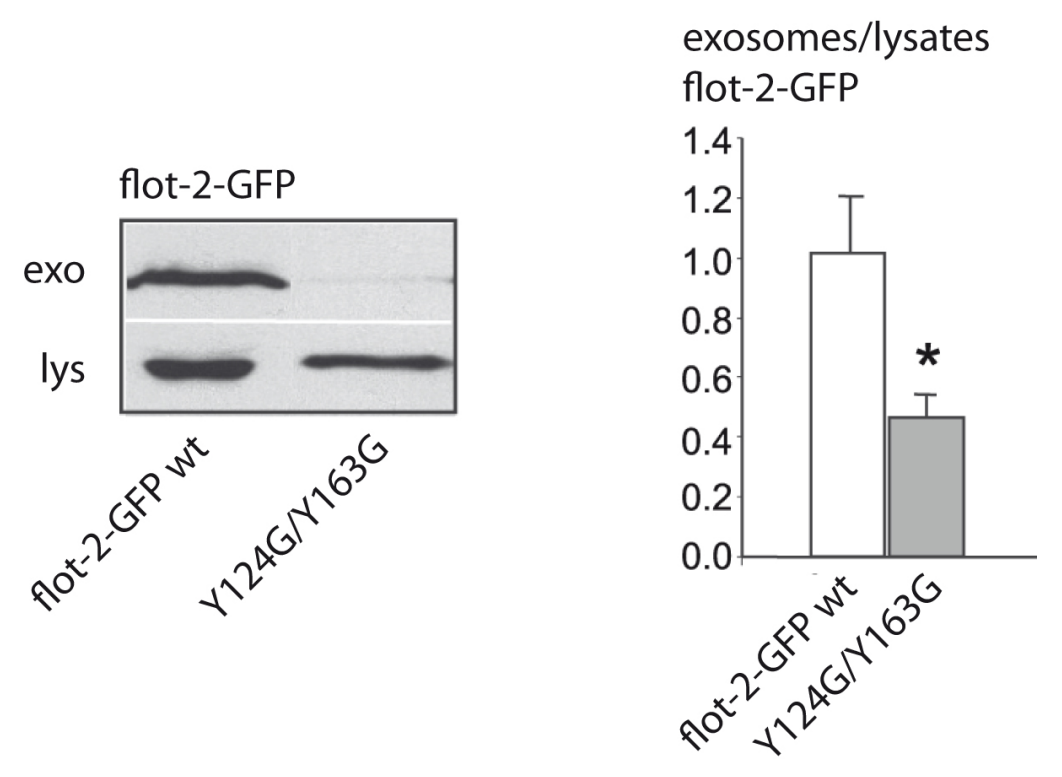

Figure 3.12: CRAC domains are necessary for the exosomal release of flotillin-2. Western blotting of exosome pellets (exo) and cell lysates (lys) from Oli-neu cells transfected with wild-type flotillin-2-GFP (flot-2-GFP wt) or the CRAC double mutant (Y124G/Y163G). Blots were scanned and intensities of the bands were quantified. The histogram shows the ratio of wild-type flotillin-2GFP (white bar) or CRAC double mutant flotillin-2-GFP (gray bar) intensities in exosome pellets versus respective cell lysates. Exosomal release of the CRAC double mutant Y124G/Y163G was impaired. Controls were normalized to 1 . Values are given as mean + SEM from $n=13$ experiments. * indicates $\mathrm{p}<0.05$.

These data indicate that the presence of both CRAC domains facilitates the translocation of flotillin-2 from the plasma membrane to endosomal compartments, and finally its incorporation into exosomes.

\subsubsection{Flotillin-2 down-regulation impairs exosomal cholesterol release}

After demonstrating that intracellular trafficking and exosomal release of flotillin-2 are regulated by cholesterol, we wondered whether flotillin-2 is required for the secretion of cholesterol via exosomes.

To approach this question, we down-regulated expression of flotillin-2 in Oli-neu cells with siRNA and measured the amount of exosome-released cholesterol. As shown previously, siRNA mediated knock-down of flotillin-2 simultaneously results in down-regulation of flotillin-1 (119). Flotillin-2 siRNA or control siRNA was introduced into cells (knock- 
down efficiency $80 \%$, see Figure 3.13B). Cells were then starved for $4 \mathrm{~h}$ in serum free SATO medium, and subsequently incubated for $4 \mathrm{~h}$ with $50 \mu \mathrm{g} / \mathrm{ml}$ cholesterol/methyl- $\beta$ cyclodextrin complex (saturated with ethanol dissolved cholesterol). We then harvested cell lysates and prepared exosomes from the culture medium. Cholesterol of exosome pellets and cell lysates was extracted with methanol/chloroform and cholesterol contents were quantified by gas chromatography. Upon flotillin-2 down-regulation the ratio of exosomal to cell lysate cholesterol was significantly decreased compared to controls $(33.38 \%$ reduction, S.E. $=13.21 \%, \mathrm{n}=8, \mathrm{p}=0.03$, Mann-Whitney $\mathrm{U}$ test) (Figure 3.13 ). This indicates that release of cholesterol via exosomes at least partially depends on flotillin-2. 
(A)

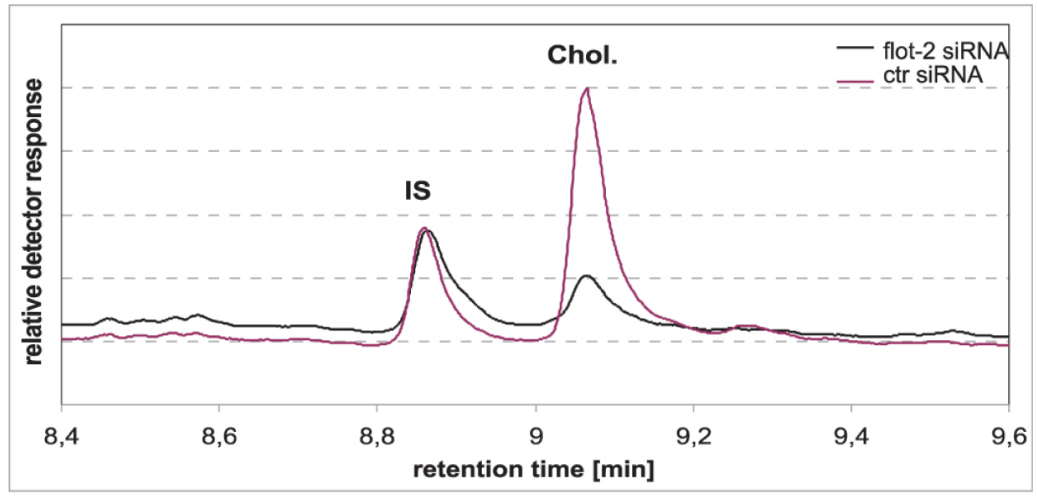

exosomes/lysates

cholesterol

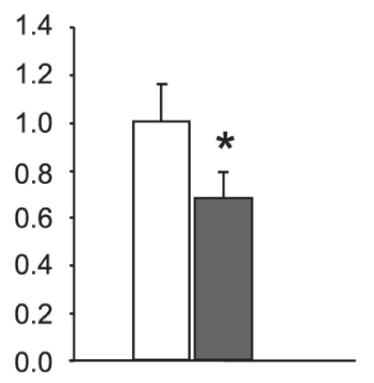

(B) ctr siRNA flot-2 siRNA flotillin-2/calnexin

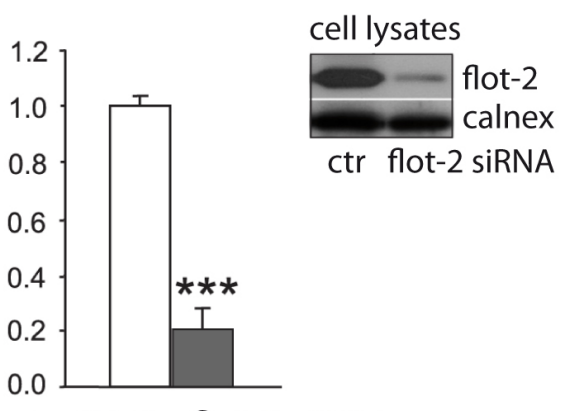

ctr siRNA flot-2 siRNA

Figure 3.13: Flotillin-2 is required for the exsosomal release of cholesterol. Oli-neu cells were transfected with siRNA directed against flotillin-2 (flot-2) or with control siRNA (ctr). Methanol/chloroform extracted organic phases from exosomal pellets and corresponding cell lysates were subjected to gas chromatography to quantify cholesterol contents. (A) A typical retention profile is shown for cholesterol extractions from exosome pellets of flotillin-2 knock-down cells (black) and control cells (red). Lanosterol (Is) served as an internal standard. (B) Ratios of cholesterol amounts from exosome pellets versus parent cell lysates were calculated for cells with siRNA-mediated down-regulation of flotillin-2 (gray bar,left histogram) and for control cells (white bar). Exosomal cholesterol release was decreased after down-regulation of flotillin-2. Efficiency of siRNA-mediated flotillin-2 down-regulation is shown in the right histogram and Western blot. Cells were lysed in CHAPS buffer and analyzed by Western blotting for signal intensities of flotillin-2 (upper lane) and calnexin (lower lane) as an internal standard. The ratio of flotillin-2 versus calnexin was calculated for cells treated with control siRNA (white bar) and flotillin-2 siRNA (gray bar). Knock-down efficiency was $80 \%$. Values of controls were normalized to 1 . Values are given as mean + SEM for $n=5$ and 9 experiments. ${ }^{*}$ indicates $p<0.05,{ }^{* * \star} p<0.0005$. 


\subsection{Exosomal cholesterol release is up-regulated in Niemann-Pick type $\mathrm{C}$ disease (NPC)}

\subsubsection{Exosome release is enhanced in NPC}

As shown in Section 3.1.2.2, cholesterol loading of cells enhances exosome secretion. Adapting exosome release to cellular cholesterol levels may be important in diseases, which are accompanied by an imbalanced lipid trafficking. One example of such a disease is the lipid storage disease Niemann-Pick type C (NPC). In NPC, vast amounts of unesterified cholesterol accumulate in late endosomal/lysosomal compartments. We therefore wanted to know whether NPC cells up-regulate their exosome secretion.

We approached the study of exosome release in NPC in several ways. In a first set of experiments we used a pharmacological model of the disease. To mimic the molecular phenotype of NPC, Oli-neu cells were treated with U18666A. This hydrophobic amine causes an impaired transport of cholesterol from late endosomes/lysosomes to the ER (259), as seen in NPC. We compared control cells and cells incubated for $16 \mathrm{~h}$ with $1.5 \mu \mathrm{g} / \mathrm{ml}$ U18666A. Exosome secretion was quantified by signals of the endogenous exosomal marker proteins, flotillin-2 and alix, and the transiently expressed EGFP-CD63. We detected a pronounced increase in exosome release for U18666A-treated cells (flotillin-2: increase to 307\%, SEM $=77 \%, \mathrm{n}=8, \mathrm{p}=0.02$, Student's t test; alix: increase to $262 \%, \mathrm{SEM}=105.1 \%, \mathrm{n}=15, \mathrm{p}$ $=0.046$, Mann-Whitney U test; EGFP-CD63: increase to $188 \%, \mathrm{SEM}=60 \%, \mathrm{n}=15, \mathrm{p}=$ 0.095, Mann-Whitney U test) (Figure 3.14A). This implies that late endosomal/lysosomal accumulation of cholesterol promotes exosome secretion.

We wanted to know whether the up-regulation of exosome release is specific for cholesterol accumulation in late endosomes/lysosomes and not in the ER. Therefore, we treated cells with a drug to accumulate cholesterol in the ER. The compound CP-113.818 is an inhibitor of acyl-coenzyme A:cholesterol acyltransferase (ACAT), an ER-resident enzyme catalyzing the esterification of cholesterol. If this reaction is blocked, unesterified cholesterol is trapped in the ER. In contrast to the treatment with U18666A, administration of $10 \mu \mathrm{M}$ CP-113.818 for $40 \mathrm{~h}$ had no effect on exosome secretion $(4.20 \%$ decrease, $\mathrm{SEM}=$ $6.11 \%, \mathrm{n}=5, \mathrm{p}=0.5$, Mann-Whitney $\mathrm{U}$ test) (Figure 3.14B).

In a second approach to mimic NPC, we down-regulated expression of NPC1 protein. The late endosomal cholesterol accumulation in NPC is caused by mutations in the npc1 or npc2 gene (200). These mutations lead to a loss of function of NPC1 or NPC2 proteins, respectively. $95 \%$ of all NPC cases are caused by mutations in npc1. To mimic NPC in 

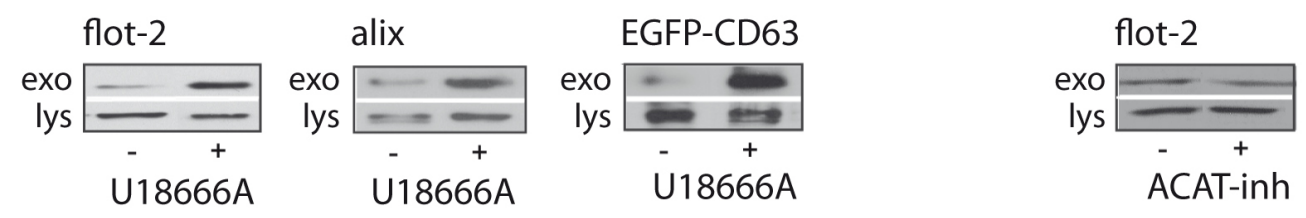

exosomes/lysates

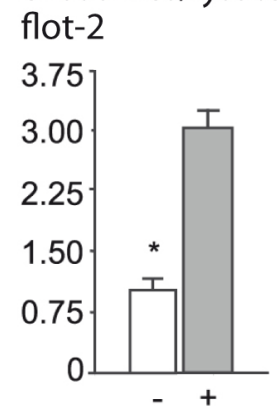

(A)

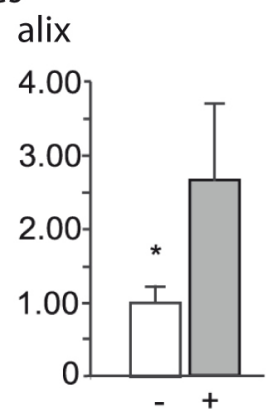

U18666A

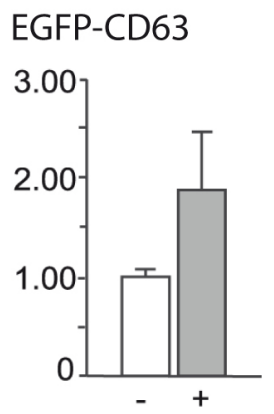

U18666A exosomes/lysates flot-2

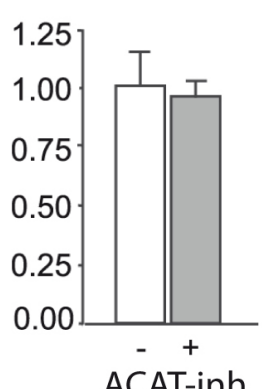

Figure 3.14: Cholesterol accumulation in late endosomes/lysosomes but not in the ER increases exosome release. (A) To accumulate free cholesterol in late endosomal/lysosomal compartments, Oli-neu cells were treated with $1.5 \mu \mathrm{g} / \mathrm{ml} \mathrm{U18666A}$ for $16 \mathrm{~h}$ (+ U18666A). Control cells were mock treated (- U18666A). Exosome pellets (exo) and corresponding cell lysates (lys) were analyzed by Western blotting (top) for endogenous exosomal marker proteins flotillin-2 (flot2) and alix, and for the transiently expressed exosomal marker EGFP-CD63. For quantification of exosome release, the ratio of protein intensity in the exosomal fraction versus protein intensity of the cell lysate was determined (bottom). For all three proteins immunoreactivity was increased in the exosome fraction after treatment with U18666A (gray bars) compared to controls (white bars). (B) To accumulate free cholesterol in the ER, cells were treated for $40 \mathrm{~h}$ with $10 \mu \mathrm{M}$ ACATinhibitor CP-113.818 (+ ACAT-inh). As controls, cells were mock treated (- ACAT-inh). Western blotting (top) of exosome pellets and parent cell lysates was conducted. For quantification of exosome release, the ratio of flotillin-2 signal intensity in exosome pellets versus cell lysates was determined (bottom). No significant difference in exosome release between treated cells and untreated controls was detected. (A+B) Values are given as mean + SEM from $n=8$ experiments with controls normalized to $1 .{ }^{*}$ indicates $p<0.05$. 
Oli-neu cells, we therefore down-regulated NPC1 protein expression by siRNA. The knockdown efficiency was approximately 60\% (Figure 3.15A). Exosome release of cells treated with siRNA directed against NPC1 was significantly increased compared to treatment with control siRNA (82.1\% increase, $\mathrm{SEM}=16.5 \%, \mathrm{n}=9, \mathrm{p}=0.001$, Mann-Whitney $\mathrm{U}$ test).

Our third model was a Chinese hamster ovary ( $\mathrm{CHO}$ ) cell line, which does not express functional NPC1. The CHO line CT43 contains a mutation in npc1 that causes expression of an NPC1 protein truncated after amino acid 933. This results in complete loss of NPC1 function (260). The CT43 clone was derived from the CHO line 25RA, which originally was selected for a gain of function mutation of SCEBP cleavage-activating protein (SCAP) (261). In the process of CT43 cell line propagation however, the mutated SCAP allele reverted back to a wild-type allele (T.Y. Chang, personal communication). For this reason, we used the wild-type $\mathrm{CHO}$ line $\mathrm{K} 1$ as a control. Exosome secretion was measured for the wild-type and the mutant CHO line by transfecting cells with flotillin-2-GFP as a marker for exosome release. Exosome pellets as well as parent cell lysates of transfected cells were subjected to Western blotting and probed against flotillin-2-GFP. Exosome release was up-regulated almost 3 times in NPC1-mutant CT43 cells compared to wild-type K1 cells (increase to $278 \%, \mathrm{SEM}=23 \%, \mathrm{n}=9, \mathrm{p}=0.0004$, Mann-Whitney U test) (Figure 3.15B). We finally wanted to verify the results, which we obtained from our pharmacological, siRNA mediated, and CHO models of NPC, in NPC patients. We obtained primary skin fibroblasts from a patient harboring the compound heterozygous NPC1 mutation R934X/P1007A and from a healthy volunteer. Fibroblasts were starved in serum free growth medium (1\% LPS) for $24 \mathrm{~h}$ proceeded by another $24 \mathrm{~h}$ of incubation in growth medium containing 10\% FCS. These growing conditions achieved a high accumulation of cholesterol in intracellular vesicles. Figure 3.16A depicts a pronounced vesicular filipin staining in fibroblasts from the NPC1 patient compared to control fibroblasts. Exosomes were then collected for $4 \mathrm{~h}$ in serum free medium after washing the cells with PBS. To determine exosome secretion, flotillin-2 signal in exosome pellets versus cell lysates was quantified by Western blotting. We have to note that general differences in flotillin-2 expression between NPC1 mutant and wild-type fibroblasts would have biased our results on exsome secretion. To rule out this possibility, we probed the Western blot membranes of cell lysates for flotillin-2 and the housekeeping protein calnexin. Flotillin-2 to calnexin ratios were similar in cell lysates from the NPC patient and the healthy control (data not shown). Exosome secretion was significantly up-regulated in fibroblasts with 


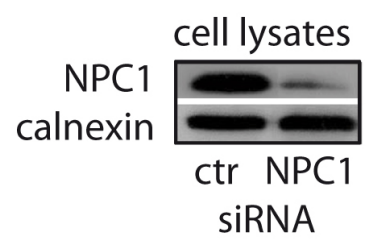

NPC1/calnexin

(A)

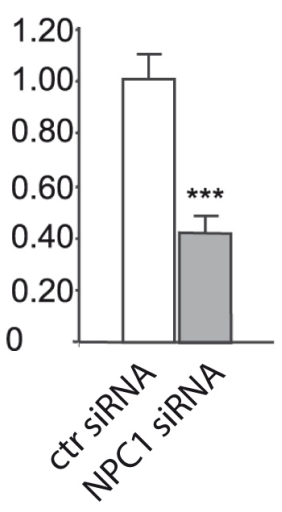

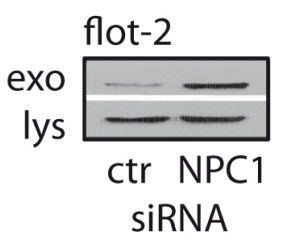

exosomes/lysates

flot-2

2.50

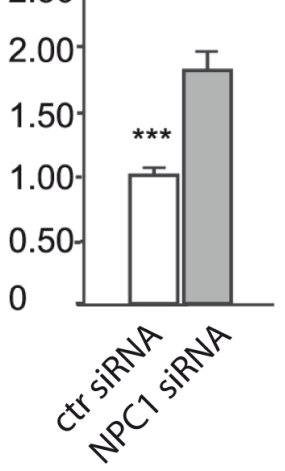

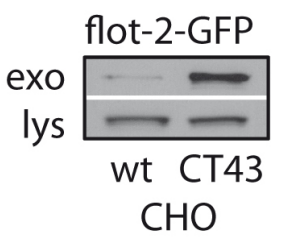

exosomes/lysates

flot-2-GFP

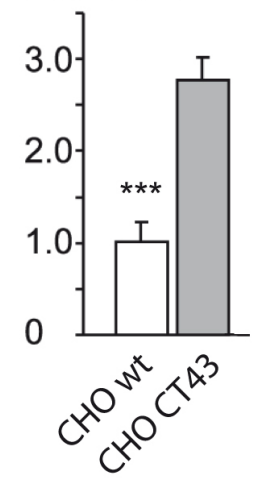

(B)

Figure 3.15: Loss of functional NPC1 increases exosome release. (A) Oli-neu cells were transfected with siRNA directed against NPC1 or with control siRNA (ctr). To test knock-down efficiency, cells from NPC1 siRNA and control siRNA treatment groups were scraped into CHAPS lysate buffer and subjected to Western blot (top, left). The ratio of signal intensities for NPC1 versus calnexin (as loading control) was determined (bottom, left). Efficiency of NPC1 downregulation was $58 \%$. For determination of exosome release, Western blotting (top, middle) of exosome pellets (exo) and corresponding cell lysates (lys) of both treatment groups was conducted and the ratio of flotillin-2 signal intensity in exosome pellets versus cell lysates was calculated (bottom, middle). Upon NPC1 knock-down, exosome release was increased. (B) The wild-type Chinese hamster ovary ( $\mathrm{CHO}$ ) cell line $\mathrm{K} 1$ (wt) and the $\mathrm{CHO}$ cell line CT43 were analyzed for exosome secretion. CT43 cells habour a truncation mutation in the npc1 gene leading to loss of function of the NPC1 protein. Western blots (top, right) of exosome pellets and corresponding cell lysates were stained for transiently expressed flotillin-2-GFP (flot-2-GFP). The ratios of signal intensities for exosome pellets versus cell lysates showed that CT43 cells have an increased exosome release (bottom, right, gray bar) compared to wild-type $\mathrm{CHO}$ cells (white bar). ( $\mathbf{A}+\mathbf{B}$ ) Values are given as mean + SEM from $n=9$ experiments with controls/wild-type normalized to 1 . *** indicates $\mathrm{p}<0.0005$. 
the R934X/P1007A mutation in NPC1 (increase to 477.15\%, $\mathrm{SEM}=236.56 \%, \mathrm{n}=10$, $\mathrm{p}=0.0044$, Mann-Whitney U test) (Figure 3.16B).

Taken together, these data indicate that loss of NPC protein function results in an increased exosome secretion. Convincingly, the same result could be replicated in four different model systems, including pharmacological manipulation, protein down-regulation, mutation of the npc1 gene in CHO cells, and analysis of NPC patient fibroblasts. 


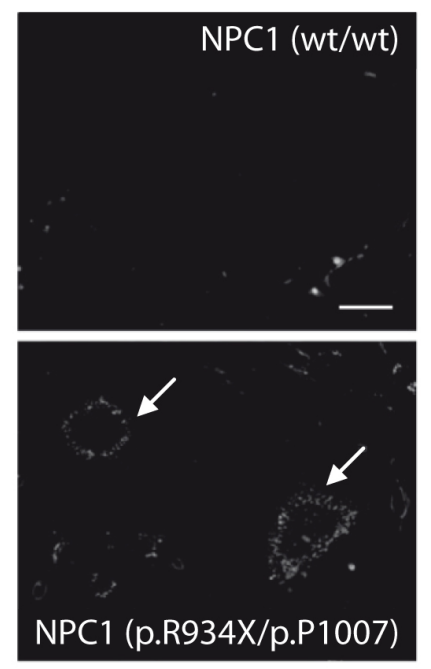

(A)

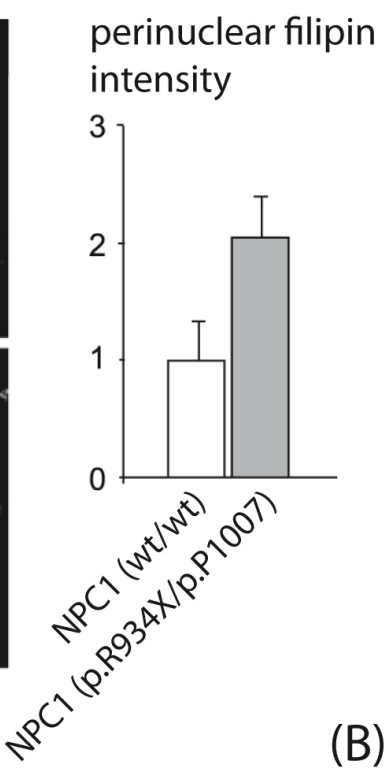

(B) exosomes/lysates

flot-2

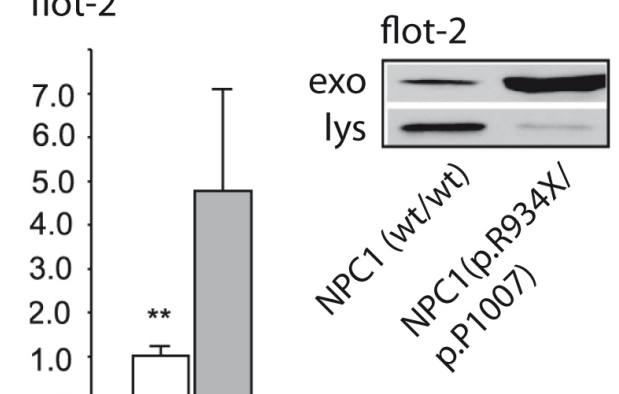

Figure 3.16: Exosome release is increased in human skin fibroblasts derived from an NPC patient. Human skin fibroblasts derived from a patient bearing the compound heterozygous mutations p.R934X/p.P1007A and from a healthy control patient were cultured in vitro. (A) Fibroblasts were fixed and stained with filipin for free cholesterol. Fluorescence images were acquired automatically using a wide-field microscope with a 10× objective. Cholesterol accumulation could be detected for fibroblasts with mutant NPC1 (bottom, left). Scale bar: $10 \mu \mathrm{m}$. Perinuclear filipin signal intensities (arbitrary units) were measured from 200 individual cells per cell line and quantified as described by Bartz et al. 2009 (262) with modifications for cultured fibroblasts (H. Runz, unpublished data) (left histogram). (B) To determine exosome secretion from cultured fibroblasts, exosome pellets and corresponding cell lysates were subjected to Western blotting (right) and probed with an antibody against endogenous flotillin-2 (flot-2). The ratios of flotillin-2 signal intensities in exosomal fractions versus cell lysates were calculated. Fibroblasts from the NPC1 patient showed increased exosome release (gray bar, right histogram) compared to fibroblasts from the healthy control (white bar). Values are given as mean + SEM from $n=10$ experiments. ** indicates $p<0.005$. 


\subsubsection{Cholesterol release via exosomes is increased in NPC}

In summary, we found that intracellular cholesterol accumulation promotes exosome secretion. We therefore asked whether exosomes could contribute to cholesterol release in response to rising cellular cholesterol levels. We quantified the amount of exosome-released cholesterol for two of our models: Oli-neu cells treated with U18666A and CHO CT43 cells expressing non-functional NPC1. Cholesterol was extracted from exosome preparations and corresponding cell lysates and was subsequently measured by gas chromatography. When Oli-neu cells were treated with $1.5 \mu \mathrm{g} / \mathrm{ml}$ U18666A, cholesterol secretion within exosomes was indeed increased ( $78 \%$ increase, $\mathrm{SEM}=13.4 \%, \mathrm{n}=9, \mathrm{p}=0.0049$, Mann-Whitney U test) (Figure 3.17A). A similar up-regulation of exosomal cholesterol release was also seen for the $\mathrm{CHO}$ cell line CT43 (increase to $174 \%, \mathrm{SEM}=15 \%, \mathrm{n}=6$, $\mathrm{p}=0.002165$, Mann-Whitney U test) (Figure 3.17B).

We therefore conclude that exosomal cholesterol release is up-regulated under conditions of increased cellular cholesterol, and might partially bypass the late endosomal/lysosomal accumulation of free cholesterol in NPC disease.

\subsubsection{Exosome release can be reduced by transfection of wild-type NPC1 in NPC1-mutants}

Since the deficiency in functional NPC1 causes up-regulation in exosome secretion, expression of wild-type NCP1 in mutant cells should reverse this process. We wanted to test this assumption with the CHO cell line CT43, which expresses a truncated, non-functional NPC1 protein. The CHO line K1 served as a control since it produces wild-type NPC1. CHO cells of the K1 line (wild-type) and the CT43 line (mutant) were transfected with flotillin-2-GFP as a marker for exosomes. To quantify exosome secretion, exosome pellets and cell lysates were analyzed for flotillin-2-GFP signal with Western blotting. Basic levels of exosome release of the mutant CT43 and wild-type K1 CHO line were determined. In Figure 3.15B, we show that CHO CT43 cells secrete more exosomes compared to K1 cells. We now asked whether transfection with wild-type NPC1 can restore normal, low levels of exosome release in CT43 cells. To approach this question, we co-transfected CT43 and K1 cells with flotillin-2-GFP and either mutant or wild-type EYFP-NPC1, and measured exosome secretion of the cells. If our hypothesis holds true, wild-type EYFP-NPC1 should reduce exosome secretion in CT43 cells. Transfection with mutant EYFP-NPC1 P692S, which has a reduced activity (213), should have a smaller impact. 
exosomes /lysates
cholesterol

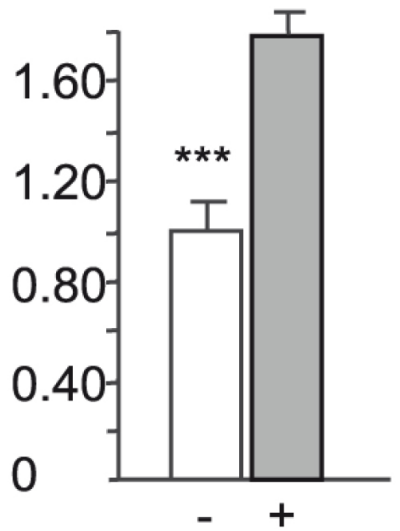

(A)
U18666A exosomes /lysates

cholesterol

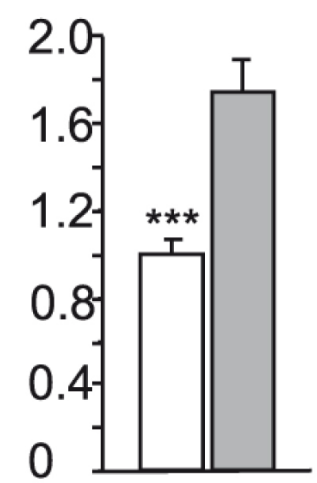

(B)

Figure 3.17: Exosomal cholesterol release is increased in NPC. (A) Oli-neu cells were treated with $1.5 \mu \mathrm{g} / \mathrm{ml} \mathrm{U} 18666 \mathrm{~A}$ for $16 \mathrm{~h}(+\mathrm{U} 18666 \mathrm{~A})$ to accumulate free cholesterol in late endosomes/lysosomes. Controls were mock treated (-U18666A). (B) The Chinese hamster ovary (CHO) wild-type cell line $\mathrm{K} 1$ (wt), and a $\mathrm{CHO}$ mutant cell line lacking functional NPC1 (CT43) were compared for cholesterol release via exosomes. (A+B) After methanol/chloroform extraction of organic phases from exosomal pellets and corresponding cell lysates, samples were subjected to gas chromatography to quantify cholesterol contents. Ratios of cholesterol amounts from exosome pellets versus parent cell lysates were calculated for control cells (white bars) and U18666A treated cells or NPC1 mutants, respectively (gray bars). Exosomal cholesterol release was increased in cells with cholesterol accumulation in late endosomes/lysosomes in both experiments. Values of controls were normalized to 1 . Values are given as mean + SEM for $n=9$ experiments. ${ }^{* * *}$ indicates $p<0.0005$. 
We first wanted to exclude that our results are biased by different expression rates of the two EYFP-NPC1 constructs. Therefore, lysates of equal amounts of transfected CHO K1 cells were probed against GFP/EYFP in Western blotting. We could show that expression rates of transfected wild-type EYFP-NPC1 and mutant EYFP-NPC1 P692S were similar, as were the expression rates of flotillin-2-GFP (Figure 3.18).

Co-transfection of mutant or wild-type EYFP-NPC1 did not affect the release of flotillin2-GFP positive exosomes in wild-type K1 CHO cells (Figure 3.19A). In contrast, exosome secretion was dramatically reduced by EYFP-NPC1 co-transfection in cells of the mutant CT43 line (Figure 3.19B).

We then quantified these data (Figure 3.19C). Co-transfection of CT43 cells with wild-type EYFP-NPC1 reduced exosomal flotillin-2-GFP release to the basic level of K1 wild-type cells $(107 \%$ as compared with wild-type $\mathrm{CHO}$ with $100 \%, \mathrm{SEM}=24 \%, \mathrm{n}=14, \mathrm{p}=1$, Mann-Whitney $U$ test). In contrast, exosome release was only partially reduced when CT43 cells were co-transfected with EYFP-NPC1 bearing the point mutation P692S (28\% reduction as compared with flotillin-2-GFP transfected CT43 cells, SEM $=45 \%, \mathrm{n}=14$, $\mathrm{p}=0.041$, Mann-Whitney $\mathrm{U}$ test). Most probably, the smaller effect of EYFP-NPC1 P692S mutant on exosome secretion is caused by the reduced function of the protein (213).

Our results demonstrate that the introduction of wild-type NPC1 in NPC1 mutant cells reduces an up-regulated exosome secretion to base level. With this experiment we prove that the release of exosomes is adjusted to cellular cholesterol accumulation caused by loss of functional NPC1. Expression of functional NPC1 is sufficient to restore normal exosome secretion.

In summary, this last section highlighted the role of exosomes in the cholesterol storage disease NPC, where exosomes contribute to the release of accumulated cholesterol from cells. 
CHO wt lysates:

anti-GFP

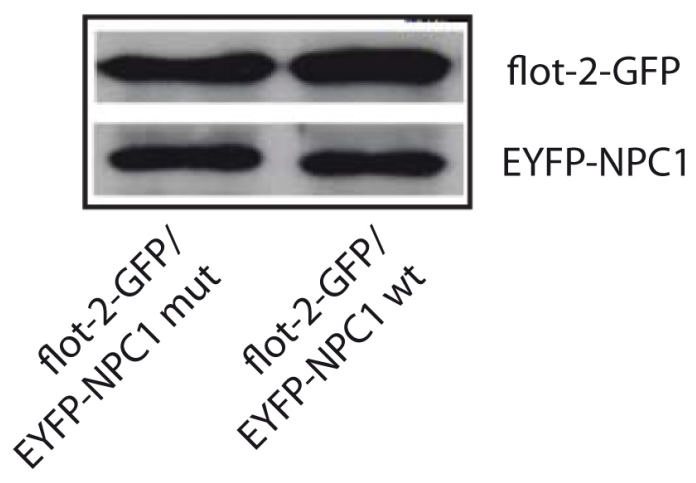

Figure 3.18: Transfection rates of wild-type and mutant NPC1 are similar. The wild-type (wt) Chinese hamster ovary $(\mathrm{CHO})$ cell line $\mathrm{K} 1$ was co-transfected with flotillin-2-GFP and either mutant EYFP-NPC1 P692S (mut) or wild-type EYFP-NPC1 (wt). Cell lysates were scratched in CHAPS buffer and subjected to Western blotting. Probing with GFP antibody detected similar expression levels of flotillin-2-GFP, wild-type EYFP-NPC1, and mutant EYFP-NPC1 P692S. 
CHO wt:

flot-2-GFP
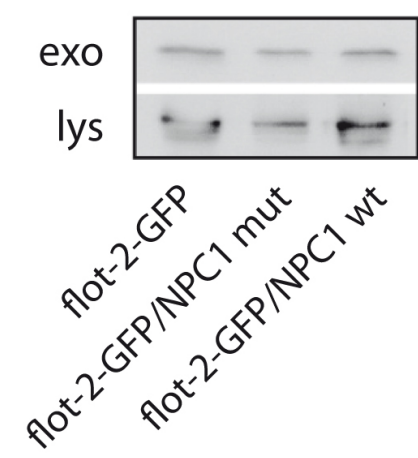

(A)
CHO CT43:

flot-2-GFP
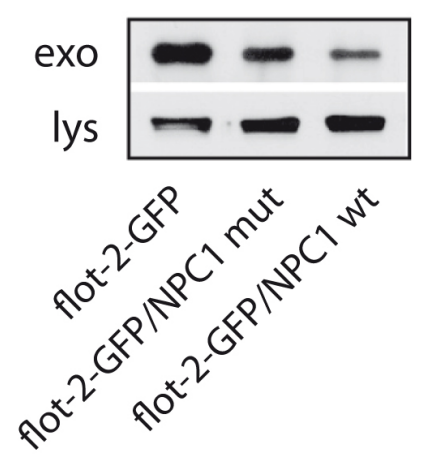

exosomes /lysates

flot-2-GFP

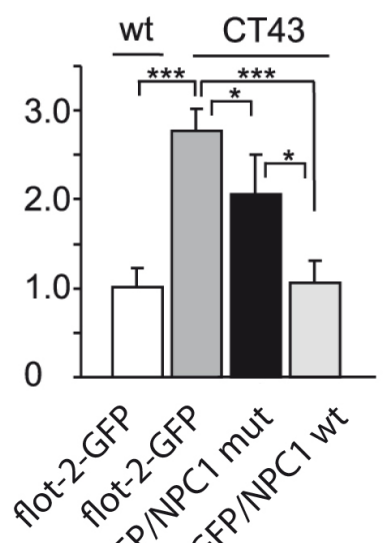

(C)

(B)

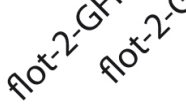

Figure 3.19: Transfection of NPC1-mutant CHO cells with wild-type NPC1 restores normal exosome release. (A) Cells of the wild-type Chinese hamster ovary cell line $\mathrm{K} 1$ ( $\mathrm{CHO}$ wt) were transfected with either flotillin-2-GFP alone (flot-2-GFP, left) or co-transfected with a combination of flotillin-2-GFP and mutant EYFP-NPC1 P692S (NPC1 mut, middle) or wild-type EYFP-NPC1 (NPC1 wt, right). Exosome fractions and lysates of corresponding parent cells were subjected to Western blotting and probed against flotillin-2-GFP with GFP antibody. (B) Same experiment as in (A) with the $\mathrm{CHO}$ cell line $\mathrm{CT} 43$, which lacks functional NPC1. (C) The histogram shows the ratios of flotillin-2-GFP signal intensities of exosome pellets versus cell lysates. Transfection of mutant or wild-type EYFP-NPC1 can partially (black bar) or completely reduce (light gray bar) exosome release to wild-type levels (white bar). Values are given as mean + SEM for $n=9$ experiments. Flotillin-2-GFP transfected CHO wt was normalized to $1 .{ }^{*}$ indicates $p<0.05,{ }^{* * *} p<0.0005$. 


\section{Chapter 4}

\section{Discussion}

This study aimed at elucidating the role of exosome secretion in maintenance of cellular cholesterol homeostasis. In summary, we could show that free cholesterol can be released from the cell via exosomes and that this pathway crucially depends on flotillin-2. Proving the potential of cells to adjust their exosome secretion to cholesterol levels, release of exosomes was decreased upon cholesterol depletion and increased when free cholesterol was loaded to the cells. We provide evidence for the functionality of flotillin's cholesterol interacting CRAC domains, which are important for directing the protein to intracellular vesicles and eventually to exosomes. We further propose that flotillin-2 fulfills a role in directing cholesterol into the exosome pathway, since down-regulation of flotillin-2 impaired exosomal cholesterol release. Under the pathological condition of Niemann-Pick type C disease, where vast amounts of free cholesterol accumulate in late endosomes and lysosomes, cholesterol release via exosomes was found to be up-regulated. This may serve as a mechanism to partially bypass the cholesterol traffic block. Taking these data together, we propose a novel role for exosomes and flotillin-2 in shuttling cholesterol from the cell and thereby contributing to the regulation of cholesterol homeostasis.

\subsection{Flotillin-2 is distributed in response to cholesterol levels}

\subsubsection{Flotillin-2 distribution depends on cholesterol}

Flotillin-2, a protein abundantly found in MVBs and exosomes, has previously been suggested to associate with lipid-enriched membrane microdomains via interaction with cholesterol (120). This led us to investigate its localization and trafficking in dependence of cellular cholesterol levels. 
In a first step, co-localization of flotillin-2 with different endosomal markers was tracked by immunofluorescence microscopy in Oli-neu cells. We found that flotillin-2 localizes partially to recycling endosomes, and in larger amounts to late endosomes and lysosomes. Only a minor fraction of the protein was located at the plasma membrane. Flotillin-2 was previously demonstrated to localize mainly to the plasma membrane $(104 ; 254 ; 255)$ where it forms homo-oligomers and hetero-oligomers together with flotillin-1 (119). There are however studies that found flotillins abundantly in vesicular compartments, such as recycling endosomes, lysosomes and MVBs $(107 ; 108 ; 37 ; 256)$. Together with our findings, these data suggest that subcellular flotillin-2 distribution depends on cell type. Interestingly, it was shown that flotillin localization to lysosomal structures and the plasma membrane appears to be clustered, and that flotillins co-localize with $\mathrm{BC} \theta$-staining of free cholesterol (111). Cholesterol has been assumed to be crucial for flotillin-2 assembly at detergent resistant membranes before (263). Accordingly, Domingues and colleagues reported flotillin-2 to be found almost exclusively in Triton X-100 isolated detergent resistant membranes under natural conditions. Methyl- $\beta$-cyclodextrin induced cholesterol depletion could however partially redistribute the protein to detergent-soluble membrane fractions (264).

Having determined the intracellular distribution of flotillin-2 in Oli-neu cells, we further asked whether the protein's localization is sensitive to changes in cellular cholesterol levels as indicated by the previously discussed studies. Therefore, we incubated cells with cholesterol modulating drugs. Acute or long-term cholesterol depletion by administration of methyl- $\beta$-cyclodextrin or simvastatin, respectively, induced a notable redistribution of flotillin-2 to the plasma membrane. Conversely, application of cholesterol to Oli-neu cells led to an accumulation of large amounts of flotillin-2 in intracellular vesicular structures. Flotillin-2 co-localized there with filipin-stained free cholesterol. The same pronounced intracellular accumulation of flotillin-2 and its removal from the plasma membrane was observed when we pharmaceutically arrested cholesterol in late endosomes/lysosomes by U18666A, or in the ER by inhibition of its esterification via ACAT. In accordance with these findings, cholesterol-dependence of cellular flotillin-2 cycling was previously demonstrated for HeLa cells by Langhorst and colleagues. While methyl- $\beta$-cyclodextrin-mediated cholesterol depletion resulted in a $40 \%$ reduction of flotillin-2 trafficking density (mobile molecules per total cell area), supplying the cell with LDL-derived cholesterol could significantly increase the mobility of flotillin-2 (256). 


\subsubsection{Flotillin's CRAC domains facilitate its interaction with cholesterol}

Next, we were interested in how the effect of cholesterol on cellular flotillin-2 distribution is mediated at a molecular level. The cholesterol recognition/interaction amino acid consensus (CRAC) sequence is a homologous domain shared by various proteins, which are associated to cholesterol. This domain was first identified in the peripheral type benzodiazepine receptor (PBR) by $\mathrm{Li}$ and colleagues. The authors showed that CRAC mediates the translocation of cholesterol into mitochondria $(121 ; 122)$. When PBR's CRAC motif was mutated at a tyrosine residue, uptake of cholesterol and its mitochondrial transport were impaired (123). CRAC domains were further detected in several other proteins, including caveolin, a protein involved in the maintenance of cellular cholesterol homeostasis, where it promotes membrane recruitment of cholesterol $(121 ; 124)$.

The protein sequence of flotillin-2 contains two sequences fulfilling the required consensus for CRAC domains (120). These sequences might possibly be responsible for the accumulation of cholesterol at membrane microdomains. However, the functionality of these domains in cholesterol binding has not been validated so far for flotillin-2. To assess their importance for cholesterol-dependent flotillin-2 trafficking, we mutated flotillin-2 at the tyrosine residues of one or both putative CRAC domains at positions Y124G and Y163G. The resulting CRAC mutants were compared to wild-type flotillin-2 for their subcellular distribution and secretion via exosomes. As predicted, the single and double mutation of CRAC domains reduced flotillin-2 localization to vesicular endosomal compartments and retained most of the mutated protein residing at the plasma membrane. The CRAC double mutant showed the strongest plasma membrane retention phenotype, and it additionally was largely excluded from exosomes. These findings support the idea that cholesterol-dependent sorting of flotillin-2 is mediated on a structural level through its CRAC domains. Flotillin-2 might directly bind to cholesterol, and thereby be targeted to membrane microdomains highly enriched in this lipid. However, we have to note that our experiments did not directly demonstrate the cholesterol binding of CRAC domains and that further investigations are needed to prove a direct interaction.

Flotillin-2, which would bind to cholesterol at the plasma membrane, could be endocytosed together with cholesterol and directed to MVBs. The loss of functional CRAC domains could possibly impair this process leading to the observed phenotype. Supporting this hypothesis, a recent study by Neumann and colleagues showed that EGF-mediated endocytosis of flotillin-2 is inhibited by mutating the second CRAC domain at the Y163 residue (126). EGF-signaling leads to an activation of the Src kinase, which subsequently phos- 
phorylates flotillin-2 at multiple tyrosine residues. Hence, the authors speculated that the reduced incorporation of flotillin-2 into endosomes is due to loss of the phosphorylation site Y163 (126). However, we did not detect any difference in the cellular distribution of endogenous flotillin-2 and transiently expressed flotillin-2-GFP in Oli-neu cells upon treatment with PP-2, an inhibitor of Src kinase (unpublished data by Sievert Weiss). We therefore propose that the interaction of flotillin- 2 with cholesterol via the CRAC domains - rather than flotillin-2 phosphorylation at Y163 - is triggering the protein's endocytosis.

\subsection{Flotillin-2 recruits cholesterol to exosomes}

\subsubsection{Exosomal release of cholesterol depends on flotillin-2}

We demonstrate in this study that the subcellular distribution of flotillin-2 to endosomal membranes is regulated by cholesterol. Furthermore, flotillins were previously shown to associate with cholesterol-rich membrane microdomains (111), and facilitate a cholesteroldependent endocytosis pathway (265). Since cholesterol and flotillin-2 also co-localize within endosomal compartments (see results Section 3.1.1.3), we proposed that cholesterol trafficking to MVBs (and thus eventually to exosomes) depends on flotillin-2.

Our results show that exosomal cholesterol release decreases after siRNA mediated downregulation of flotillin-2. As previously demonstrated, flotillin-2 stabilizes flotillin-1 by forming hetero-tetramers. Hence, down-regulation of flotillin-2 induces degradation of flotillin-1 via the proteasome (119). On the other hand, down-regulation of flotillin-1 does not affect flotillin-2 protein levels (119). We therefore conclude from our experiments that flotillin-2 participates in directing cholesterol to exosomes. Further experiments with a selective down-regulation of flotillin-1 will allow to make assumptions about the role of flotillin-1 in this process. Flotillin-2-dependent distribution of cholesterol was also shown by Roitbak and colleagues. Yet in some transfected cells overexpressing flotillin2 the authors observed cholesterol redistribution to the plasma membrane (120). We could, however, show that flotillin-2, which is mutated at its cholesterol-interaction sites (CRAC), remains at the plasma membrane, while wild-type flotillin-2 preferentially locates to vesicular endosomal structures.

Sorting of cholesterol to exosomes therefore seems to be mediated by flotillins at the level of endocytosis and MVB formation. As a consequence of this pathway, MVBs may be especially enriched in cholesterol. Indeed, immuno-electron microscopic analysis of endocytic compartments in human B lymphocytes revealed that $45 \%$ of the cell's total 
endocytic cholesterol was located to vesicles with the typical morphology of MVBs (266). The intraluminal vesicles of MVBs harbored most of this cholesterol, i.e. $39 \%$, while only $6 \%$ were found at the MBV-limiting membrane (266). This is in accordance with the detected cholesterol enrichment of exosomes derived from B lymphocytes (9).

\subsubsection{Flotillin-dependent endocytosis}

Confocal images demonstrating flotillins' punctuate distribution at the plasma membrane suggest their assembly at lipid microdomains (133). These membrane domains, often referred to as 'lipid rafts', are liquid-ordered platforms enriched in cholesterol and sphingolipids (112). Flotillins were shown to co-localize with BC $\theta$, a marker for free cholesterol, at the plasma membrane (111). Similarly to caveolins, flotillins were further detected within detergent resistant membrane fractions (98). However, their association to detergent resistant membranes as well as their targeting to lipid droplets occurs independently of caveolins (267).

Formation of caveolae and uptake of material through clathrin-coated pits are the two best-characterized routes for endocytosis in mammalian cells. Both pathways involve the presence of dynamin. Caveolin-mediated endocytosis largely depends on the association with lipid microdomains. To its cargoes belong albumin, the simian virus 40, and cholera toxin (268). The clathrin-dependent endocytic pathway facilitates the uptake of material such as LDL particles, transferrin receptors and growth factors (268). Interestingly, flotillins can also localize to structures at the plasma membrane that are distinct from clathrin coated pits and caveolae, as observed in immunofluorescence microscopy (133; 134).

Taking these observations into account, a novel clathrin- and caveolin-independent endocytic pathway has been described that is regulated by the action of flotillins $(133 ; 126)$. This pathway might be involved in the trafficking of CD59, a GPI anchored protein, and cholera toxin B, as these cargo were shown to co-localize with flotillin-1 in endosomes (133). Moreover, when flotillin-1 expression was down-regulated by siRNA, endocytosis of CD59 was reduced and cholera toxin B uptake was shifted to a dynamin-dependent route (133). To study the mechanism of flotillin-meditated endocytosis, Frick and colleagues co-overexpressed flotillin-1 and flotillin-2 in HeLa cells and monitored their localization and dynamics by confocal and electron microscopy (134). Upon co-overexpression, lateral diffusion of flotillins along the plasma membrane was reduced and an increased number of punctuated plasma membrane microdomains was formed, which contained the two pro- 
teins in a 1:1 ratio. Flotillins frequently decorated plasma membrane invaginations with a similar flak-shape as caveolae. The authors therefore proposed that flotillin-1 and flotillin2 promote internalization of membrane microdomains in a dose-dependent manner (134). However, in another study on caveolin-1 lacking fibroblasts, induction of flask-shaped plasma membrane invaginations by overexpressing flotillin- 1 and flotillin- 2 could not be reproduced (269). This indicates that other molecules additional to flotillins might be necessary to promote endocytosis.

Hence, the exact mechanism of flotillin action during endocytosis is still elusive. Other factors possibly contributing to the internalization process remain to be identified. In a recent study, tyrosine-phosphorylation of flotillin by the enzyme Fyn has been suggested as an initiating event in flotillin endocytosis (270). A role for cholesterol in the pathway remains as yet undefined. Very recently, Carcea and colleagues described a flotillinand raft-dependent endocytosis of Semaphorin 3A (Sema3A) in cortical neurons. Sema3A is a guidance cue influencing the generation of axonal trajectories (271). A subtype of cortical neurons strongly responded to Sema3A stimulation and internalized the protein via a 'lipid-raft'-mediated endocytosis pathway. Flotillin-1 was involved in this process, as down-regulation of the protein significantly decreased Sema3A internalization. The authors were interested in the molecular mechanism mediating this process. They could shown that Sema3A increases the formation of flotillin-1 clusters in growth cones. Interestingly, treatment of neurons with the cholesterol-binding agent filipin, which was used in this study as an inhibitor of raft-mediated endocytosis, impaired the cluster formation of flotillin-1. It is therefore likely that flotillin-1 is recruited to cholesterol-rich membrane microdomains in response to Sema3A and facilitates Sema3A's raft-mediated, but dynamin-independent endocytosis (271).

The possibility of cholesterol involvement in flotillin-dependent endocytosis is further supported by the observation that flotillin-1 and flotillin- 2 vesicle trafficking is about $40 \%$ reduced after cholesterol depletion via administration of methyl- $\beta$-cyclo-dextrin (256). Along the line, flotillin-mediated vesicle trafficking might contribute to the mobility of cholesterol within the cell.

\subsubsection{Parallels of flotillin- and caveolin-mediated cholesterol mobility}

The role of flotillins in regulating cholesterol mobility and homeostasis may be analogous to the function of caveolins. These major components of endocytic caveolae were suggested to bind and sequester cholesterol at the membrane (116). They are thought to 
participate in lateral organization of lipids at the plasma membrane by forming more stable microdomains that are enriched in subpopulations of lipids and depleted from others. Thereby they could modulate the potential of distinct raft domains to be endocytosed (116). Such a protein-driven plasma membrane organization of cholesterol might also be facilitated by flotillins.

Flotillins and caveolins share some structural and functional properties that may be important for this function. Both proteins form homo- and hetero-oligomers that stabilize them at the membrane $(119 ; 272 ; 273)$. Moreover, caveolin contains a membrane domain with hairpin-like topology, which integrates into the cytosolic layer of the plasma membrane. Its membrane-association is further facilitated by several palmitoylation sites. In a similar way, flotillin-2 is proposed to be anchored deeper into the membrane by its SPFH domain and N-terminal palmitoylations and myristoylations (see Figure 1.2) (116).

The CRAC domain, a sequence shared by various cholesterol interacting proteins, has been found in caveolin and flotillin-2 $(121 ; 120)$. Accordingly, caveolin was shown to directly bind to cholesterol in a 1:1 ratio (274). Studies on peptides, which contain caveolin's CRAC domain, demonstrated that the motif promotes segregation of cholesterol into cholesterolenriched domains and cholesterol-depleted membrane regions (124). Flotillin's CRAC domains might similarly facilitate lipid phase separation and flotillin targeting to evolving endosomes in conjunction with cholesterol. Our experiments with CRAC domain mutants of flotillin-2 demonstrate that this motif is involved in directing the protein to intracellular vesicular structures and exosomes. Thus, we were able to confirm a functional relevance of CRAC for flotillin-2. However, whether flotillins interact with cholesterol in a direct or indirect manner remains to be elucidated.

We could show that the translocation of flotillin-2 between the plasma membrane and intracellular vesicles depends on cellular cholesterol levels. While cholesterol loading redistributed flotillin-2 to endocytic compartments, depletion of cholesterol led to retention of the protein at the plasma membrane. Similarly, caveolin-1 distribution is highly sensitive to perturbation of the cholesterol homeostasis, as reported by Smart and colleagues (275). Expression of caveolin in response to cellular cholesterol levels is regulated at the level of transcription by binding of cholesterol to sterol regulatory elements (276).

Caveolin was demonstrated to be involved in free cholesterol efflux from the plasma membrane to HDL acceptors (277). Its overexpression enhances localization of cholesterol at caveolae and cholesterol egress (278). Caveolin was further associated with the trafficking of LDL-derived cholesterol via intracellular compartments back to the plasma membrane 
(279) and the translocation of newly synthesized cholesterol from the ER to the plasma membrane (176). In accordance with caveolin's role in cholesterol trafficking and homeostasis, cholesterol loading of cells up-regulates caveolin-1 expression (280) and caveolaedependent endocytosis of various cargo (281). Interestingly, in a murine model for NPC disease, caveolin-1 expression was also found to be significantly increased (282). However, it is still unraveled whether this phenotype actually facilitates or counteracts the disease. Flotillin-2 seems to promote cholesterol efflux from the cell by mediating its secretion via exosomes. In agreement with this assumption, we could demonstrate a decrease in exosomal cholesterol release upon siRNA mediated down-regulation of flotillin-2. This exosomal cholesterol efflux is influenced by cellular cholesterol levels, since exosome secretion was found to be up-regulated after cholesterol loading, and down-regulated upon depletion of cholesterol. Thus, analogous to caveolin, flotillin-2 seems to be modulated in its activity by cholesterol. Whether this is also facilitated at the level of gene expression remains to be clarified.

\subsection{Exosomes may contribute to cellular cholesterol homeostasis}

\subsubsection{Exosome secretion is adjusted to cholesterol levels}

We found that cells reduce the release of flotillin-2-containing exosomes in response to diminishing cholesterol pools caused by simvastatin. On the other hand, cells increase exosomal flotillin-2 and cholesterol secretion when intracellular cholesterol levels are enhanced by loading of free cholesterol or treatment with U18666A. These findings underline the involvement of exosomes in cellular cholesterol efflux. However, from these data alone we can not draw conclusions whether the absolute number of secreted exosomes is upregulated, or whether only flotillin-2- and cholesterol-enriched membrane compartments are concentrated in a single exosome particle. Both mechanisms would eventually result in the observed efflux of superfluous cholesterol from cells. To discriminate between the two possibilities, we did not only determine exosomal flotillin-2 release, but also stained for three other proteins that are enriched in exosomes, i.e. alix, CD63, and PLP. These proteins most probably partition into independent subdomains within exosomes. Alix is a member of the ESCRT machinery, which is involved in exosomal sorting of various proteins (45). Some cargo are however included into exosomes independent of ESCRT. These may rather depend on the action of tetraspanins, such as CD63, which is proposed to organize large protein complexes (35). In contrast, exosome-sorting of the proteolipid protein PLP 
was shown to require ceramide (57), and flotillin-2 is probably incorporated into exosomes through its association with 'lipid rafts' (111). Since alix, CD63, PLP, and flotillin-2 are incorporated into exosomes by four discrete processes, they are unlikely to localize to the same exosomal microdomains. However, all of the four proteins were notably increased in the exosomal fraction upon cellular cholesterol accumulation. This finding favors the hypothesis that cholesterol increases the absolute number of secreted exosomes.

An exosomal enrichment in certain lipids was shown by various studies. Depending on the cell type, these may be sphingomyelins, phosphatidylserines or gangliosides (40; 283; 9). Band T-cells were further found to secrete exosomes with increased cholesterol : phospholipid ratios $(9 ; 283)$. In contrast, exosomes of other cell types, such as erythrocytes or mast cells, did not display cholesterol enrichment $(284 ; 40)$. In a previous study of our group, the molecular composition of Oli-neu cell-derived exosomes was analyzed by nano-electrospray ionization tandem mass spectrometry. Lipid composition of exosomes strikingly resembled that of 'lipid rafts' (57). This included an almost 2.5-fold enrichment in the percentage of cholesterol compared to cellular membranes (57). It seems therefore plausible that in our experiments exosomal cholesterol efflux was facilitated by secretion of an increased number of exosomes, which were additionally enriched in cholesterol.

The cholesterol-enrichment of intraluminal vesicles/exosomes itself might even participate in the regulation of MVB-fusion with the plasma membrane (62). Membrane accumulation of cholesterol was demonstrated to facilitate recruitment of the small GTPase Rab7 to endosomes. It additionally inhibited the dynamic properties of late endocytic compartments, which became essentially immobile (285). The authors propose that Rab7 may increase minus-directed motility of endosomes, arresting them in the perinuclear region (285). Cholesterol-facilitated sorting of Rab7 to intraluminal vesicles would therefore reduce the protein at the limiting membrane of evolving MVBs. This could facilitate MBV mobility and docking events at the plasma membrane (62).

\subsubsection{The immediate and the delayed exosome pathway are differently influ- enced by cholesterol}

Cells release 'classical' exosomes that originate from intraluminal vesicles of the endosomal system and are released when MVBs fuse with the plasma membrane. One protein incorporated in exosomes via this process, is the proteolipid protein (PLP) (57). Besides using this delayed exosome pathway, cells also bud exosome-like vesicles immediately from the plasma membrane, without their previous cycling through the endosomal system. 
These immediately budding exosomes are released e.g. after infection with retroviruses, such as the human immunodeficiency virus (HIV) and the Moloney murine leukemia virus (MoMLV), which use the system for their propagation $(286 ; 30 ; 287)$. The viral exosomelike vesicles have a similar topology as 'classical' exosomes with their cytosolic membrane layer facing inside. Moreover, they appear to resemble 'classical' exosomes in lipid and protein composition, and in size (30). HIV and MoMLV produce the polyprotein Gag, which is essential for viral exosome-like vesicle formation. It was previously shown that infected $\mathrm{T}$ cells shed Gag-containing viral exosomes directly from the plasma membrane (286). Expression of Gag alone is already sufficient for the generation of non-infectious virus-like particles, without the need for other viral RNA and proteins (288).

In our experiments, we prepared exosomes from cell culture medium by a stepwise centrifugation protocol. This allows the removal of cell debris, larger microvesicles and membrane particles deriving, e.g., from the ER and Golgi apparatus. However, 'classical' exosomes that derive from the delayed pathway and exosome-like vesicles that are release via immediate budding from the plasma membrane are co-sedimented in our preparation. Western blot analysis of the $100,000 \mathrm{~g}$ pelleted material, which was further subjected to sucrose gradient ultracentrifugation, detected transfected MoMLV Gag-GFP protein at a density of $1.11 \mathrm{~g} / \mathrm{ml}$. This resembles the density of 'classical' exosomes. We therefore asked, whether cholesterol has an effect on the delayed exosome secretion pathway exclusively, or if the immediate plasma membrane-budding of exosome-like vesicles is also regulated by cholesterol levels.

In order to discriminate between the two different mechanisms of exosome release, we transfected Oli-neu cells with MoMLV Gag-GFP or PLP-myc and monitored their cellular localization and vesicular secretion from the cell. Immunofluorescene microscopy revealed co-localization of PLP-myc with endogenous flotillin-2 at the plasma membrane and in identical vesicular structures within the cytosol. In contrast, we could not observe any co-localization of flotillin-2 and Gag-GFP, but rather a separation of the two proteins into distinct domains. When we added cholesterol to the transfected cells, PLP-myc secretion via exosomes was markedly increased. The exosomal secretion of Gag-GFP protein, however, was not altered. These data indicate that the two discrete processes of exosome secretion are regulated by different factors. The pathway of immediate exosomelike vesicle budding, which can be used for retrovirus spreading (Gag), is not increased by high cholesterol levels. 
Interestingly, the authors Ikeda and Longnecker recently demonstrated that vesicular release of the latent membrane protein 2A (LMP2A) of Epstein-Barr virus (EBV) is enhanced after cholesterol-depletion from the plasma membrane via methyl- $\beta$-cyclodextrin. At the same time, cholesterol-depletion blocked LMP2A endocytosis and accumulated it at the plasma membrane (289). These findings underline our suggestion that although viral exosome-like vesicles can be sensitive to changes in cholesterol homeostasis, they behave differently than 'classical' exosomes.

We propose that those vesicles, which we found to be extensively secreted under high levels of cholesterol, are 'classical' exosomes, which derived from the delayed endocytosisdependent pathway that generates MVBs. Supporting this assumption, flotillin-2 mutagenized in its CRAC domains is largely impaired in endocytosis and can not be secreted via exosomes. Therefore, the delayed but not the immediate pathway of exosome release may contribute to the maintenance of cellular cholesterol homeostasis.

\subsection{Exosomal cholesterol release in NPC}

\subsubsection{Cholesterol release via exosomes is increased in NPC}

So far we discussed how cells adapt their exosome secretion to changes in cholesterol supply. We demonstrated that exosomal cholesterol release counteracts events that challenge the cell with depletion or oversupply of cholesterol. Proper structure and function of cells depend on a tight regulation of cholesterol. This is realized at the level of biosynthesis, uptake, metabolic conversion, and efflux of the lipid. Under physiological conditions, exosomes might provide a tool to fine tune cellular cholesterol levels. By this means, cells may reduce exosome-mediated cholesterol efflux under lipid starvation conditions. Cells, which contain superfluous cholesterol that remains unused for cellular processes, could increase exosomal cholesterol secretion to restore cholesterol homeostasis. Exosomes might therefore contribute to cellular cholesterol efflux.

This hypothesis becomes especially interesting under pathological conditions, when cells accumulate vast amounts of cholesterol, which can become toxic. In Niemann-Pick type C disease (NPC), the loss of functional NPC1 or NPC2 protein impairs free cholesterol translocation from late endosomes to the ER. As a result, the unesterified lipid is trapped and enriched in late endosomes from where it cannot leave the cell $(196 ; 197)$. This defect of a single step of cholesterol trafficking leads to a severe neuropathological phenotype. 
NPC patients suffer from extensive neurodegeneration and demyelination, causing major motor disabilities, seizures, dementia and eventually death (208).

As exosomes may have a role in maintaining cholesterol homeostasis, we investigated potential differences in exosomal cholesterol release by comparing wild-type cells with cells resembling the molecular phenotype of NPC. We first aimed at mimicking the disease by treating Oli-neu cells with the amphiphilic amine U18666A. This compound has been widely used to promote endosomal/lysosomal cholesterol accumulation (257; 231). Its mechanism of action is not fully understood, but it is proposed to bind to lysobisphophatidic acid (LBPA) (290), which might be a non-bilayer preferring lipid (291). LBPA possibly regulates the dynamic flow between the outer delimiting membrane and internal vesicles of late endosomes. U18666A is proposed to interfere in this process, subsequently impairing the normal membrane composition, which includes the proper distribution of NPC1 within late endosomal/lysosomal membranes (290). In our experiments, treatment of cells with U18666A induced a significant enhancement of exosomal cholesterol release. In contrast to this observation, cells did not increase exosome secretion when cholesterol was trapped in the ER by prevention of its esterification via an ACAT-inhibitor. This finding further supports the idea that cholesterol needs to undergo incorporation into the late endosomal system for its release via exosomes. It also underlines the specificity of exosomal secretion for a certain subpopulation of cholesterol in the cell.

Although U18666A has been used in various studies to mimic the NPC molecular phenotype, it it should be noted here that it has additional effects on cholesterol homeostasis. It was shown to inhibit cholesterol biosynthesis at the level of squalene epoxidase (292). It also can impair cholesterol's anterograde transport to axons in sympathetic neurons to a much higher extend than loss of functional NPC1 does (236). To verify the results obtained from our U18666A experiments, we mimicked NPC disease by a second approach using siRNA directed against NPC1. The down-regulation of NPC1 resulted in a similar increase in exosome release as compared to the application of U18666A. Moreover, the exosomal phenotype could be reproduced in NPC1-mutant Chinese hamster ovary (CHO) cells and in human fibroblasts derived from an NPC patient. Introduction of wild-type NPC1 in NPC1-mutant CHO cells reduced exosome secretion to wild-type levels.

We observed an enhancement of exosome release in several independent experiments on three different cell types. We therefore assume that the increase in MVBs and exsomal cholesterol secretion is a common feature of NPC cells. Recently, a Drosophila model of NPC1 was published, which mimics the pathology of human NPC patients (293). The 
flies displayed an age-progressive accumulation of unesterified cholesterol in brains, neurodegeneration, and motor defects, as well as a reduced life span. In support of our hypothesis, electron microscopy of fly brains revealed an accumulation of numerous MVBs in $\mathrm{NPC1}^{-/-}$fly neuropil and rhabdomers that progressed with age (293). In Purkinje cells of $\mathrm{NPC}^{-/-}$mice a similar accumulation of intracellular multilamellar bodies and membranous vesicles was found (294). Taken together, these data indicate that NPC cells enhance exosomal cholesterol release in an attempt to overcome the lipid's traffic block at late endosomes/lysosomes. By generating increased amounts of cholesterol-containing MVBs and exosomes, cells may possibly shuttle out parts of the lipid.

\subsubsection{Exocytosis of accumulating material in disease}

Since exosomes act as vehicles for molecule disposal (6), they might also facilitate the efflux of toxic accumulated material in various other diseases than NPC. In Niemann-Pick types A and B, which are characterized by deficiency in sphingomyelinase, cells store vast amounts of sphingomyelin in late endosomes/lysosomes (188). Importantly, sphingomyelin is also the second major accumulating product in NPC. It demands further investigation, whether exosomal secretion of sphingomyelin is similarly increased in the three NiemannPick diseases as cholesterol release is in NPC.

Another disorder that leads to lysosomal storage of lipids is metachromatic leukodystrophy (MLD). Patients suffering from MLD have a deficiency in arylsulfatase A, an enzyme catalyzing the first step of sulfatide degradation. Superfluous sulfatide is accumulated in lysosomes of oligodendrocytes and cells of the gall bladder and kidney (295). Similarly to NPC, this causes progressive demyelination and lethal neurological disabilities (296). Cultured primary kidney cells derived from an MLD mouse model enhanced plasma membrane-fusion of lysosomes to exocytose their contents upon $\mathrm{Ca}^{2+}$ stimulation. By this means the cells secreted a fraction of accumulated sulfatide into the culture medium (297). This process partially resembles the plasma membrane-fusion of MVBs and the release of exosomes.

A hallmark of Alzheimer's disease is the formation of $\mathrm{A} \beta$ peptides. It has been shown that $\beta$-cleavage of the amyloid precursor protein to $\mathrm{A} \beta$ occurs in early endosomes and is followed by routing the peptide to MVBs (17). Small amounts of $\mathrm{A} \beta$ can be released by exosomes (17). The pathology of Alzheimer's disease is classically thought to be caused by large aggregates of $\mathrm{A} \beta$ that assemble extracellularly (298). However, this model has recently been challenged by findings emphasizing the toxicity of intracellular $\mathrm{A} \beta(299 ; 300 ; 301)$. 
It might therefore be possible that cells secrete $\mathrm{A} \beta$ via exosomes in an attempt to shuttle out the toxic, accumulating material. This might also be true for the exosomal release of $\alpha$-synuclein and prion protein, the hallmarks of Parkinson's disease and Creutzfeldt-Jakob disease $(302 ; 84)$.

\subsubsection{How does exosomal cholesterol release affect the NPC phenotype?}

NPC cells enhance their exosomal cholesterol release. We propose this to be an attempt of the cells to partially overcome the late endosomal/lysosomal accumulation of cholesterol. However, it has to be mentioned here that the current knowledge about the molecular mechanism of NPC pathology is still inconclusive. Trapping of cholesterol itself might directly cause molecular toxicity. The polar lipid integrates into membranes and regulates their fluidity and permeability. Vast amounts of cholesterol could therefore impair the mobility of endosomal vesicles (247), and the proper function of lysosomes in degradation. On the other hand, retaining cholesterol at late endosomes and lysosomes leads to deprivation of the lipid in other cellular compartments. Although total levels of cholesterol are increased in NPC cells (231), its local absence may be the primary cause of the disease ('loss-of-function hypothesis'). Cholesterol is essential for a multitude of cellular processes. This includes formation of oxisterols in the ER (168), steroid hormone biosynthesis in mitochondria (173), and functions at the plasma membrane of distal neuronal axons $(231 ; 236 ; 237)$. Release of cholesterol via exosomes might partially reduce the lipid at late endosomes/lysosomes. However, this release will not compensate for NPC1/NPC2mediated intracellular cholesterol trafficking and, therefore, does not re-establish normal cholesterol levels in mitochondria, the ER or the plasma membrane. Conclusively, exosomes possibly do not ameliorate the NPC phenotype. They might instead even contribute to the pathomechanism of the disease. To clarify the significance of exosomal cholesterol release for the establishment of NPC pathology, one would have to experimentally manipulate exosome secretion in NPC cells. The severity cell toxicity could then be measured upon up- or down-regulation of exosome release. This is however difficult to realize since to date there are no activators or inhibitors known that act specifically and exclusively on the release of exosomes.

\subsubsection{Fate of exosomes}

In the future, it will be necessary to study exosomal cholesterol secretion in vivo to elucidate the fate of cholesterol-containing exosomes released by NPC-mutant cells. After 
secretion into the cellular environment, exosomes could either be taken up by neighboring cells or be disposed via body fluids.

Exosomes utilized for molecule disposal will have to be released via urine or taken up by cells specialized for degradation. It is likely that the latter is achieved by phagocytosis (81). To be recognized by phagocytes, target molecules destined for ingestion and degradation expose specific signals at their surface (303). Two of those signals are phosphatidylserine (PS) and MFGE8 - molecules readily found in exosomes $(284 ; 39)$. Exosomes further contain calcium-independent phospholipase A2 and capsase-3 $(304 ; 37)$. These proteins are involved in the generation of an epitope, which is integrated into the plasma membrane of cells during apoptosis and is recognized by $\operatorname{IgM}(305 ; 306)$.

After internalization, molecules are targeted to lysosomes where they are degraded. However, cells can not digest cholesterol. Instead, they convert it into cholesteryl esters, which are stored as cytosolic lipid droplets. It has recently been reported that monocytes take up T cell-derived exosomes via the PS receptor and accumulate free cholesterol and cholesteryl esters in their cytosol (307). Cholesterol storing monocytes/macrophages are involved in early events leading to arteriosclerosis (308). They internalize vast amounts of LDLcholesterol accumulated in blood vessels, and develop into foam cells (309). Interestingly, macrophages of $\mathrm{NPC}^{-/-}$mice accelerate the formation of arteriosclerotic lesions even under low serum cholesterol levels (310). It is therefore possible that increased amounts of cholesterol-containing exosomes in the blood are taken up by macrophages, and contribute to development of arteriosclerosis in NPC.

Exosomes not internalized by phagocytic cells could enter the blood circulation system and may be filtered through the kidney for excretion. The detection of exosomes in blood plasma and urine $(311 ; 312)$ is of great interest for the development of novel non-invasive biomarkers used in NPC disease monitoring.

\subsubsection{Exosomes as biomarkers for NPC}

We know of several diagnostic tools for the detection of NPC disorder. Besides the initial clinical assessment by a physician, this also includes neurophysiologic and neuroradiologic studies, histology of skin and liver biopsies, detection of foam cells, cell biological studies staining for free cholesterol by filipin or $\mathrm{BC} \theta$, and genetic testing (313). No effective treatment is currently known to heal NPC. However, after diagnosis of NPC, monitoring of disease progression and regular quantification of the degree of disabilities are important for the application of symptomatic therapies (314). Disease monitoring is further crucial 
in the development of new therapeutic strategies, e.g. for measuring drug efficacies or for assessing the patient's potential to respond to a certain therapy. To date, progression of NPC is mainly evaluated using disease-specific disability scales. These assign physiological and neurological symptoms to distinct classes and allow the calculation of a score representing the overall disease severity $(315 ; 316 ; 313)$. However, monitoring etiopathology at a molecular level, e.g. by determination of exosome release rates, might represent a more accurate method and would contribute to a reliable assessment of therapy efficacy.

Exosomes are released by virtually all cell types and can easily be prepared from body fluids, such as urine, blood plasma, and saliva $(312 ; 311 ; 317)$. Hence, they are very attractive as non-invasive biomarkers for different disorders. Numerous studies are currently undertaken to determine and validate possible exosomal biomarker proteins and RNA profiles that serve the diagnosis and monitoring of infectious diseases, renal dysfunctions, and various cancers $(318 ; 24 ; 319 ; 311 ; 320)$.

Urinal exosomes may provide us with a convenient marker to monitor disease progression and validate the efficacy of novel treatment strategies in NPC. Urine is available in large amounts and can be collected easily. Exosomes herein derive from renal epithelia cells facing the urinary space (321). During the course of NPC disease, urinal exosomes may undergo changes in their lipid and protein composition, or in their release rates. Any of these changes can be detected e.g. by Western blotting or mass spectrometry (321). These clinical assays involving relatively inexpensive techniques are valuable approaches, which might reduce costs in drug development and shorten the time required for drug testing. Before establishing those assays using urinary exosomes, certain barriers have to be overcome that may occur during collection, preservation, and analysis of samples. Quantitative comparison of urinary exosomes would make it necessary to determine the rate of exosome secretion. Urine should therefore be collected over a defined time period, e.g. for $24 \mathrm{~h}$. This is, however, impractical and inconvenient for patients, and does not deliver very reliable results (322). In a more practicable approach, urine is collected at one time point and concentrations of ubiquitous exosomal proteins such as alix and tsg-101 are normalized to concentrations of standard urine components, e.g. creatinine, inulin or other molecules that are filtered but not reabsorbed during urine production (321). If the samples can not be processed immediately after collection, degradation by proteolysis has to be prevented (323). This can be realized by addition of protease inhibitors to the collection vial or by storing the samples deep frozen until further processing (324). During preparation of exosomes from urine, cell debris and highly abundant proteins, such as Tamm-Horsfall 
protein and albumin in patients with proteinuria, have to be removed as they potentially obscure proteins of lower abundance in biochemical analysis $(24 ; 325)$. One also has to keep in mind that variations may occur in dependence of gender, age, diet, and circadian rhythm of the subjects. Taking this into account, a careful choice of controls is crucial for the successful application of urinary exosomes as biomarkers for drug efficacy and disease progression of NPC. 


\section{Chapter 5}

\section{Summary and Conclusions}

This work investigated exosome secretion as a novel mechanism for cholesterol egress. Cells release exosomes into their environment for a variety of purposes. One is the disposal of obsolete or superfluous molecules, which can potentially impair proper cell activities. To maintain their structural and functional integrity, cells crucially depend on a tight regulation of cholesterol homeostasis. This is realized at the levels of cholesterol synthesis, uptake, metabolism and efflux.

We first wanted to know whether exosomes can contribute to cholesterol homeostasis by facilitating its efflux from the cell. To approach this question, we studied exosome formation and release of the oligodendroglial precursor cell line Oli-neu, which readily secretes exosomes in quantifiable amounts. Exosomes were monitored via staining of the marker protein flotillin-2. We experimentally manipulated the cholesterol levels of Oli-neu cells by administration of cholesterol depleting drugs or induction of cholesterol enrichment. The first step of exosome generation involves invagination of the plasma membrane to form endosomes, which subsequently mature into MVBs. Cholesterol depletion retained flotillin2 at the plasma membrane. In contrast, enrichment in cholesterol promoted flotillin-2 incorporation into intracellular vesicular structures, where cholesterol co-localized with flotillin-2 and other markers of late endosomes and MVBs. We therefore hypothesize that exosome formation is enhanced under conditions of high cholesterol supply. We purified exosomes from cultured medium and found exosome release to be reduced upon cholesterol depletion and elevated by cholesterol enrichment. The potential of cells to adjust their exosome secretion to changes in cholesterol levels supports our hypothesis that exosomes facilitate cholesterol efflux.

Next, we were interested in the molecular mechanism by which exosomal cholesterol release is mediated. Flotillin-2 is known to associate to cholesterol-rich membrane microdomains 
at the plasma membrane. We reasoned that this association could be mediated by two cholesterol recognition/interaction amino acid consensus (CRAC) domains of flotillin-2. To assess their function, we introduced point mutations in flotillin's CRAC domains and monitored trafficking and exosomal release of these mutants. Mutation of the CRAC domains impaired both processes. We further asked whether flotillin-2 is involved in cholesterol secretion via exosomes. For this purpose flotillin-2 expression was down-regulated by siRNA. This treatment dramatically decreased the amount of exosome-secreted cholesterol. We therefore propose a model, in which flotillin-2 binds cholesterol at the plasma membrane via its CRAC domains, and thereby facilitates its endocytosis and recruitment to intraluminal vesicles of MVBs. After fusion of MVBs with the plasma membrane, cholesterol-containing exosomes are released.

Finally, we asked for the importance of exosomal cholesterol egress in a disease where cholesterol is accumulated in the cell. In Niemann-Pick type C disease (NPC), mutations in the NPC1 or NPC2 protein impair cholesterol trafficking from lysosomes to the ER and plasma membrane. The resulting storage of vast amounts of lysosomal cholesterol presumably causes progressive demyelination, neurodegeneration, and eventually death of NPC patients. To investigate exosome secretion in NPC, we mimicked the cellular phenotype of the disease by siRNA-mediated down-regulation of NPC1 or drug application. By these approaches, total exosome secretion and exosomal cholesterol release, respectively, were up-regulated. We could reproduce these results in human fibroblasts of NPC patients and in NPC1-mutant Chinese hamster ovary cells. Strikingly, exosome secretion of the latter was reduced to basal levels after transfection with wild type NPC1. We suggest that NPC cells enhance cholesterol release via exosomes in an attempt to partially bypass the late endosomal/lysosomal cholesterol traffic block. This enhancement in exosome secretion may be detectable and quantifiable in body fluids of NPC patients, opening a new possibility to monitor disease progression with exosome-based, longitudinal biomarkers.

In conclusion, we present a novel mechanism of cholesterol efflux, which depends on flotillin-2 and exosomes. It may contribute to the maintenance of cellular cholesterol homeostasis. 


\section{Bibliography}

[1] E. Cocucci, G. Racchetti, and J. Meldolesi. Shedding microvesicles: artefacts no more. Trends in cell biology, 19(2):43-51, 2009.

[2] M. Simons and G. Raposo. Exosomes - vesicular carriers for intercellular communication. Current opinion in cell biology, 21(4):575-581, 2009.

[3] C. Théry, M. Ostrowski, and E. Segura. Membrane vesicles as conveyors of immune responses. Nature Reviews Immunology, 9(8):581-593, 2009.

[4] B. T. Pan and R. M. Johnstone. Fate of the transferrin receptor during maturation of sheep reticulocytes in vitro: Selective externalization of the receptor. Cell, 33(3):967-977, 1983.

[5] C. Harding, J. Heuser, and P. Stahl. Receptor-mediated endocytosis of transferrin and recycling of the transferrin receptor in rat reticulocytes. Journal of Cell Biology, 97(2):329339, 1983.

[6] R. M. Johnstone, M. Adam, J. R. Hammond, L. Orr, and C. Turbide. Vesicle formation during reticulocyte maturation. association of plasma membrane activities with released vesicles (exosomes). Journal of Biological Chemistry, 262(19):9412-9420, 1987.

[7] S. Keller, M. P. Sanderson, A. Stoeck, and P. Altevogt. Exosomes: From biogenesis and secretion to biological function. Immunology letters, 107(2):102-108, 2006.

[8] G. Raposo, H. W. Nijman, W. Stoorvogel, R. Leijendekker, C. V. Harding, C. J. M. Melief, and H. J. Geuze. B lymphocytes secrete antigen-presenting vesicles. Journal of Experimental Medicine, 183(3):1161-1172, 1996.

[9] R. Wubbolts, R. S. Leckie, P. T. M. Veenhuizen, G. Schwarzmann, W. Möbius, J. Hoernschemeyer, J. W. Slot, H. J. Geuze, and W. Stoorvogel. Proteomic and biochemical analyses of human b cell-derived exosomes: Potential implications for their function and multivesicular body formation. Journal of Biological Chemistry, 278(13):10963-10972, 2003.

[10] C. Subra, K. Laulagnier, B. Perret, and M. Record. Exosome lipidomics unravels lipid sorting at the level of multivesicular bodies. Biochimie, 89(2):205-212, 2007. 
[11] H. Valadi, K. Ekström, A. Bossios, M. Sjöstrand, J. J. Lee, and J. O. Lötvall. Exosomemediated transfer of mrnas and micrornas is a novel mechanism of genetic exchange between cells. Nature cell biology, 9(6):654-659, 2007.

[12] V. Greco, M. Hannus, and S. Eaton. Argosomes: A potential vehicle for the spread of morphogens through epithelia. Cell, 106(5):633-645, 2001.

[13] C. Korkut, B. Ataman, P. Ramachandran, J. Ashley, R. Barria, N. Gherbesi, and V. Budnik. Trans-synaptic transmission of vesicular wnt signals through evi/wntless. Cell, 139(2):393404, 2009.

[14] D.G. Meckes Jr., K.H. Y. Shair, A.R. Marquitz, C. Kung, R.H. Edwards, and N. Raab-Traub. Human tumor virus utilizes exosomes for intercellular communication. PNAS, 107(47):20370$20375,2010$.

[15] A. J. Abusamra, Z. Zhong, X. Zheng, M. Li, T. E. Ichim, J. L. Chin, and W. P. Min. Tumor exosomes expressing fas ligand mediate cd8+t-cell apoptosis. Blood Cells, Molecules, and Diseases, 35(2):169-173, 2005.

[16] I. Porto-Carreiro, B. Février, S. Paquet, D. Vilette, and G. Raposo. Prions and exosomes: From prpc trafficking to prpsc propagation. Blood Cells, Molecules, and Diseases, 35(2):143$148,2005$.

[17] L. Rajendran, M. Honsho, T. R. Zahn, P. Keller, K. D. Geiger, P. Verkade, and K. Simons. Alzheimer's disease beta-amyloid peptides are released in association with exosomes. Proceedings of the National Academy of Sciences of the United States of America, 103(30):11172$11177,2006$.

[18] N. Blanchard, D. Lankar, F. Faure, A. Regnault, C. Dumont, G. Raposo, and C. Hivroz. Tcr activation of human $t$ cells induces the production of exosomes bearing the tcr/cd3/zeta complex. Journal of Immunology, 168(7):3235-3241, 2002.

[19] G. Raposo, D. Tenza, S. Mecheri, R. Peronet, C. Bonnerot, and C. Desaymard. Accumulation of major histocompatibility complex class ii molecules in mast cell secretory granules and their release upon degranulation. Molecular biology of the cell, 8(12):2631-2645, 1997.

[20] J. Fauré, G. Lachenal, M. Court, J. Hirrlinger, C. Chatellard-Causse, B. Blot, J. Grange, G. Schoehn, Y. Goldberg, V. Boyer, F. Kirchhoff, G. Raposo, J. Garin, and R. Sadoul. Exosomes are released by cultured cortical neurones. Molecular and Cellular Neuroscience, 31(4):642-648, 2006.

[21] E. M. Krämer-Albers, N. Bretz, S. Tenzer, C. Winterstein, W. Möbius, H. Berger, K. A. Nave, H. Schild, and J. Trotter. Oligodendrocytes secrete exosomes containing major myelin and 
stress-protective proteins: Trophic support for axons? Proteomics - Clinical Applications, 1(11):1446-1461, 2007.

[22] G. Van Niel, G. Raposo, C. Candalh, M. Boussac, R. Hershberg, N. Cerf-Bensussan, and M. Heyman. Intestinal epithelial cells secrete exosome-like vesicles. Gastroenterology, 121(2):337-349, 2001.

[23] M. P. Caby, D. Lankar, C. Vincendeau-Scherrer, G. Raposo, and C. Bonnerot. Exosomal-like vesicles are present in human blood plasma. International immunology, 17(7):879-887, 2005.

[24] T. Pisitkun, R. F. Shen, and M. A. Knepper. Identification and proteomic profiling of exosomes in human urine. Proceedings of the National Academy of Sciences of the United States of America, 101(36):13368-13373, 2004.

[25] L. J. Vella, D. L. V. Greenwood, R. Cappai, J. P. Y. Scheerlinck, and A. F. Hill. Enrichment of prion protein in exosomes derived from ovine cerebral spinal fluid. Veterinary immunology and immunopathology, 124(3-4):385-393, 2008.

[26] F. Andre, N. E. C. Schartz, M. Movassagh, C. Flament, P. Pautier, P. Morice, C. Pomel, C. Lhomme, B. Escudier, T. Le Chevalier, T. Tursz, S. Amigorena, G. Raposo, E. Angevin, and L. Zitvogel. Malignant effusions and immunogenic tumour-derived exosomes. Lancet, 360(9329):295-305, 2002.

[27] C. Théry, S. Amigorena, G. Raposo, and A. Clayton. Isolation and characterization of exosomes from cell culture supernatants and biological fluids. Current protocols in cell biology / editorial board, Juan S.Bonifacino et al., Chapter 3, 2006.

[28] L. Zitvogel, A. Regnault, A. Lozier, J. Wolfers, C. Flament, D. Tenza, P. RicciardiCastagnoli, G. Raposo, and S. Amigorena. Eradication of established murine tumors using a novel cell-free vaccine: Dendritic cell-derived exosomes. Nature medicine, 4(5):594-600, 1998.

[29] P. Gutwein, A. Stoeck, S. Riedle, D. Gast, S. Runz, T. P. Condon, A. Marmé, M. C. Phong, O. Linderkamp, A. Skorokhod, and P. Altevogt. Cleavage of 11 in exosomes and apoptotic membrane vesicles released from ovarian carcinoma cells. Clinical Cancer Research, 11(7):2492-2501, 2005.

[30] A. M. Booth, Y. Fang, J. K. Fallon, J. M. Yang, J. E. K. Hildreth, S. J. Gould, S. Sandefur, and V. Varthakavi. Exosomes and hiv gag bud from endosome-like domains of the t cell plasma membrane. Journal of Cell Biology, 172(6):923-935, 2006.

[31] J. W. Bess Jr., R. J. Gorelick, W. J. Bosche, L. E. Henderson, and L. O. Arthur. Microvesicles are a source of contaminating cellular proteins found in purified hiv-1 preparations. Virology, 230(1):134-144, 1997. 
[32] P. Rialland, D. Lankar, G. Raposo, C. Bonnerot, and P. Hubert. Bcr-bound antigen is targeted to exosomes in human follicular lymphoma b-cells. Biology of the Cell, 98(8):491$501,2006$.

[33] C. Théry, M. Boussac, P. Véron, P. Ricciardi-Castagnoli, G. Raposo, J. Garin, and S. Amigorena. Proteomic analysis of dendritic cell-derived exosomes: A secreted subcellular compartment distinct from apoptotic vesicles. Journal of Immunology, 166(12):7309-7318, 2001.

[34] A. Clayton, J. Court, H. Navabi, M. Adams, M. D. Mason, J. A. Hobot, G. R. Newman, and B. Jasani. Analysis of antigen presenting cell derived exosomes, based on immuno-magnetic isolation and flow cytometry. Journal of immunological methods, 247(1-2):163-174, 2001.

[35] C. Théry, L. Zitvogel, and S. Amigorena. Exosomes: Composition, biogenesis and function. Nature Reviews Immunology, 2(8):569-579, 2002.

[36] C. Hsu, Y. Morohashi, S. Yoshimura, N. Manrique-Hoyos, S. Jung, M. A. Lauterbach, M. Bakhti, M. Grønborg, W. Möbius, J. Rhee, F. A. Barr, and M. Simons. Regulation of exosome secretion by rab35 and its gtpase-activating proteins tbc1d10a-c. Journal of Cell Biology, 189(2):223-232, 2010.

[37] A. De Gassart, C. Géminard, B. Février, G. Raposo, and M. Vidal. Lipid raft-associated protein sorting in exosomes. Blood, 102(13):4336-4344, 2003.

[38] G. Wang, X. Zhou, Y. Bai, Z. Zhang, and D. Zhao. Cellular prion protein released on exosomes from macrophages binds to hsp70. Acta Biochimica et Biophysica Sinica, 42(5):345$350,2010$.

[39] C. Théry, A. Regnault, J. Garin, J. Wolfers, L. Zitvogel, P. Ricciardi-Castagnoli, G. Raposo, and S. Amigorena. Molecular characterization of dendritic cell-derived exosomes: Selective accumulation of the heat shock protein hsc73. Journal of Cell Biology, 147(3):599-610, 1999.

[40] K. Laulagnier, C. Motta, S. Hamdi, S. Roy, F. Fauvelle, J. F. Pageaux, T. Kobayashi, J. P. Salles, B. Perret, C. Bonnerot, and M. Record. Mast cell- and dendritic cell-derived display a specific lipid composition and an unusual membrane organization. Biochemical Journal, 380(1):161-171, 2004.

[41] H. F. G. Heijnen, A. E. Schiel, R. Fijnheer, H. J. Geuze, and J. J. Sixma. Activated platelets release two types of membrane vesicles: Microvesicles by surface shedding and exosomes derived from exocytosis of multivesicular bodies and alpha-granules. Blood, 94(11):3791$3799,1999$.

[42] Z. Chu, D. P. Witte, and X. Qi. Saposin c-lbpa interaction in late-endosomes/lysosomes. Experimental cell research, 303(2):300-307, 2005. 
[43] K. Denzer, M. Van Eijk, M. J. Kleijmeer, E. Jakobson, C. De Groot, and H. J. Geuze. Follicular dendritic cells carry mhc class ii-expressing microvesicles at their surface. Journal of Immunology, 165(3):1259-1265, 2000.

[44] E. D. Schwoebel and M. S. Moore. The control of gene expression by regulated nuclear transport. Essays in biochemistry, 36:105-113, 2000.

[45] R. C. Piper and J. P. Luzio. Late endosomes: Sorting and partitioning in multivesicular bodies. Traffic, 2(9):612-621, 2001.

[46] M. Babst. A protein's final escrt. Traffic, 6(1):2-9, 2005.

[47] M. Babst, B. Wendland, E. J. Estepa, and S. D. Emr. The vps4p aaa atpase regulates membrane association of a vps protein complex required for normal endosome function. EMBO Journal, 17(11):2982-2993, 1998.

[48] S. C. L. Yeo, L. Xu, J. Ren, V. J. Boulton, M. D. Wagle, C. Liu, G. Ren, P. Wong, R. Zahn, P. Sasajala, H. Yang, R. C. Piper, and A. L. Munn. Vps20p and vta1p interact with vps4p and function in multivesicular body sorting and endosomal transport in saccharomyces cerevisiae. Journal of cell science, 116(19):3957-3970, 2003.

[49] D. J. Katzmann, S. Sarkar, T. Chu, A. Audhya, and S. D. Emr. Multivesicular body sorting: Ubiquitin ligase rsp5 is required for the modification and sorting of carboxypeptidase s. Molecular biology of the cell, 15(2):468-480, 2004.

[50] M. Hochstrasser. Ubiquitin, proteasomes, and the regulation of intracellular protein degradation. Current opinion in cell biology, 7(2):215-223, 1995.

[51] D. J. Katzmann, M. Babst, and S. D. Emr. Ubiquitin-dependent sorting into the multivesicular body pathway requires the function of a conserved endosomal protein sorting complex, escrt-i. Cell, 106(2):145-155, 2001.

[52] C. Géminard, A. de Gassart, L. Blanc, and M. Vidal. Degradation of ap2 during reticulocyte maturation enhances binding of hsc70 and alix to a common site on tfr for sorting in exosomes. Traffic, 5(3):181-193, 2004.

[53] J. N. Hislop, A. Marley, and M. Von Zastrow. Role of mammalian vacuolar protein-sorting proteins in endocytic trafficking of a non-ubiquitinated g protein-coupled receptor to lysosomes. Journal of Biological Chemistry, 279(21):22522-22531, 2004.

[54] J. Wolfers, A. Lozier, G. Raposo, A. Regnault, C. Théry, C. Masurier, C. Flament, S. Pouzieux, F. Faure, T. Tursz, E. Angevin, S. Amigorena, and L. Zitvogel. Tumor-derived exosomes are a source of shared tumor rejection antigens for ctl cross-priming. Nature medicine, 7(3):297-303, 2001. 
[55] A. C. Theos, S. T. Truschel, D. Tenza, I. Hurbain, D. C. Harper, J. F. Berson, P. C. Thomas, G. Raposo, and M. S. Marks. A lumenal domain-dependent pathway for sorting to intralumenal vesicles of multivesicular endosomes involved in organelle morphogenesis. Developmental Cell, 10(3):343-354, 2006.

[56] Y. Fang, N. Wu, X. Gan, W. Yan, J. C. Morrell, and S. J. Gould. Higher-order oligomerization targets plasma membrane proteins and hiv gag to exosomes. PLoS biology, 5(6), 2007.

[57] K. Trajkovic, C. Hsu, S. Chiantia, L. Rajendran, D. Wenzel, F. Wieland, P. Schwille, B. Brügger, and M. Simons. Ceramide triggers budding of exosome vesicles into multivesicular endosomes. Science, 319(5867):1244-1247, 2008.

[58] T. Baumgart, S. T. Hess, and W. W. Webb. Imaging coexisting fluid domains in biomembrane models coupling curvature and line tension. Nature, 425(6960):821-824, 2003.

[59] M. Vidal, P. Mangeat, and D. Hoekstra. Aggregation reroutes molecules from a recycling to a vesicle-mediated secretion pathway during reticulocyte maturation. Journal of cell science, 110(16):1867-1877, 1997.

[60] A. Muntasell, A. C. Berger, and P. A. Roche. T cell-induced secretion of mhc class ii-peptide complexes on b cell exosomes. EMBO Journal, 26(19):4263-4272, 2007.

[61] F. M. Goñi and A. Alonso. Biophysics of sphingolipids i. membrane properties of sphingosine, ceramides and other simple sphingolipids. Biochimica et Biophysica Acta - Biomembranes, 1758(12):1902-1921, 2006.

[62] G. Van Niel, I. Porto-Carreiro, S. Simoes, and G. Raposo. Exosomes: A common pathway for a specialized function. Journal of Biochemistry, 140(1):13-21, 2006.

[63] M. Zöller. Tetraspanins: Push and pull in suppressing and promoting metastasis. Nature Reviews Cancer, 9(1):40-55, 2009.

[64] B. Février and G. Raposo. Exosomes: Endosomal-derived vesicles shipping extracellular messages. Current opinion in cell biology, 16(4):415-421, 2004.

[65] H. R. B. Pelham. Snares and the specificity of membrane fusion. Trends in cell biology, 11(3):99-101, 2001.

[66] Z. Guo, C. Turner, and D. Castle. Relocation of the t-snare snap-23 from lamellipodia-like cell surface projections regulates compound exocytosis in mast cells. Cell, 94(4):537-548, 1998.

[67] F. Paumet, J. Le Mao, S. Martin, T. Galli, B. David, U. Blank, and M. Roa. Soluble nsf attachment protein receptors (snares) in rbl-2h3 mast cells: Functional role of syntaxin 4 in 
exocytosis and identification of a vesicle- associated membrane protein 8-containing secretory compartment. Journal of Immunology, 164(11):5850-5857, 2000.

[68] T. Hibi, N. Hirashima, and M. Nakanishi. Rat basophilic leukemia cells express syntaxin-3 and vamp-7 in granule membranes. Biochemical and biophysical research communications, 271(1):36-41, 2000 .

[69] I. Martinez, S. Chakrabarti, T. Hellevik, J. Morehead, K. Fowler, and N. W. Andrews. Synaptotagmin vii regulates ca2+-dependent exocytosis of lysosomes in fibroblasts. Journal of Cell Biology, 148(6):1141-1149, 2000.

[70] S. K. Rao, C. Huynh, V. Proux-Gillardeaux, T. Galli, and N. W. Andrews. Identification of snares involved in synaptotagmin vii-regulated lysosomal exocytosis. Journal of Biological Chemistry, 279(19):20471-20479, 2004.

[71] M. C. Seabra, E. H. Mules, and A. N. Hume. Rab gtpases, intracellular traffic and disease. Trends in molecular medicine, 8(1):23-30, 2002.

[72] M. Ostrowski, N. B. Carmo, S. Krumeich, I. Fanget, G. Raposo, A. Savina, C. F. Moita, K. Schauer, A. N. Hume, R. P. Freitas, B. Goud, P. Benaroch, N. Hacohen, M. Fukuda, C. Desnos, M. C. Seabra, F. Darchen, S. Amigorena, L. F. Moita, and C. Thery. Rab27a and rab27b control different steps of the exosome secretion pathway. Nature cell biology, 12(1), 2010.

[73] A. Savina, M. Furlán, M. Vidal, and M. I. Colombo. Exosome release is regulated by a calcium-dependent mechanism in k562 cells. Journal of Biological Chemistry, 278(22):2008320090, 2003.

[74] A. Savina, C. M. Fader, M. T. Damiani, and M. I. Colombo. Rab11 promotes docking and fusion of multivesicular bodies in a calcium-dependent manner. Traffic, 6(2):131-143, 2005.

[75] E. J. Blott and G. M. Griffiths. Secretory lysosomes. Nature Reviews Molecular Cell Biology, $3(2): 122-131,2002$.

[76] R. J. Loomis, D. A. Holmes, A. Elms, P. A. Solski, C. J. Der, and L. Su. Citron kinase, a rhoa effector, enhances hiv-1 virion production by modulating exocytosis. Traffic, 7(12):1643$1653,2006$.

[77] V. Marshansky and M. Futai. The v-type h+-atpase in vesicular trafficking: targeting, regulation and function. Current opinion in cell biology, 20(4):415-426, 2008.

[78] S. Liégeois, A. Benedetto, J. M. Garnier, Y. Schwab, and M. Labouesse. The v0-atpase mediates apical secretion of exosomes containing hedgehog-related proteins in caenorhabditis elegans. Journal of Cell Biology, 173(6):949-961, 2006. 
[79] C. M. Fader, D. Sánchez, M. Furlán, and M. I. Colombo. Induction of autophagy promotes fusion of multivesicular bodies with autophagic vacuoles in k562 cells. Traffic, 9(2):230-250, 2008.

[80] H. Rabesandratana, J. P. Toutant, H. Reggio, and M. Vidal. Decay-accelerating factor (cd55) and membrane inhibitor of reactive lysis (cd59) are released within exosomes during in vitro maturation of reticulocytes. Blood, 91(7):2573-2580, 1998.

[81] L. Blanc, A. De Gassart, C. Géminard, P. Bette-Bobillo, and M. Vidal. Exosome release by reticulocytes - an integral part of the red blood cell differentiation system. Blood Cells, Molecules, and Diseases, 35(1):21-26, 2005.

[82] R. E. Waugh, J. B. Mckenney, R. G. Bauserman, D. M. Brooks, C. R. Valeri, and L. M. Snyder. Surface area and volume changes during maturation of reticulocytes in the circulation of the baboon. Journal of Laboratory and Clinical Medicine, 129(5):527-535, 1997.

[83] L. Da Costa, N. Mohandas, M. Sorette, M. J. Grange, G. Tchernia, and T. Cynober. Temporal differences in membrane loss lead to distinct reticulocyte features in hereditary spherocytosis and in immune hemolytic anemia. Blood, 98(10):2894-2899, 2001.

[84] B. Fevrier, D. Vilette, F. Archer, D. Loew, W. Faigle, M. Vidal, H. Laude, and G. Raposo. Cells release prions in association with exosomes. Proceedings of the National Academy of Sciences of the United States of America, 101(26):9683-9688, 2004.

[85] S. Utsugi-Kobukai, H. Fujimaki, C. Hotta, M. Nakazawa, and M. Minami. Mhe class imediated exogenous antigen presentation by exosomes secreted from immature and mature bone marrow derived dendritic cells. Immunology letters, 89(2-3):125-131, 2003.

[86] C. Admyre, S. M. Johansson, S. Paulie, and S. Gabrielsson. Direct exosome stimulation of peripheral human t cells detected by elispot. European journal of immunology, 36(7):1772$1781,2006$.

[87] P. K. Giri and J. S. Schorey. Exosomes derived from m. bovis bcg infected macrophages activate antigen-specific cd4+ and cd8+t cells in vitro and in vivo. PLoS ONE, 3(6), 2008.

[88] J. D. Walker, C. L. Maier, and J. S. Pober. Cytomegalovirus-infected human endothelial cells can stimulate allogeneic cd $4+$ memory t cells by releasing antigenic exosomes. Journal of Immunology, 182(3):1548-1559, 2009.

[89] G. Andreola, L. Rivoltini, C. Castelli, V. Huber, P. Perego, P. Deho, P. Squarcina, P. Accornero, F. Lozupone, L. Lugini, A. Stringaro, A. Molinari, G. Arancia, M. Gentile, G. Parmiani, and S. Fais. Induction of lymphocyte apoptosis by tumor cell secretion of fasl-bearing microvesicles. Journal of Experimental Medicine, 195(10):1303-1316, 2002. 
[90] C. Liu, S. Yu, K. Zinn, J. Wang, L. Zhang, Y. Jia, J. C. Kappes, S. Barnes, R. P. Kimberly, W. E. Grizzle, and H. G. Zhang. Murine mammary carcinoma exosomes promote tumor growth by suppression of nk cell function. Journal of Immunology, 176(3):1375-1385, 2006.

[91] E. Garcia, M. Pion, A. Pelchen-Matthews, L. Collinson, J. F. Arrighi, G. Blot, F. Leuba, J. M. Escola, N. Demaurex, M. Marsh, and V. Piguet. Hiv-1 trafficking to the dendritic cellt-cell infectious synapse uses a pathway of tetraspanin sorting to the immunological synapse. Traffic, 6(6):488-501, 2005.

[92] R. D. Wiley and S. Gummuluru. Immature dendritic cell-derived exosomes can mediate hiv-1 trans infection. Proceedings of the National Academy of Sciences of the United States of America, 103(3):738-743, 2006.

[93] L. J. Vella, R. A. Sharples, V. A. Lawson, C. L. Masters, R. Cappai, and A. F. Hill. Packaging of prions into exosomes is associated with a novel pathway of prp processing. Journal of Pathology, 211(5):582-590, 2007.

[94] H. Kokubo, C. A. Lemere, and H. Yamaguchi. Localization of flotillins in human brain and their accumulation with the progression of alzheimer's disease pathology. Neuroscience letters, 290(2):93-96, 2000.

[95] X. D. Cai, T. E. Golde, and S. G. Younkin. Release of excess amyloid beta protein from a mutant amyloid beta protein precursor. Science, 259(5094):514-516, 1993.

[96] H. Kokubo, T. C. Saido, N. Iwata, J. B. Helms, R. Shinohara, and H. Yamaguchi. Part of membrane-bound a-beta exists in rafts within senile plaques in tg2576 mouse brain. Neurobiology of aging, 26(4):409-418, 2005.

[97] T. Schulte, K. A. Paschke, U. Laessing, F. Lottspeich, and C. A. O. Stuermer. Reggie-1 and reggie-2, two cell surface proteins expressed by retinal ganglion cells during axon regeneration. Development, 124(2):577-587, 1997.

[98] P. E. Bickel, P. E. Scherer, J. E. Schnitzer, P. Oh, M. P. Lisanti, and H. F. Lodish. Flotillin and epidermal surface antigen define a new family of caveolae- associated integral membrane proteins. Journal of Biological Chemistry, 272(21):13793-13802, 1997.

[99] A. J. Edgar and J. M. Polak. Flotillin-1: Gene structure: cdna cloning from human lung and the identification of alternative polyadenylation signals. International Journal of Biochemistry and Cell Biology, 33(1):53-64, 2001.

[100] E. Rivera-Milla, C. A. O. Stuermer, and E. Málaga-Trillo. Ancient origin of reggie (flotillin), reggie-like, and other lipid-raft proteins: Convergent evolution of the spfh domain. Cellular and Molecular Life Sciences, 63(3):343-357, 2006. 
[101] G. H. H. Borner, D. J. Sherrier, T. Weimar, L. V. Michaelson, N. D. Hawkins, A. MacAskill, J. A. Napier, M. H. Beale, K. S. Lilley, and P. Dupree. Analysis of detergent-resistant membranes in arabidopsis. evidence for plasma membrane lipid rafts. Plant Physiology, 137(1):104-116, 2005.

[102] F. Galbiati, D. Volonté, J. S. Goltz, Z. Steele, J. Sen, J. Jurcsak, D. Stein, L. Stevens, and M. P. Lisanti. Identification, sequence and developmental expression of invertebrate flotillins from drosophila melanogaster. Gene, 210(2):229-237, 1998.

[103] D. Volonté, F. Galbiati, S. Li, K. Nishiyama, T. Okamoto, and M. P. Lisanti. Flotillins/cavatellins are differentially expressed in cells and tissues and form a hetero-oligomeric complex with caveolins in vivo: Characterization and epitope-mapping of a novel flotillin-1 monoclonal antibody probe. Journal of Biological Chemistry, 274(18):12702-12709, 1999.

[104] M. F. Langhorst, A. Reuter, and C. A. O. Stuermer. Scaffolding microdomains and beyond: The function of reggie/flotillin proteins. Cellular and Molecular Life Sciences, 62(1920):2228-2240, 2005.

[105] P. P. López-Casas and J. Del Mazo. Regulation of flotillin-1 in the establishment of nih-3t3 cell-cell interactions. FEBS letters, 555(2):223-228, 2003.

[106] D. M. Lang, S. Lommel, M. Jung, R. Ankerhold, B. Petrausch, U. Laessing, M. F. Wiechers, H. Plattner, and C. A. O. Stuermer. Identification of reggie-1 and reggie-2 as plasmamembrane-associated proteins which cocluster with activated gpi-anchored cell adhesion molecules in non-caveolar micropatches in neurons. Journal of neurobiology, 37(4):502$523,1998$.

[107] R. Gagescu, N. Demaurex, R. G. Parton, W. Hunziker, L. A. Huber, and J. Gruenberg. The recycling endosome of madin-darby canine kidney cells is a mildly acidic compartment rich in raft components. Molecular biology of the cell, 11(8):2775-2791, 2000.

[108] C. A. O. Stuermer, M. F. Langhorst, M. F. Wiechers, D. F. Legler, S. H. Von Hanwehr, A. H. Guse, and H. Plattner. Prpc capping in t cells promotes its association with the lipid raft proteins reggie-1 and reggie-2 and leads to signal transduction. FASEB Journal, 18(14):1731-1733, 2004.

[109] J. F. Dermine, S. Duclos, J. Garin, F. St.-Louis, S. Rea, R. G. Parton, and M. Desjardins. Flotillin-1-enriched lipid raft domains accumulate on maturing phagosomes. Journal of Biological Chemistry, 276(21):18507-18512, 2001.

[110] A. Santamaría, E. Castellanos, V. Gómez, P. Benedit, J. Renau-Piqueras, J. Morote, J. Reventós, T. M. Thomson, and R. Paciucci. Ptov1 enables the nuclear translocation 
and mitogenic activity of flotillin-1, a major protein of lipid rafts. Molecular and cellular biology, 25(5):1900-1911, 2005.

[111] H. Kokubo, J. B. Helms, Y. Ohno-Iwashita, Y. Shimada, Y. Horikoshi, and H. Yamaguchi. Ultrastructural localization of flotillin-1 to cholesterol-rich membrane microdomains, rafts, in rat brain tissue. Brain research, 965(1-2):83-90, 2003.

[112] K. Simons and D. Toomre. Lipid rafts and signal transduction. Nature Reviews Molecular Cell Biology, 1(1):31-39, 2000.

[113] D. T. Browman, M. B. Hoegg, and S. M. Robbins. The spfh domain-containing proteins: more than lipid raft markers. Trends in cell biology, 17(8):394-402, 2007.

[114] I. C. Morrow and R. G. Parton. Flotillins and the phb domain protein family: Rafts worms and anaesthetics. Traffic, 6(9):725-740, 2005.

[115] I. C. Morrow, S. Rea, S. Martin, I. A. Prior, R. Prohaska, J. F. Hancock, D. E. James, and R. G. Parton. Flotillin-1/reggie-2 traffics to surface raft domains via a novel golgiindependent pathway. identification of a novel membrane targeting domain and a role for palmitoylation. Journal of Biological Chemistry, 277(50):48834-48841, 2002.

[116] M. Bauer and L. Pelkmans. A new paradigm for membrane-organizing and -shaping scaffolds. FEBS letters, 580(23):5559-5564, 2006.

[117] C. Neumann-Giesen, B. Falkenbach, P. Beicht, S. Claasen, G. Lüers, C. A. O. Stuermer, V. Herzog, and R. Tikkanen. Membrane and raft association of reggie-1/flotillin-2: Role of myristoylation, palmitoylation and oligomerization and induction of filopodia by overexpression. Biochemical Journal, 378(2):509-518, 2004.

[118] W. T. Schroeder, S. Stewart-Galetka, S. Mandavilli, D. A. D. Parry, L. Goldsmith, and M. Duvic. Cloning and characterization of a novel epidermal cell surface antigen (esa). Journal of Biological Chemistry, 269(31):19983-19991, 1994.

[119] G. P. Solis, M. Hoegg, C. Munderloh, Y. Schrock, E. Malaga-Trillo, E. Rivera-Milla, and C. A. O. Stuermer. Reggie/flotillin proteins are organized into stable tetramers in membrane microdomains. Biochemical Journal, 403(2):313-322, 2007.

[120] T. Roitbak, Z. Surviladze, R. Tikkanen, and A. Wandinger-Ness. A polycystin multiprotein complex constitutes a cholesterol-containing signalling microdomain in human kidney epithelia. Biochemical Journal, 392(1):29-38, 2005.

[121] H. Li and V. Papadopoulos. Peripheral-type benzodiazepine receptor function in cholesterol transport. identification of a putative cholesterol recognition/interaction amino acid sequence and consensus pattern. Endocrinology, 139(12):4991-4997, 1998. 
[122] H. Li, Z. X. Yao, B. Degenhardt, G. Teper, and V. Papadopoulos. Cholesterol binding at the cholesterol recognition/interaction amino acid consensus (crac) of the peripheraltype benzodiazepine receptor and inhibition of steroidogenesis by an hiv tat-crac peptide. Proceedings of the National Academy of Sciences of the United States of America, 98(3):12671272, 2001.

[123] N. Jamin, J. M. Neumann, M. A. Ostuni, T. K. N. Vu, Z. X. Yao, S. Murail, J. C. Robert, C. Giatzakis, V. Papadopoulos, and J. . Lacapère. Characterization of the cholesterol recognition amino acid consensus sequence of the peripheral-type benzodiazepine receptor. Molecular Endocrinology, 19(3):588-594, 2005.

[124] R. M. Epand, B. G. Sayer, and R. F. Epand. Caveolin scaffolding region and cholesterol-rich domains in membranes. Journal of Molecular Biology, 345(2):339-350, 2005.

[125] P. Hazarika, N. Dham, P. Patel, M. Cho, D. Weidner, L. Goldsmith, and M. Duvic. Flotillin 2 is distinct from epidermal surface antigen (esa) and is associated with filopodia formation. Journal of cellular biochemistry, 75(1):147-159, 1999.

[126] C. Neumann-Giesen, I. Fernow, M. Amaddii, and R. Tikkanen. Role of egf-induced tyrosine phosphorylation of reggie-1/flotillin-2 in cell spreading and signaling to the actin cytoskeleton. Journal of cell science, 120(3):395-406, 2007.

[127] A. Kimura, C. A. Baumann, S. H. Chiang, and A. R. Saltiel. The sorbin homology domain: A motif for the targeting of proteins to lipid rafts. Proceedings of the National Academy of Sciences of the United States of America, 98(16):9098-9103, 2001.

[128] N. Kioka, K. Ueda, and T. Amachi. Vinexin, cap/ponsin, argbp2: A novel adaptor protein family regulating cytoskeletal organization and signal transduction. Cell structure and function, 27(1):1-7, 2002.

[129] C. A. Baumann, V. Ribon, M. Kanzaki, D. C. Thurmond, S. Mora, S. Shigematsu, P. E. Bickel, J. E. Pessin, and A. R. Saltiel. Cap defines a second signalling pathway required for insulin-stimulated glucose transport. Nature, 407(6801):202-207, 2000.

[130] J. Liu, S. M. DeYoung, M. Zhang, L. H. Dold, and A. R. Saltiel. The stomatin/prohibitin/flotillin/hflk/c domain of flotillin-1 contains distinct sequences that direct plasma membrane localization and protein interactions in 3t3-11 adipocytes. Journal of Biological Chemistry, 280(16):16125-16134, 2005.

[131] P. Mitra, X. Zheng, and M. P. Czech. Rnai-based analysis of cap, cbl, and crkii function in the regulation of glut4 by insulin. Journal of Biological Chemistry, 279(36):37431-37435, 2004. 
[132] C. Puri, D. Tosoni, R. Comai, A. Rabellino, D. Segat, F. Caneva, P. Luzzi, P. P. Di Fiore, and C. Tacchetti. Relationships between egfr signaling-competent and endocytosis-competent membrane microdomains. Molecular biology of the cell, 16(6):2704-2718, 2005.

[133] O. O. Glebov, N. A. Bright, and B. J. Nichols. Flotillin-1 defines a clathrin-independent endocytic pathway in mammalian cells. Nature cell biology, 8(1):46-54, 2006.

[134] M. Frick, N. A. Bright, K. Riento, A. Bray, C. Merrified, and B. J. Nichols. Coassembly of flotillins induces formation of membrane microdomains, membrane curvature, and vesicle budding. Current Biology, 17(13):1151-1156, 2007.

[135] M. Jiang, L. Jia, W. Jiang, X. Hu, H. Zhou, X. Gao, Z. Lu, and Z. Zhang. Protein disregulation in red blood cell membranes of type 2 diabetic patients. Biochemical and biophysical research communications, 309(1):196-200, 2003.

[136] K. Bloch. The structure of cholesterol and of the bile acids. Trends in biochemical sciences, $7(9): 334-336,1982$.

[137] F. R. Maxfield and I. Tabas. Role of cholesterol and lipid organization in disease. Nature, 438(7068):612-621, 2005.

[138] J. M. Dietschy and S. D. Turley. Cholesterol metabolism in the central nervous system during early development and in the mature animal. Journal of lipid research, 45(8):13751397, 2004.

[139] J. Vaya and H. M. Schipper. Oxysterols, cholesterol homeostasis, and alzheimer disease. Journal of neurochemistry, 102(6):1727-1737, 2007.

[140] Y. A. Ioannou. Multidrug permeases and subcellular cholesterol transport. Nature Reviews Molecular Cell Biology, 2(9):657-668, 2001.

[141] D. E. Vance and H. Van Den Bosch. Cholesterol in the year 2000. Biochimica et Biophysica Acta - Molecular and Cell Biology of Lipids, 1529(1-3):1-8, 2000.

[142] H. Jurevics and P. Morell. Cholesterol for synthesis of myelin is made locally, not imported into brain. Journal of neurochemistry, 64(2):895-901, 1995.

[143] P. J. Espenshade and A. L. Hughes. Regulation of sterol synthesis in eukaryotes. Annual Review of Genetics, 41:401-427, 2007.

[144] A. Radhakrishnan, L. P. Sun, H. J. Kwon, M. S. Brown, and J. L. Goldstein. Direct binding of cholesterol to the purified membrane region of scap: Mechanism for a sterol-sensing domain. Molecular cell, 15(2):259-268, 2004. 
[145] A. Nohturfft, D. Yabe, J. L. Goldstein, M. S. Brown, and P. J. Espenshade. Regulated step in cholesterol feedback localized to budding of scap from er membranes. Cell, 102(3):315-323, 2000 .

[146] M. S. Brown and J. L. Goldstein. The srebp pathway: Regulation of cholesterol metabolism by proteolysis of a membrane-bound transcription factor. Cell, 89(3):331-340, 1997.

[147] J. L. Goldstein, R. A. DeBose-Boyd, and M. S. Brown. Protein sensors for membrane sterols. Cell, 124(1):35-36, 2006.

[148] M. S. Brown and J. L. Goldstein. A receptor-mediated pathway for cholesterol homeostasis. Science, 232(4746):34-47, 1986.

[149] S. Sugii, P. C. Reid, N. Ohgami, H. Du, and T. Y. Chang. Distinct endosomal compartments in early trafficking of low density lipoprotein-derived cholesterol. Journal of Biological Chemistry, 278(29):27180-27189, 2003.

[150] K. M. Wojtanik and L. Liscum. The transport of low density lipoprotein-derived cholesterol to the plasma membrane is defective in npc1 cells. Journal of Biological Chemistry, 278(17):14850-14856, 2003.

[151] S. D. Turley, D. K. Burns, C. R. Rosenfeld, and J. M. Dietschy. Brain does not utilize low density lipoprotein-cholesterol during fetal and neonatal development in the sheep. Journal of lipid research, 37(9):1953-1961, 1996.

[152] R. E. Pitas, J. K. Boyles, S. H. Lee, D. Hui, and K. H. Weisgraber. Lipoproteins and their receptors in the central nervous system. characterization of the lipoproteins in cerebrospinal fluid and identification of apolipoprotein b,e(ldl) receptors in the brain. Journal of Biological Chemistry, 262(29):14352-14360, 1987.

[153] N. Wang, D. Lan, W. Chen, F. Matsuura, and A. R. Tall. Atp-binding cassette transporters g1 and g4 mediate cellular cholesterol efflux to high-density lipoproteins. Proceedings of the National Academy of Sciences of the United States of America, 101(26):9774-9779, 2004.

[154] T. Rothe and H. W. Muller. Uptake of endoneurial lipoprotein into schwann cells and sensory neurons is mediated by low density lipoprotein receptors and stimulated after axonal injury. Journal of neurochemistry, 57(6):2016-2025, 1991.

[155] E. B. Neufeld, A. M. Cooney, J. Pitha, E. A. Dawidowicz, N. K. Dwyer, P. G. Pentchev, and E. Joan Blanchette-Mackie. Intracellular trafficking of cholesterol monitored with a cyclodextrin. Journal of Biological Chemistry, 271(35):21604-21613, 1996. 
[156] I. Tabas, W. J. Rosoff, and G. C. Boykow. Acyl coenzyme a:cholesterol acyl transferase in macrophages utilizes a cellular pool of cholesterol oxidase-accessible cholesterol as substrate. Journal of Biological Chemistry, 263(3):1266-1272, 1988.

[157] L. Pelkmans, J. Kartenbeck, and A. Helenius. Caveolar endocytosis of simian virus 40 reveals a new two-step vesicular-transport pathway to the er. Nature cell biology, 3(5):473-483, 2001.

[158] T.Y. Chang, C. C. Y. Chang, N. Ohgami, and Y. Yamauchi. Cholesterol sensing, trafficking, and esterification. 22:129-157, 2006.

[159] Y. Lange, J. Ye, and T. L. Steck. Circulation of cholesterol between lysosomes and the plasma membrane. Journal of Biological Chemistry, 273(30):18915-18922, 1998.

[160] A. A. Spector, S. N. Mathur, and T. L. Kaduce. Role of acylcoenzyme a: Cholesterol o-acyltransferase in cholesterol metabolism. Progress in lipid research, 18(1):31-53, 1979.

[161] J. Liu, C. C. Y. Chang, E. J. Westover, D. F. Covey, and T. Y. Chang. Investigating the allosterism of acyl-coa:cholesterol acyltransferase (acat) by using various sterols: In vitro and intact cell studies. Biochemical Journal, 391(2):389-397, 2005.

[162] M. S. Brown, Y. K. Ho, and J. L. Goldstein. The cholesteryl ester cycle in macrophage foam cells. continual hydrolysis and re-esterification of cytoplasmic cholesteryl esters. Journal of Biological Chemistry, 255(19):9344-9352, 1980.

[163] Y. Yamauchi, C. C. Y. Chang, M. Hayashi, S. Abe-Dohmae, P. C. Reid, T. Y. Chang, and S. Yokoyama. Intracellular cholesterol mobilization involved in the abca1/ apolipoproteinmediated assembly of high density lipoprotein in fibroblasts. Journal of lipid research, 45(10):1943-1951, 2004.

[164] J. F. Strauss III, T. Kishida, L. K. Christenson, T. Fujimoto, and H. Hiroi. Start domain proteins and the intracellular trafficking of cholesterol in steroidogenic cells. Molecular and cellular endocrinology, 202(1-2):59-65, 2003.

[165] J. Reitz, K. Gehrig-Burger, J. F. Strauss III, and G. Gimpl. Cholesterol interaction with the related steroidogenic acute regulatory lipid-transfer (start) domains of star (stard1) and mln64 (stard3). FEBS Journal, 275(8):1790-1802, 2008.

[166] Y. J. Im, S. Raychaudhuri, W. A. Prinz, and J. H. Hurley. Structural mechanism for sterol sensing and transport by osbp-related proteins. Nature, 437(7055):154-158, 2005.

[167] A. Choudhury, M. Dominguez, V. Puri, D. K. Sharma, K. Narita, C. L. Wheatley, D. L. Marks, and R. E. Pagano. Rab proteins mediate golgi transport of caveola-internalized glycosphingolipids and correct lipid trafficking in niemann-pick c cells. Journal of Clinical Investigation, 109(12):1541-1550, 2002. 
[168] T. Li, E. Owsley, M. Matozel, P. Hsu, C. M. Novak, and J. Y. L. Chiang. Transgenic expression of cholesterol 7alpha-hydroxylase in the liver prevents high-fat diet-induced obesity and insulin resistance in mice. Hepatology, 52(2), 2010.

[169] E. G. Lund, C. Xie, T. Kotti, S. D. Turley, J. M. Dietschy, and D. W. Russell. Knockout of the cholesterol 24-hydroxylase gene in mice reveals a brain-specific mechanism of cholesterol turnover. Journal of Biological Chemistry, 278(25):22980-22988, 2003.

[170] A. J. Brown, L. Sun, J. D. Feramisco, M. S. Brown, and J. L. Goldstein. Cholesterol addition to er membranes alters conformation of scap, the srebp escort protein that regulates cholesterol metabolism. Molecular cell, 10(2):237-245, 2002.

[171] X. Fu, J. G. Menke, Y. Chen, G. Zhou, K. L. MacNaul, S. D. Wright, C. P. Sparrow, and E. G. Lund. 27-hydroxycholesterol is an endogenous ligand for liver $\mathrm{x}$ receptor in cholesterolloaded cells. Journal of Biological Chemistry, 276(42):38378-38387, 2001.

[172] T. Hauet, Z. X. Yao, H. S. Bose, C. T. Wall, Z. Han, W. Li, D. B. Hales, W. L. Miller, M. Culty, and V. Papadopoulos. Peripheral-type benzodiazepine receptor-mediated action of steroidogenic acute regulatory protein on cholesterol entry into leydig cell mitochondria. Molecular Endocrinology, 19(2):540-554, 2005.

[173] S. Sugano, R. Miura, and N. Morishima. Identification of intermediates in the conversion of cholesterol to pregnenolone with a reconstituted cytochrome p-450(scc) system: Accumulation of the intermediate modulated by the adrenodoxin level. Journal of Biochemistry, 120(4):780-787, 1996.

[174] G. H. Rothblat and M. C. Phillips. High-density lipoprotein heterogeneity and function in reverse cholesterol transport. Current opinion in lipidology, 21(3):229-238, 2010.

[175] M. Kawano, T. Miida, C. J. Fielding, and P. E. Fielding. Quantitation of pre beta-hdldependent and nonspecific components of the total efflux of cellular cholesterol and phospholipid. Biochemistry, 32(19):5025-5028, 1993.

[176] E. J. Smart, Y. Ying, W. C. Donzell, and R. G. W. Anderson. A role for caveolin in transport of cholesterol from endoplasmic reticulum to plasma membrane. Journal of Biological Chemistry, 271(46), 1996.

[177] M. De La Llera-Moya, M. A. Connelly, D. Drazul, S. M. Klein, E. Favari, P. G. Yancey, D. L. Williams, and G. H. Rothblat. Scavenger receptor class b type i affects cholesterol homeostasis by magnifying cholesterol flux between cells and hdl. Journal of lipid research, 42(12):1969-1978, 2001.

[178] J. F. Oram and A. M. Vaughan. Abca1-mediated transport of cellular cholesterol and phospholipids to hbl apolipoproteins. Current opinion in lipidology, 11(3):253-260, 2000. 
[179] S. Azhar and E. Reaven. Scavenger receptor class bi and selective cholesteryl ester uptake: Partners in the regulation of steroidogenesis. Molecular and cellular endocrinology, 195(12):1-26, 2002.

[180] I. Björkhem, D. Lütjohann, O. Breuer, A. Sakinis, and $\AA$ Wennmalm. Importance of a novel oxidative mechanism for elimination of brain cholesterol. turnover of cholesterol and 24(s)-hydroxycholesterol in rat brain as measured with 1802 techniques in vivo and in vitro. Journal of Biological Chemistry, 272(48):30178-30184, 1997.

[181] S. Meaney, K. Bodin, U. Diczfalusy, and I. Björkhem. On the rate of translocation in vitro and kinetics in vivo of the major oxysterols in human circulation: Critical importance of the position of the oxygen function. Journal of lipid research, 43(12):2130-2135, 2002.

[182] J. E. Vance, H. Hayashi, and B. Karten. Cholesterol homeostasis in neurons and glial cells. Seminars in Cell and Developmental Biology, 16(2):193-212, 2005.

[183] A. Brooks-Wilson, M. Marcil, S. M. Clee, L. H. Zhang, K. Roomp, M. Van Dam, L. Yu, C. Brewer, J. A. Collins, H. O. F. Molhuizen, O. Loubser, B. F. F. Ouelette, K. Fichter, K. J. D. Ashbourne-Excoffon, C. W. Sensen, S. Scherer, S. Mott, M. Denis, D. Martindale, J. Frohlich, K. Morgan, B. Koop, S. Pimstone, J. J. P. Kastelein, J. Genest Jr., and M. R. Hayden. Mutations in abc1 in tangier disease and familial high-density lipoprotein deficiency. Nature genetics, 22(4):336-345, 1999.

[184] S. Rust, M. Rosier, H. Funke, J. Real, Z. Amoura, J. C. Piette, J. F. Deleuze, H. B. Brewer, N. Duverger, P. Denèfle, and G. Assmann. Tangier disease is caused by mutations in the gene encoding atp-binding cassette transporter 1. Nature genetics, 22(4):352-355, 1999.

[185] A. M. Saunders, W. J. Strittmatter, D. Schmechel, P. H. St. George-Hyslop, M. A. PericakVance, S. H. Joo, B. L. Rosi, J. F. Gusella, D. R. Crapper-MacLachlan, M. J. Alberts, C. Hulette, B. Crain, D. Goldgaber, and A. D. Roses. Association of apolipoprotein e allele epsilon 4 with late-onset familial and sporadic alzheimer's disease. Neurology, 43(8):1467$1472,1993$.

[186] L. Bertram, M. B. McQueen, K. Mullin, D. Blacker, and R. E. Tanzi. Systematic metaanalyses of alzheimer disease genetic association studies: The alzgene database. Nature genetics, 39(1):17-23, 2007.

[187] A. C. Crocker. The cerebral defect in tay-sachs disease and niemann-pick disease. Journal of neurochemistry, 7:69-80, 1961.

[188] R. O. Brady, J. N. Kanfer, M. B. Mock, and D. S. Fredrickson. The metabolism of sphingomyelin. ii. evidence of an enzymatic deficiency in niemann-pick disease. Proceedings of the National Academy of Sciences of the United States of America, 55(2):366-369, 1966. 
[189] E. H. Schuchman, M. Suchi, T. Takahashi, K. Sandhoff, and R. J. Desnick. Human acid sphingomyelinase: Isolation, nucleotide sequence, and expression of the full-length and alternatively spliced cdnas. Journal of Biological Chemistry, 266(13):8531-8539, 1991.

[190] P. B. Schneider and E. P. Kennedy. Sphingomyelinase in normal human spleens and in spleens from subjects with niemann-pick disease. Journal of lipid research, 8(3):202-209, 1967.

[191] P. G. Pentchev, A. D. Boothe, and H. S. Kurth. A genetic storage disorder in balb/c mice with a metabolic block in esterification of exogenous cholesterol. Journal of Biological Chemistry, 259(9):5784-5791, 1984.

[192] S. K. Loftus, J. A. Morris, E. D. Carstea, J. Z. Gu, C. Cummings, A. Brown, J. Ellison, K. Ohno, M. A. Rosenfeld, D. A. Tagle, P. G. Pentchev, and W. J. Pavan. Murine model of niemann-pick c disease: Mutation in a cholesterol homeostasis gene. Science, 277(5323):232$235,1997$.

[193] E. J. T. Winsor and J. P. Welch. Genetic and demographic aspects of nova scotia niemannpick disease (type d). American Journal of Human Genetics, 30(5):530-538, 1978.

[194] W. L. Greer, D. C. Riddell, D. M. Byers, J. P. Welch, G. S. Girouard, S. M. Sparrow, T. L. Gillan, and P. E. Neumann. Linkage of niemann-pick disease type $\mathrm{d}$ to the same region of human chromosome 18 as niemann-pick disease type c. American Journal of Human Genetics, 61(1):139-142, 1997.

[195] P. G. Pentchev, M. T. Vanier, K. Suzuki, and M. C. Patterson. Niemann-pick disease type c: A cellular cholesterol lipidosis. The Metabolic and Molecular Bases of Inherited Disease, pages 2625-2639, 1995.

[196] J. Sokol, E. J. Blanchette-Mackie, H. S. Kruth, N. K. Dwyer, L. M. Amende, J. D. Butler, E. Robinson, S. Patel, R. O. Brady, M. E. Comly, M. T. Vanier, and P. G. Pentchev. Type c niemann-pick disease. lysosomal accumulation and defective intracellular mobilization of low density lipoprotein cholesterol. Journal of Biological Chemistry, 263(7):3411-3417, 1988.

[197] L. Liscum, R. M. Ruggiero, and J. R. Faust. The intracellular transport of low density lipoprotein-derived cholesterol is defective in niemann-pick type c fibroblasts. Journal of Cell Biology, 108(5):1625-1636, 1989.

[198] M. T. Vanier and K. Suzuki. Recent advances in elucidating niemann-pick c disease. Brain Pathology, 8(1):163-174, 1998.

[199] M. T. Vanier and G. Millat. Niemann-pick disease type c. Clinical genetics, 64(4):269-281, 2003. 
[200] G. Millat, C. Marçais, M. A. Rafi, T. Yamamoto, J. A. Morris, P. G. Pentchev, K. Ohno, D. A. Wenger, and M. T. Vanier. Niemann-pick c1 disease: The i1061t substitution is a frequent mutant allele in patients of western european descent and correlates with a classic juvenile phenotype. American Journal of Human Genetics, 65(5):1321-1329, 1999.

[201] E. D. Carstea, M. H. Polymeropoulos, C. C. Parker, S. D. Detera-Wadleigh, R. R. O'Neill, M. C. Patterson, E. Goldin, H. Xiao, R. E. Straub, M. T. Vanier, R. O. Brady, and P. G. Pentchev. Linkage of niemann-pick disease type $\mathrm{c}$ to human chromosome 18. Proceedings of the National Academy of Sciences of the United States of America, 90(5):2002-2004, 1993.

[202] W. L. Greer, D. C. Riddell, T. L. Gillan, G. S. Girouard, S. M. Sparrow, D. M. Byers, M. J. Dobson, and P. E. Neumann. The nova scotia (type d) form of niemann-pick disease is caused by a g3097 $\rightarrow$ t transversion in npc1. American Journal of Human Genetics, 63(1):52$54,1998$.

[203] C. D. Pacheco and A. P. Lieberman. The pathogenesis of niemann-pick type c disease: a role for autophagy? Expert reviews in molecular medicine, 10:e26, 2008.

[204] T. Kobayashi, M. H. Beuchat, M. Lindsay, S. Frias, R. D. Palmiter, H. Sakuraba, R. G. Parton, and J. Gruenberg. Late endosomal membranes rich in lysobisphosphatidic acid regulate cholesterol transport. Nature cell biology, 1(2):113-118, 1999.

[205] Y. Watanabe, S. Akaboshi, G. Ishida, T. Takeshima, T. Yano, M. Taniguchi, K. Ohno, and K. Nakashima. Increased levels of gm2 ganglioside in fibroblasts from a patient with juvenile niemann-pick disease type c. Brain and Development, 20(2):95-97, 1998.

[206] M. Zervas, K. Dobrenis, and S. U. Walkley. Neurons in niemann-pick disease type c accumulate gangliosides as well as unesterified cholesterol and undergo dendritic and axonal alterations. Journal of neuropathology and experimental neurology, 60(1):49-64, 2001.

[207] D. Te Vruchte, E. Lloyd-Evans, R. J. Veldman, D. C. A. Neville, R. A. Dwek, F. M. Platt, W. J. Van Blitterswijk, and D. J. Sillences. Accumulation of glycosphingolipids in niemannpick c disease disrupts endosomal transport. Journal of Biological Chemistry, 279(25):26167$26175,2004$.

[208] Y. Higashi, S. Murayama, P. G. Pentchev, and K. Suzuki. Cerebellar degeneration in the niemann-pick type c mouse. Acta Neuropathologica, 85(2):175-184, 1993.

[209] O. Schofer, B. Mischo, W. Püschel, K. Harzer, and M. T. Vanier. Early-lethal pulmonary form of niemann-pick type c disease belonging to a second, rare genetic complementation group. European journal of pediatrics, 157(1):45-49, 1998. 
[210] M. Zarowski, B. Steinborn, B. Gurda, L. Dvorakova, H. Vlaskova, and S. V. Kothare. Treatment of cataplexy in niemann-pick disease type c with the use of miglustat. European Journal of Paediatric Neurology, 2010. Article in Press.

[211] M. E. Higgins, J. P. Davies, F. W. Chen, and Y. A. Ioannou. Niemann-pick c1 is a late endosome-resident protein that transiently associates with lysosomes and the trans-golgi network. Molecular genetics and metabolism, 68(1):1-13, 1999.

[212] J. P. Davies and Y. A. Ioannou. Topological analysis of niemann-pick c1 protein reveals that the membrane orientation of the putative sterol-sensing domain is identical to those of 3-hydroxy-3-methylglutaryl-coa reductase and sterol regulatory element binding protein cleavage-activating protein. Journal of Biological Chemistry, 275(32):24367-24374, 2000.

[213] E. E. Millard, S. E. Gale, N. Dudley, J. Zhang, J. E. Schaffer, and D. S. Ory. The sterolsensing domain of the niemann-pick c1 (npc1) protein regulates trafficking of low density lipoprotein cholesterol. Journal of Biological Chemistry, 280(31):28581-28590, 2005.

[214] C. Scott and Y. A. Ioannou. The npc1 protein: Structure implies function. Biochimica et Biophysica Acta - Molecular and Cell Biology of Lipids, 1685(1-3):8-13, 2004.

[215] E. D. Carstea, J. A. Morris, K. G. Coleman, S. K. Loftus, D. Zhang, C. Cummings, J. Gu, M. A. Rosenfeld, W. J. Pavan, D. B. Krizman, J. Nagle, M. H. Polymeropoulos, S. L. Sturley, Y. A. Ioannou, M. E. Higgins, M. Comly, A. Cooney, A. Brown, C. R. Kaneski, E. J. Blanchette-Mackie, N. K. Dwyer, E. B. Neufeld, T. Y. Chang, L. Liscum, J. F. Strauss III, K. Ohno, M. Zeigler, R. Carmi, J. Sokol, D. Markie, R. R. O'Neill, O. P. Van Diggelen, M. Elleder, M. C. Patterson, R. O. Brady, M. T. Vanier, P. G. Pentchev, and D. A. Tagle. Niemann-pick c1 disease gene: Homology to mediators of cholesterol homeostasis. Science, 277(5323):228-231, 1997.

[216] N. Friedland, H. L. Liou, P. Lobel, and A. M. Stock. Structure of a cholesterol-binding protein deficient in niemann-pick type $\mathrm{c} 2$ disease. Proceedings of the National Academy of Sciences of the United States of America, 100(5):2512-2517, 2003.

[217] S. Naureckiene, D. E. Sleat, H. Lacklan, A. Fensom, M. T. Vanier, R. Wattiaux, M. Jadot, and P. Lobel. Identification of he1 as the second gene of niemann-pick c disease. Science, 290(5500):2298-2301, 2000.

[218] M. T. Vanier and G. Millat. Structure and function of the npc2 protein. Biochimica et Biophysica Acta - Molecular and Cell Biology of Lipids, 1685(1-3):14-21, 2004.

[219] R. E. Infante, A. Radhakrishnan, L. Abi-Mosleh, L. N. Kinch, M. L. Wang, N. V. Grishin, J. L. Goldstein, and M. S. Brown. Purified npc1 protein ii. localization of sterol binding to 
a 240-amino acid soluble luminal loop. Journal of Biological Chemistry, 283(2):1064-1075, 2008.

[220] R. E. Infante, M. L. Wang, A. Radhakrishnan, J. K. Hyock, M. S. Brown, and J. L. Goldstein. $\mathrm{Npc} 2$ facilitates bidirectional transfer of cholesterol between npc1 and lipid bilayers, a step in cholesterol egress from lysosomes. Proceedings of the National Academy of Sciences of the United States of America, 105(40):15287-15292, 2008.

[221] R. E. Infante, M. L. Wang, A. Radhakrishnan, J. K. Hyock, M. S. Brown, and J. L. Goldstein. $\mathrm{Npc} 2$ facilitates bidirectional transfer of cholesterol between npc1 and lipid bilayers, a step in cholesterol egress from lysosomes. Proceedings of the National Academy of Sciences of the United States of America, 105(40):15287-15292, 2008.

[222] J. P. Liu, Y. Tang, S. Zhou, B. H. Toh, C. McLean, and H. Li. Cholesterol involvement in the pathogenesis of neurodegenerative diseases. Molecular and Cellular Neuroscience, 43(1):33-42, 2010.

[223] H. J. Kwon, L. Abi-Mosleh, M. L. Wang, J. Deisenhofer, J. L. Goldstein, M. S. Brown, and R. E. Infante. Structure of n-terminal domain of npc1 reveals distinct subdomains for binding and transfer of cholesterol. Cell, 137(7):1213-1224, 2009.

[224] D. E. Sleat, J. A. Wiseman, M. El-Banna, S. M. Price, L. Verot, M. M. Shen, G. S. Tint, M. T. Vanier, S. U. Walkley, and P. Lobel. Genetic evidence for nonredundant functional cooperativity between npc1 and npc2 in lipid transport. Proceedings of the National Academy of Sciences of the United States of America, 101(16):5886-5891, 2004.

[225] R. P. Erickson, W. S. Garver, F. Camargo, G. S. Hossain, and R. A. Heidenreich. Pharmacological and genetic modifications of somatic cholesterol do not substantially alter the course of cns disease in niemann-pick c mice. Journal of inherited metabolic disease, 23(1):54-62, 2000 .

[226] M. T. Vanier, C. Rodriguez-Lafrasse, R. Rousson, N. Gazzah, M. C. Juge, P. G. Pentchev, A. Revol, and P. Louisot. Type c niemann-pick disease: Spectrum of phenotypic variation in disruption of intracellular ldl-derived cholesterol processing. Biochimica et Biophysica Acta - Molecular Basis of Disease, 1096(4):328-337, 1991.

[227] M. C. Gondré-Lewis, R. McGlynn, and S. U. Walkley. Cholesterol accumulation in npc1deficient neurons is ganglioside dependent. Current Biology, 13(15):1324-1329, 2003.

[228] F. M. Platt, G. Reinkensmeier, R. A. Dwek, and T. D. Butters. Extensive glycosphingolipid depletion in the liver and lymphoid organs of mice treated with n-butyldeoxynojirimycin. Journal of Biological Chemistry, 272(31):19365-19372, 1997. 
[229] M. Zervas, K. L. Somers, M. A. Thrall, and S. U. Walkley. Critical role for glycosphingolipids in niemann-pick disease type c. Current Biology, 11(16):1283-1287, 2001.

[230] Y. Liu, Y. P. Wu, R. Wada, E. B. Neufeld, K. A. Mullin, A. C. Howard, P. G. Pentchev, M. T. Vanier, K. Suzuki, and R. L. Proia. Alleviation of neuronal ganglioside storage does not improve the clinical course of the niemann-pick c disease mouse. Human molecular genetics, 9(7):1087-1092, 2000.

[231] B. Karten, D. E. Vance, R. B. Campenot, and J. E. Vance. Cholesterol accumulates in cell bodies, but is decreased in distal axons, of niemann-pick c1-deficient neurons. Journal of neurochemistry, 83(5):1154-1163, 2002.

[232] C. Xie, D. K. Burns, S. D. Turley, and J. M. Dietschy. Cholesterol is sequestered in the brains of mice with niemann-pick type $\mathrm{c}$ disease but turnover is increased. Journal of neuropathology and experimental neurology, 59(12):1106-1117, 2000.

[233] M. T. Vanier. Lipid changes in niemann-pick disease type c brain: Personal experience and review of the literature. Neurochemical research, 24(4):481-489, 1999.

[234] H. Weintraub, A. Abramovici, and U. Sandbank. Neurological mutation characterized by dysmyelination in nctr-balb/c mouse with lysosomal lipid storage disease. Journal of neurochemistry, 45(3):665-672, 1985.

[235] N. K. Dahl, K. L. Reed, M. A. Daunais, J. R. Faust, and L. Liscum. Isolation and characterization of chinese hamster ovary cells defective in the intracellular metabolism of low density lipoprotein-derived cholesterol. Journal of Biological Chemistry, 267(7):4889-4896, 1992.

[236] B. Karten, D. E. Vance, R. B. Campenot, and J. E. Vance. Trafficking of cholesterol from cell bodies to distal axons in niemann pick c1-deficient neurons. Journal of Biological Chemistry, 278(6):4168-4175, 2003.

[237] J. E. Vance. Lipid imbalance in the neurological disorder, niemann-pick c disease. FEBS letters, 580(23):5518-5524, 2006.

[238] A. Prasad, W. A. Fischer, R. A. Maue, and L. P. Henderson. Regional and developmental expression of the npc1 mrna in the mouse brain. Journal of neurochemistry, 75(3):1250-1257, 2000.

[239] W. Y. Ong, R. K. Sundaram, E. Huang, S. Ghoshal, U. Kumar, P. G. Pentchev, and S. C. Patel. Neuronal localization and association of niemann pick c2 protein (he1/npc2) with the postsynaptic density. Neuroscience, 128(3):561-570, 2004. 
[240] B. Karten, R. B. Campenot, D. E. Vance, and J. E. Vance. The niemann-pick c1 protein in recycling endosomes of presynaptic nerve terminals. Journal of Lipid Research, 47(3):504$514,2006$.

[241] T. C. Südhof. The synaptic vesicle cycle. Annual Reviews of Neuroscience, 27:509-547, 2004.

[242] P. A. March, M. A. Thrall, D. E. Brown, T. W. Mitchell, A. C. Lowenthal, and S. U. Walkley. Gabaergic neuroaxonal dystrophy and other cytopathological alterations in feline niemann-pick disease type c. Acta Neuropathologica, 94(2):164-172, 1997.

[243] S. U. Walkley and K. Suzuki. Consequences of npc1 and npc2 loss of function in mammalian neurons. Biochimica et Biophysica Acta - Molecular and Cell Biology of Lipids, 1685(1-3):4862,2004 .

[244] S. C. Patel, S. Suresh, U. Kumar, C. Y. Hu, A. Cooney, E. J. Blanchette-Mackie, E. B. Neufeld, R. C. Patel, R. O. Brady, Y. C. Patel, P. G. Pentchev, and W. Y. Ong. Localization of niemann-pick c1 protein in astrocytes: Implications for neuronal degeneration in niemannpick type c disease. Proceedings of the National Academy of Sciences of the United States of America, 96(4):1657-1662, 1999.

[245] D. C. German, E. Matthew Quintero, C.L-. Liang, B. Ng, S. Punia, C. Xie, and J. M. Dietschy. Selective neurodegeneration, without neurofibrillary tangles, in a mouse model of niemann-pick c disease. Journal of Comparative Neurology, 433(3):415-425, 2001.

[246] L. D. Griffin, W. Gong, L. Verot, and S. H. Mellon. Niemann-pick type c disease involves disrupted neurosteroidogenesis and responds to allopregnanolone. Nature medicine, 10(7):704$711,2004$.

[247] M. Zhang, N. K. Dwyer, D. C. Love, A. Cooney, M. Comly, E. Neufeld, P. G. Pentchev, E. J. Blanchette-Mackie, and J. A. Hanover. Cessation of rapid late endosomal tubulovesicular trafficking in niemann-pick type c1 disease. Proceedings of the National Academy of Sciences of the United States of America, 98(8):4466-4471, 2001.

[248] A. Choudhury, D. K. Sharma, D. L. Marks, and R. E. Pagano. Elevated endosomal cholesterol levels in niemann-pick cells inhibit rab4 and perturb membrane recycling. Molecular biology of the cell, 15(10):4500-4511, 2004.

[249] L. P. Henderson, L. Lin, A. Prasad, C. A. Paul, T. Y. Chang, and R. A. Maue. Embryonic striatal neurons from niemann-pick type c mice exhibit defects in cholesterol metabolism and neurotrophin responsiveness. Journal of Biological Chemistry, 275(26):20179-20187, 2000.

[250] J. Sambrook and D. Russell, editors. Molecular cloning: A laboratory manual. Cold Spring Harbor Laboratory Press, 3 edition, 2001. 
[251] C. Papworth, J. C. Bauer, J. Braman, and D. A. Wright. Quickchange site-directed mutagenesis. Strategies, 9:3-4, 1996.

[252] A. Lehninger, editor. Biochemistry. Worth publishes, Inc, 1975.

[253] H. Towbin, T. Staehelin, and J. Gordon. Electrophoretic transfer of proteins from polyacrylamide gels to nitrocellulose sheets: procedure and some applications. Proc Natl Acad Sci U $S$ A, 76(9):4350-4354, 1979.

[254] I. Fernow, A. Icking, and R. Tikkanen. Reggie-1 and reggie-2 localize in non-caveolar rafts in epithelial cells: Cellular localization is not dependent on the expression of caveolin proteins. European journal of cell biology, 86(6):345-352, 2007.

[255] T. Babuke and R. Tikkanen. Dissecting the molecular function of reggie/flotillin proteins. European journal of cell biology, 86(9):525-532, 2007.

[256] M. F. Langhorst, A. Reuter, F. A. Jaeger, F. M. Wippich, G. Luxenhofer, H. Plattner, and C. A. O. Stuermer. Trafficking of the microdomain scaffolding protein reggie-1/flotillin-2. European journal of cell biology, 87(4):211-226, 2008.

[257] J. D. Butler, J. Blanchette-Mackie, E. Goldin, R. R. O’Neill, G. Carstea, C. F. Roff, M. C. Patterson, S. Patel, M. E. Comly, A. Cooney, M. T. Vanier, R. O. Brady, and P. G. Pentchev. Progesterone blocks cholesterol translocation from lysosomes. Journal of Biological Chemistry, 267(33):23797-23805, 1992.

[258] K. Trajkovic, A. S. Dhaunchak, J. T. Goncalves, D. Wenzel, A. Schneider, G. Bunt, K. A. Nave, and M. Simons. Neuron to glia signaling triggers myelin membrane exocytosis from endosomal storage sites. Journal of Cell Biology, 172(6):937-948, 2006.

[259] C. H. V. Koh and N. S. Cheung. Cellular mechanism of u18666a-mediated apoptosis in cultured murine cortical neurons: Bridging niemann-pick disease type c and alzheimer's disease. Cellular signalling, 18(11):1844-1853, 2006.

[260] J. C. Cruz, S. Sugii, C. Yu, and T. Y. Chang. Role of niemann-pick type c1 protein in intracellular trafficking of low density lipoprotein-derived cholesterol. Journal of Biological Chemistry, 275(6):4013-4021, 2000.

[261] X. Hua, A. Nohturfft, J. L. Goldstein, and M. S. Brown. Sterol resistance in cho cells traced to point mutation in srebp cleavage-activating protein. Cell, 87(3):415-426, 1996.

[262] F. Bartz, L. Kern, D. Erz, M. Zhu, D. Gilbert, T. Meinhof, U. Wirkner, H. Erfle, M. Muckenthaler, R. Pepperkok, and H. Runz. Identification of cholesterol-regulating genes by targeted rnai screening. Cell Metabolism, 10(1):63-75, 2009. 
[263] B. U. Samuel, N. Mohandas, T. Harrison, H. McManus, W. Rosse, M. Reid, and K. Haldar. The role of cholesterol and glycosylphosphatidylinositol-anchored proteins of erythrocyte rafts in regulating raft protein content and malarial infection. Journal of Biological Chemistry, 276(31):29319-29329, 2001.

[264] C. C. Domingues, A. Ciana, A. Buttafava, B. R. Casadei, C. Balduini, E. De Paula, and G. Minetti. Effect of cholesterol depletion and temperature on the isolation of detergentresistant membranes from human erythrocytes. Journal of Membrane Biology, 234(3):195$205,2010$.

[265] A. Schneider, L. Rajendran, M. Honsho, M. Gralle, G. Donnert, F. Wouters, S. W. Hell, and M. Simons. Flotillin-dependent clustering of the amyloid precursor protein regulates its endocytosis and amyloidogenic processing in neurons. Journal of Neuroscience, 28(11):2874$2882,2008$.

[266] W. Möbius, E. van Donselaar, Y. Ohno-Iwashita, Y. Shimada, H. F. G. Heijnen, J. W. Slot, and H. J. Geuze. Recycling compartments and the internal vesicles of multivesicular bodies harbor most of the cholesterol found in the endocytic pathway. Traffic, 4(4):222-231, 2003.

[267] L. Rajendran, S. Le Lay, and H. Illges. Raft association and lipid droplet targeting of flotillins are independent of caveolin. Biological chemistry, 388(3):307-314, 2007.

[268] S. Kumari, S. Mg, and S. Mayor. Endocytosis unplugged: Multiple ways to enter the cell. Cell research, 20(3):256-275, 2010.

[269] M. Kirkham, S. J. Nixon, M. T. Howes, L. Abi-Rached, D. E. Wakeham, M. Hanzal-Bayer, C. Ferguson, M. M. Hill, M. Fernandez-Rojo, D. A. Brown, J. F. Hancock, F. M. Brodsky, and R. G. Parton. Evolutionary analysis and molecular dissection of caveola biogenesis. Journal of cell science, 121(12):2075-2086, 2008.

[270] K. Riento, M. Frick, I. Schafer, and B. J. Nichols. Endocytosis of flotillin-1 and flotillin-2 is regulated by fyn kinase. Journal of cell science, 122(7):912-918, 2009.

[271] I. Carcea, A. Ma'ayan, R. Mesias, B. Sepulveda, S. R. Salton, and D. L. Benson. Flotillinmediated endocytic events dictate cell type-specific responses to semaphorin 3a. Journal of Neuroscience, 30(45):15317-15329, 2010.

[272] S. Monier, R. G. Parton, F. Vogel, J. Behlke, A. Henske, and T. V. Kurzchalia. Vip21caveolin, a membrane protein constituent of the caveolar coat, oligomerizes in vivo and in vitro. Molecular biology of the cell, 6(7):911-927, 1995.

[273] E. Ikonen and R. G. Parton. Caveolins and cellular cholesterol balance. Traffic, 1(3):212-217, 2000 . 
[274] M. Murata, J. Peränen, R. Schreiner, F. Wieland, T. V. Kurzchalia, and K. Simons. Vip21/caveolin is a cholesterol-binding protein. Proceedings of the National Academy of Sciences of the United States of America, 92(22):10339-10343, 1995.

[275] E. J. Smart, Y. S. Ying, P. A. Conrad, and R. G. W. Anderson. Caveolin moves from caveolae to the golgi apparatus in response to cholesterol oxidation. Journal of Cell Biology, 127(5):1185-1197, 1994.

[276] A. Bist, P. E. Fielding, and C. J. Fielding. Two sterol regulatory element-like sequences mediate up-regulation of caveolin gene transcription in response to low density lipoprotein free cholesterol. Proceedings of the National Academy of Sciences of the United States of America, 94(20):10693-10698, 1997.

[277] P. E. Fielding. Plasma membrane caveolae mediate the efflux of cellular free cholesterol. Biochemistry, 34(44):14288-14292, 1995.

[278] C. J. Fielding, A. Bist, and P. E. Fielding. Intracellular cholesterol transport in synchronized human skin fibroblasts. Biochemistry, 38(8):2506-2513, 1999.

[279] P. E. Fielding and C. J. Fielding. Intracellular transport of low density lipoprotein derived free cholesterol begins at clathrin-coated pits and terminates at cell surface caveolae. Biochemistry, 35(47):14932-14938, 1996.

[280] P. G. Frank, M. W. Cheung, S. Pavlides, G. Llaverias, D. S. Park, and M. P. Lisanti. Caveolin1 and regulation of cellular cholesterol homeostasis. American Journal of Physiology - Heart and Circulatory Physiology, 291(2):H677-H686, 2006.

[281] D. K. Sharma, J. C. Brown, A. Choudhury, T. E. Peterson, E. Holicky, D. L. Marks, R. Simari, R. G. Parton, and R. E. Pagano. Selective stimulation of caveolar endocytosis by glycosphingolipids and cholesterol. Molecular biology of the cell, 15(7):3114-3122, 2004.

[282] W. S. Garver, R. P. Erickson, J. M. Wilson, T. L. Colton, G. S. Hossain, M. A. Kozloski, and R. A. Heidenreich. Altered expression of caveolin-1 and increased cholesterol in detergent insoluble membrane fractions from liver in mice with niemann-pick disease type c. Biochimica et Biophysica Acta - Molecular Basis of Disease, 1361(3):272-280, 1997.

[283] B. Brügger, B. Glass, P. Haberkant, I. Leibrecht, F. T. Wieland, and H. G. Kräusslich. The hiv lipidome: A raft with an unusual composition. Proceedings of the National Academy of Sciences of the United States of America, 103(8):2641-2646, 2006.

[284] M. Vidal, J. Sainte-Marie, J. R. Philippot, and A. Bienvenue. Asymmetric distribution of phospholipids in the membrane of vesicles relased during in vitro maturation of guinea pig 
reticulocytes: Evidence precluding a role for 'aminophospholipid translocase'. Journal of cellular physiology, 140(3):455-462, 1989.

[285] C. Lebrand, M. Corti, H. Goodson, P. Cosson, V. Cavalli, N. Mayran, J. Fauré, and J. Gruenberg. Late endosome motility depends on lipids via the small gtpase rab7. EMBO Journal, 21(6):1289-1300, 2002.

[286] E. O. Freed and Martin M. A. HIVs and their replication. Lippincott Williams and Wilkoins Philadelphia, 2 edition, 2001.

[287] P. Leblanc, S. Alais, I. Porto-Carreiro, S. Lehmann, J. Grassi, G. Raposo, and J. L. Darlix. Retrovirus infection strongly enhances scrapie infectivity release in cell culture. EMBO Journal, 25(12):2674-2685, 2006.

[288] E. O. Freed. Hiv-1 gag proteins: Diverse functions in the virus life cycle. Virology, 251(1):1$15,1998$.

[289] M. Ikeda and R. Longnecker. Cholesterol is critical for epstein-barr virus latent membrane protein 2a trafficking and protein stability. Virology, 360(2):461-468, 2007.

[290] L. Liscum. Niemann-pick type c mutations cause lipid traffic jam. Traffic, 1(3):218-225, 2000.

[291] L. Aguilar, G. Ortega-Pierres, B. Campos, R. Fonseca, M. Ibáñez, C. Wong, N. Farfán, J. M. Naciff, M. A. Kaetzel, J. R. Dedman, and I. Baeza. Phospholipid membranes form specific nonbilayer molecular arrangements that are antigenic. Journal of Biological Chemistry, 274(36), 1999.

[292] R. C. Sexton, S. R. Panini, F. Azran, and H. Rudney. Effects of 3beta-[2(diethylamino)ethoxy]androst-5-en-17-one on the synthesis of cholesterol and ubiquinone in rat intestinal epithelial cell cultures. Biochemistry, 22(25):5687-5692, 1983.

[293] S. E. Phillips, E. A. Woodruff III, P. Liang, M. Patten, and K. Broadie. Neuronal loss of drosophila npcla causes cholesterol aggregation and age-progressive neurodegeneration. Journal of Neuroscience, 28(26):6569-6582, 2008.

[294] G. Liao, Y. Yao, J. Liu, Z. Yu, S. Cheung, A. Xie, X. Liang, and X. Bi. Cholesterol accumulation is associated with lysosomal dysfunction and autophagic stress in npc1-/- mouse brain. American Journal of Pathology, 171(3):962-975, 2007.

[295] K. Von Figura, V. Gieselmann, and J. Jaeken. Metachromatic leukodystrophy. The Metabolic and Molecular Bases of Inherited Disease, pages 3695-3724, 2001. 
[296] H. Ramakrishnan, K. K. Hedayati, R. Lüllmann-Rauch, C. Wessig, S. N. Fewou, H. Maier, H. H. Goebel, V. Gieselmann, and M. Eckhardt. Increasing sulfatide synthesis in myelinforming cells of arylsulfatase a-deficient mice causes demyelination and neurological symptoms reminiscent of human metachromatic leukodystrophy. Journal of Neuroscience, 27(35):9482-9490, 2007.

[297] D. Klein, H. Büssow, S. N. Fewou, and V. Gieselmann. Exocytosis of storage material in a lysosomal disorder. Biochemical and biophysical research communications, 327(3):663-667, 2005.

[298] D. J. Selkoe. Alzheimer's disease results from the cerebral accumulation and cytotoxicity of amyloid beta-protein. Journal of Alzheimer's Disease, 3(1):75-81, 2001.

[299] F. M. LaFerla, K. N. Green, and S. Oddo. Intracellular amyloid-beta in alzheimer's disease. Nature Reviews Neuroscience, 8(7):499-509, 2007.

[300] D. Z. Christensen, T. Schneider-Axmann, P. J. Lucassen, T. A. Bayer, and O. Wirths. Accumulation of intraneuronal abeta correlates with apoe4 genotype. Acta Neuropathologica, 119(5):555-566, 2010.

[301] D. Z. Christensen, T. A. Bayer, and O. Wirths. Intracellular abeta triggers neuron loss in the cholinergic system of the app/ps1ki mouse model of alzheimer's disease. Neurobiology of aging, 31(7):1153-1163, 2010.

[302] E. Emmanouilidou, K. Melachroinou, T. Roumeliotis, S. D. Garbis, M. Ntzouni, L. H. Margaritis, L. Stefanis, and K. Vekrellis. Cell-produced alpha-synuclein is secreted in a calciumdependent manner by exosomes and impacts neuronal survival. Journal of Neuroscience, 30(20):6838-6851, 2010.

[303] K. Lauber, S. G. Blumenthal, M. Waibel, and S. Wesselborg. Clearance of apoptotic cells: Getting rid of the corpses. Molecular cell, 14(3):277-287, 2004.

[304] P. Bette-Bobilo and M. Vidal. Characterization of phospholipase a2 activity in reticulocyte endocytic vesicles. European Journal of Biochemistry, 228(1):199-205, 1995.

[305] S. J. Kim, D. Gershov, X. Ma, N. Brot, and K. B. Elkon. I-pla2 activation during apoptosis promotes the exposure of membrane lysophosphatidylcholine leading to binding by natural immunoglobulin m antibodies and complement activation. Journal of Experimental Medicine, 196(5):655-665, 2002.

[306] K. Lauber, E. Bohn, S. M. Kröber, Y. J. Xiao, S. G. Blumenthal, R. K. Lindemann, P. Marini, C. Wiedig, A. Zobywalski, S. Baksh, Y. Xu, I. B. Autenrieth, K. Schulze-Osthoff, C. Belka, G. Stuhler, and S. Wesselborg. Apoptotic cells induce migration of phagocytes via caspase3-mediated release of a lipid attraction signal. Cell, 113(6):717-730, 2003. 
[307] L. Zakharova, M. Svetlova, and A. F. Fomina. T cell exosomes induce cholesterol accumulation in human monocytes via phosphatidylserine receptor. Journal of cellular physiology, 212(1):174-181, 2007.

[308] C. K. Glass and J. L. Witztum. Atherosclerosis: The road ahead. Cell, 104(4):503-516, 2001.

[309] M. S. Brown and J. L. Goldstein. Lipoprotein metabolism in the macrophage: Implications for cholesterol deposition in atherosclerosis. Annual Review of Biochemistry, Vol. 52:223-261, 1983.

[310] J. R. Zhang, T. Coleman, S. J. Langmade, D. E. Scherrer, L. Lane, M. H. Lanier, C. Feng, M. S. Sands, J. E. Schaffer, C. F. Semenkovich, and D. S. Ory. Niemann-pick c1 protects against atherosclerosis in mice via regulation of macrophage intracellular cholesterol trafficking. Journal of Clinical Investigation, 118(6):2281-2290, 2008.

[311] G. Rabinowits, C. Gerçel-Taylor, J. M. Day, D. D. Taylor, and G. H. Kloecker. Exosomal microrna: A diagnostic marker for lung cancer. Clinical Lung Cancer, 10(1):42-46, 2009.

[312] T. Pisitkun, R. Johnstone, and M. A. Knepper. Discovery of urinary biomarkers. Molecular and Cellular Proteomics, 5(10):1760-1771, 2006.

[313] M. T. Vanier. Niemann-pick disease type c. Orphanet Journal of Rare Diseases, 5(1), 2010.

[314] J. E. Wraith, M. R. Baumgartner, B. Bembi, A. Covanis, T. Levade, E. Mengel, M. Pineda, F. Sedel, M. Topçu, M. T. Vanier, H. Widner, F. A. Wijburg, and M. C. Patterson. Recommendations on the diagnosis and management of niemann-pick disease type c. Molecular genetics and metabolism, 98(1-2):152-165, 2009.

[315] C. Iturriaga, M. Pineda, E. M. Fernández-Valero, M. T. Vanier, and M. J. Coll. Niemannpick c disease in spain: Clinical spectrum and development of a disability scale. Journal of the neurological sciences, 249(1):1-6, 2006.

[316] N. M. Yanjanin, J. I. Velez, A. Gropman, K. King, S. E. Bianconi, S. K. Conley, C. C. Brewer, B. Solomon, W. J. Pavan, M. Arcos-Burgos, M. C. Patterson, and F. D. Porter. Linear clinical progression, independent of age of onset, in niemann-pick disease, type c. American Journal of Medical Genetics, Part B: Neuropsychiatric Genetics, 153B:132-140, 2009.

[317] A. Michael, S. D. Bajracharya, P. S. T. Yuen, H. Zhou, R. A. Star, G. G. Illei, and I. Alevizos. Exosomes from human saliva as a source of microrna biomarkers. Oral diseases, 16(1):34-38, 2010. 
[318] I. Dimov, L. J. Velickovic, and V. Stefanovic. Urinary exosomes. The Scientific World Journal, 9:1107-1118, 2009.

[319] H. Sonoda, N. Yokota-Ikeda, S. Oshikawa, Y. Kanno, K. Yoshinaga, K. Uchida, Y. Ueda, K. Kimiya, S. Uezono, A. Ueda, K. Ito, and M. Ikeda. Decreased abundance of urinary exosomal aquaporin-1 in renal ischemia-reperfusion injury. American Journal of Physiology - Renal Physiology, 297(4):F1006-F1016, 2009.

[320] J. L. Welton, S. Khanna, P. J. Giles, P. Brennan, I. A. Brewis, J. Staffurth, M. D. Mason, and A. Clayton. Proteomics analysis of bladder cancer exosomes. Molecular and Cellular Proteomics, 9(6):1324-1338, 2010.

[321] E. J. Hoorn, T. Pisitkun, R. Zietse, P. Gross, J. Frokiaer, N. S. Wang, P. A. Gonzales, R. A. Star, and M. A. Knepper. Prospects for urinary proteomics: Exosomes as a source of urinary biomarkers. Nephrology, 10(3):283-290, 2005.

[322] P. V. Bottini, M. A. Ribeiro Alves, and C. R. Garlipp. Electrophoretic pattern of concentrated urine: comparison between 24-hour collection and random samples. American Journal of Kidney Diseases : The Official Journal of the National Kidney Foundation, 39(1):E2, 2002.

[323] M. Shinada, A. Akdeniz, S. Panagiotopoulos, G. Jerums, and L. A. Bach. Proteolysis of insulin-like growth factor-binding protein-3 is increased in urine from patients with diabetic nephropathy. Journal of Clinical Endocrinology and Metabolism, 85(3):1163-1169, 2000.

[324] H. Zhou, P. S. T. Yuen, T. Pisitkun, P. A. Gonzales, H. Yasuda, J. W. Dear, P. Gross, M. A. Knepper, and R. A. Star. Collection, storage, preservation, and normalization of human urinary exosomes for biomarker discovery. Kidney international, 69(8):1471-1476, 2006.

[325] S. Kennedy. Proteomic profiling from human samples: The body fluid alternative. Toxicology letters, 120(1-3):379-384, 2001. 


\title{
Curriculum Vitae
}

\author{
Katrin Strauss \\ born on October $15^{\text {th }} 1983$ in Sondershausen, Germany \\ Hermann-Rein-Str. 3, 37075, Göttingen, Germany \\ Phone: +49 (0) 5513899534 \\ E-Mail: strauss@em.mpg.de
}

\section{Education and Research Experience}

2007 - present Max Planck Institute of Experimental Medicine, Göttingen, Germany: Doctoral thesis 'Exosomes act as molecular vehicles contributing to cellular cholesterol efflux'

2007 - present Georg-August-University, Göttingen, Germany: Ph.D. program 'Molecular Medicine'

2002 - 2007 Martin-Luther-University, Halle (Saale), Germany: Institute for Biology, Diploma degree in biology

2005

University of Pretoria, Pretoria, South Africa: Department of Zoology and Entomology: research semester

1994 - 2002 Geschwister-Scholl-Gymnasium, Sondershausen, Germany: Abitur degree (general qualification for university entrance)

\section{Awards and Scholarships}

2005

Scholarship of DAAD (German Academic Exchange Service) for research semester at the University of Pretoria, South Africa

2004

E-fellows scholarship for best intermediate diploma ('Vordiplom') in biology at the Martin-Luther-University Halle (Saale) in 2004

2002

Certificate for best high school graduation at the Geschwister-SchollGymnasium Sondershausen in 2002 


\section{Publications}

Strauss K., Goebel C., Runz H., Möbius W., Weiss S., Feussner I., Simons M., and Schneider A. (2010) Exosome Secretion Ameliorates Lysosomal Storage of Cholesterol in Niemann-Pick Type C Disease. The Journal of Biological Chemistry; 285(34): 26279-26288

Strauss K., Scharpenberg H., Crewe R.M., Glahn F., Foth H., and Moritz R.F.A. (2008) The role of the queen mandibular gland pheromone in honeybees (Apis mellifera): honest signal or suppressive agent? Behavioral Ecology and Sociobiology; 62(9): 1523-1531

Human H., Nicolson S.W., Strauss K., Pirk C.W.W., and Dietemann V. (2007) Influence of pollen quality on ovarian development in honeybee workers (Apis mellifera scutellata); Journal of Insect Physiology 53(7): 649-655 